TRANSACTIONS OF THE

AMERICAN MATHEMATICAL SOCIETY

Volume 356, Number 2, Pages 757-803

S 0002-9947(03)03318-X

Article electronically published on August 21, 2003

\title{
DERIVING CALCULUS WITH COTRIPLES
}

\author{
B. JOHNSON AND R. MCCARTHY
}

\begin{abstract}
We construct a Taylor tower for functors from pointed categories to abelian categories via cotriples associated to cross effect functors. The tower was inspired by Goodwillie's Taylor tower for functors of spaces, and is related to Dold and Puppe's stable derived functors and Mac Lane's $Q$-construction. We study the layers, $D_{n} F=\operatorname{fiber}\left(P_{n} F \rightarrow P_{n-1} F\right)$, and the limit of the tower. For the latter we determine a condition on the cross effects that guarantees convergence. We define differentials for functors, and establish chain and product rules for them. We conclude by studying exponential functors in this setting and describing their Taylor towers.
\end{abstract}

The calculus of homotopy functors, developed by Tom Goodwillie, is an increasingly important tool in homotopy theory and algebraic topology. For a functor of spaces $F$ and a space $X$ satisfying certain connectivity conditions, Goodwillie (G3]) has shown that there is a tower of functors and natural transformations,

$$
\begin{aligned}
& \vdots \\
& \downarrow \\
& P_{n+1} F_{X} \\
& p_{n+1} F_{X} \nearrow \quad \downarrow q_{n+1} F_{X} \\
& F_{X} \stackrel{p_{n} F_{X}}{\longrightarrow} \quad P_{n} F_{X} \\
& p_{n-1} F_{X} \searrow \quad \downarrow q_{n} F_{X} \\
& P_{n-1} F_{X} \\
& \vdots \\
& \downarrow \\
& P_{0} F_{X}=F(X)
\end{aligned}
$$

that acts like a Taylor series for the functor $F$ expanded about the space $X$. That is, each $P_{n} F_{X}$ can be likened to a degree $n$ approximation of $F$, and the "Taylor tower" above converges to $F$ in a range of spaces that depends on $F$ and $X$. The Taylor tower has been used by Greg Arone and Mark Mahowald ( $\mathrm{A}-\mathrm{M}])$ in their

Received by the editors January 20, 1999 and, in revised form, February 18, 2003.

2000 Mathematics Subject Classification. Primary 18G30; Secondary 55P65, 55U15.

The second author was supported by National Science Foundation grant \# 1-5-30943 and a Sloan Fellowship. 
work on the $v_{n}$-periodic homotopy of odd spheres. Using "orthogonal calculus", a version of calculus developed by Michael Weiss ([W]), Arone ([A] ) has also shown that the Mitchell-Richter filtration of loops on Stiefel manifolds stably splits.

The linear piece of Goodwillie's tower or "differential",

$$
D_{1} F_{X}=\operatorname{fiber}\left(P_{1} F_{X}(-) \rightarrow F(X)\right),
$$

is often enough to determine a functor up to a constant, in that a natural transformation $\eta: F \rightarrow G$ that induces an equivalence $D_{1} \eta_{X}: D_{1} F_{X} \rightarrow D_{1} G_{X}$ for all spaces $X$ yields an equivalence

$$
\text { fiber }(F(Y) \rightarrow F(X)) \rightarrow \operatorname{fiber}(G(Y) \rightarrow G(X)),
$$

when $F, G, X$, and the space $Y$ satisfy some connectivity conditions ([G2]). This latter result has been instrumental in establishing strong connections between algebraic $K$-theory and more computable theories ( $\mathrm{CCGH}$, [M1).

Goodwillie's existence proof for the tower is constructive, but provides one with a model that is difficult to work with in general. For $n=1$, the construction is reminiscent of the stabilization of functors of additive categories defined by DoldPuppe ([D-P]), and Eilenberg-Mac Lane ([E-M1], [E-M2]). We explored this similarity in [J-M1], and established conditions under which Goodwillie's degree 1 approximations were the same as Dold-Puppe's stabilization and Mac Lane's Qconstruction. The work of Dold-Puppe and Eilenberg-Mac Lane suggested a simpler model for the Taylor tower in the algebraic setting. A generalization of Mac Lane's $Q$-construction was used in [J-M2] to produce such a model.

In the first part of this paper we produce a more natural and simpler means for constructing a Taylor tower for functors from a basepointed category (a category with an object that is both initial and final) to a category of chain complexes. The idea behind this construction is as follows. The failure of a functor $F$ to be "degree $n$ " is captured by the $n+1$ st cross effect functor, $c r_{n+1} F$, as defined by Eilenberg and Mac Lane. To construct a degree $n$ approximation to $F$, one seeks to "kill off" the $n+1$ st cross effect of $F$ by making a chain complex with $F$ in degree 0 and $c r_{n+1} F$ in degree 1 and taking the homology of this chain complex. This produces a right exact, strictly degree $n$ functor. However, when working homologically one would like to have a degree $n$ functor that is exact, or at least quasi-exact (takes short exact sequences to sequences that produce long exact sequences in homology). One solution to this problem is to take a resolution of the functor before killing off the cross effect. This approach is used by Teimuraz Pirashvili in $\left[\mathrm{P}\right.$. Another solution to this problem is to use the mapping cone of $\mathrm{cr}_{n+1} F \stackrel{+}{\longrightarrow} F$. This produces a quasi-exact functor, but the degree 2 homology of this functor, $\operatorname{ker}\left(c r_{n+1} F \stackrel{+}{\longrightarrow} F\right)$, is no longer degree $n$. So, we try again to make this new construction degree $n$ by killing off the $n+1$ st cross effect of $\operatorname{ker}\left(c r_{n+1} F \stackrel{+}{\longrightarrow} F\right)$, and introducing a three-stage chain complex where the degree 2 differential factors through the kernel of + ,

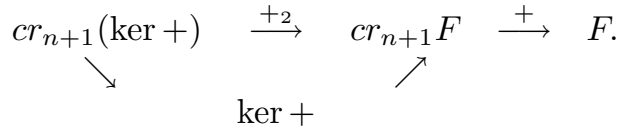

But, one faces problems similar to the ones described above, in that $\operatorname{ker}\left(+_{2}\right)$ is not degree $n$. One can try to fix the problem in a similar fashion by placing the cross effect of the kernel in degree 3. Continuing in this fashion produces a sequence 
of chain complexes in which we are repeatedly taking cross effects, and the failure to be degree $n$ is pushed into higher and higher homological degree. Taking the limit of this process produces a chain complex of functors that is degree $n$ and quasi-exact.

By utilizing a cotriple associated to the $n+1$ st cross effect functor, we obtain such a chain complex as an immediate consequence of standard cotriple properties. Moreover, all of the essential features of a Taylor tower are also easily derived from basic cotriple properties applied to this setting. The cotriple model for the Taylor tower can be readily dualized, and the dual version of calculus is presented in M2. The cotriple construction for a Taylor tower developed here has also been modified to produce a new tower for a functor of spaces in [MO]. This new tower tends to agree with Goodwillie's Taylor tower for spaces in the functor's range of analyticity. In J-M3 we will show how the cotriple model can be used to classify degree $n$ functors to chain complexes in terms of modules over certain differential graded algebras.

In addition to showing how to construct Taylor towers for functors from basepointed to abelian categories, we investigate several important functors related to these towers. We first study the layers, $D_{n} F=\operatorname{fiber}\left(P_{n} F \rightarrow P_{n-1} F\right)$, of the Taylor tower, and show that they can be obtained directly from the $n$th cross effect of $F$. In particular, we prove

Proposition 3.9. For any functor $F: \mathcal{C} \rightarrow \mathcal{A}$, the functor $D_{n} F$ is naturally equivalent to $D_{1}^{(n)} \mathrm{cr}_{n} F_{h \Sigma_{n}}$.

This result is analogous to a result of Goodwillie's for the layers in the Taylor tower of a functor of spaces. It is useful in determining the layers of a tower, and will be used in the last section to compute a version of the Taylor tower for exponential functors.

We then examine the limit of the Taylor tower, and determine a connectivity condition on the cross effects of $F$ that guarantees that the limit of the Taylor tower agrees with $F$. We study the behavior of the degree $n$ approximations to the functor $F$ arising from the Taylor tower in the direction of an object $X$. That is, for a fixed object $X$ and functor $F$, we consider the functor

$$
F_{X}(C)=\operatorname{kernel}[F(C \vee X) \rightarrow F(X)] .
$$

We prove a continuation principle in terms of such functors:

Proposition 4.14. Let $\eta: F \longrightarrow G$ be a natural transformation of functors from $\mathcal{C}$ to $C h \mathcal{A}$. The following are equivalent.

a) $P_{1} \eta_{X}$ is an equivalence for all $X \in \mathcal{C}$.

b) $P_{n} \eta_{X}$ is an equivalence for all $n$ and all $X \in \mathcal{C}$.

c) $P_{\infty} \eta_{X}$ is an equivalence for all $X \in \mathcal{C}$ (where $P_{\infty} \eta_{X}$ denotes the limit of the $P_{n} \eta_{X}$ 's).

When applied to functors whose Taylor towers converge, this result tells us that the functor $F$ is determined by its linear part up to the constant $F(X)$. We also develop the notion of differential for a functor $F$ in the direction of an object $X, \nabla F(X, Y)$, by means of $F_{X}$. We prove a chain rule and product rule for the differentials and determine the behavior of the differential with respect to the other Taylor tower constructions. The chain rule is as follows. 
Proposition 5.6. Let $\mathcal{C}$ be a basepointed category with finite coproducts and let $\mathcal{A}$ and $\mathcal{A}^{\prime}$ be cocomplete abelian categories. Let $G: \mathcal{C} \longrightarrow \mathcal{A}^{\prime}$ and $F: \mathcal{A}^{\prime} \longrightarrow C h \mathcal{A}$ be functors. There is a natural isomorphism of bifunctors

$$
\nabla(F \circ G)(X, Y) \cong \nabla F(\nabla G(X, Y), G(Y)) .
$$

In the last section of the paper, we use the techniques and ideas of the previous sections to determine the functors that play the role of exponential functors for our theory. We will show that any exponential functor $F$ from a pointed category $\mathcal{C}$ to the category of modules over a commutative ring $\mathbb{K}$ factors as $\operatorname{Exp} \circ A$, where Exp is the forgetful functor from commutative differential graded $\mathbb{K}$-algebras to chain complexes of $\mathbb{K}$-modules and $A$ is a coproduct-preserving functor. We also compute a modified version of the Taylor tower (one in which the functors preserve quasi-isomorphisms of DG $\mathbb{K}$-algebras) for Exp and show that the $n$th term in this tower can be considered rationally as the derived functor of $I / I^{n}$ (in model category terms). We then indicate how one can extend this integrally using $E_{\infty}-\mathbb{K}$-algebras.

Although the work in this paper was inspired by the topological results of Goodwillie and the work of Dold-Puppe and Eilenberg-Mac Lane, none of these works should be considered prerequisites for this paper. Our treatment of the Taylor tower defined herein is self-contained and requires only some basic homological algebra and category theory.

The paper is organized as follows. In section 1 we review cross effects and the adjoint pairs needed for our cotriple construction. In section 2 we construct the Taylor tower and establish its basic properties. In section 3 we consider the layers, $D_{n} F=\operatorname{fiber}\left(P_{n} F \rightarrow P_{n-1} F\right)$, of the Taylor tower and show that they can be obtained directly from the $n$th cross effect of $F$. In section 4 we establish the convergence conditions for Taylor towers together with the continuation theorem. In section 5, we define the differential of a functor and prove the chain rule and product rule for these differentials, while also studying their relationship to other functors associated with the Taylor tower. In section 6 , we identify the exponential functors from pointed categories to modules over a commutative ring and study Taylor towers for such functors.

\section{Cross effects}

The key component of our universal degree $n$ tower construction is the $n$th cross effect of a functor. We devote this section to reviewing several properties of cross effects. We will also discuss two pairs of adjoint functors involving cross effects that can be used to construct degree $n$ towers for $F$.

Classically, cross effects were defined to study the degree of analytic functions of real variables. In particular, the $(n+1)$ st cross effect of a function $f$ is a function of $n+1$ variables that measures the extent to which $f$ fails to be degree $n$. For example, a function $f$ such that $f(0)=0$ is linear if and only if $f(x+y)=f(x)+f(y)$ for all $x$ and $y$. Moreover, this condition implies that $f(x)=f(1) \cdot x$ for all $x$. If $f$ is not linear and $f(0)=0$, then the failure of $f$ to be linear is captured by the second cross effect functor, $\mathrm{cr}_{2} f: \mathbf{R}^{2} \rightarrow \mathbf{R}$, defined by

$$
c r_{2} f(x, y)=f(x+y)-f(x)-f(y) .
$$

Furthermore, $f$ is degree 2 if and only if $c r_{2} f$ is linear in each variable. In this case, $c r_{2} f(x, y)=c r_{2} f(1,1)(x \cdot y)$ and $f(x)=f(1) \cdot x+c r_{2} f(1,1) \cdot\left(\begin{array}{l}x \\ 2\end{array}\right)$, where $\left(\begin{array}{l}x \\ 2\end{array}\right)=\frac{x(x-1)}{2}$ is the Vandermonde function. One can extend this definition inductively and let 
the $n$th cross effect, $c r_{n} f: \mathbf{R}^{\times n} \rightarrow \mathbf{R}$, be defined by setting $c r_{n} f\left(x_{1}, \ldots, x_{n}\right)$ equal to

$$
c r_{n-1} f\left(x_{1}+x_{2}, x_{3}, \ldots, x_{n}\right)-c r_{n-1} f\left(x_{1}, x_{3}, \ldots, x_{n}\right)-c r_{n-1} f\left(x_{2}, x_{3}, \ldots, x_{n}\right) .
$$

Cross effects are also defined when $f(0) \neq 0$. For such functions, we set $\operatorname{cr}_{1} f(x)=$ $f(x)-f(0)$, and define higher cross effects inductively as above. The cross effects have the following properties:

1) $c r_{n} f$ is symmetric with respect to its $n$ variables.

2) If $x_{i}=0$ for any $1 \leq i \leq n$, then $c r_{n} f\left(x_{1}, \ldots, x_{n}\right)=0$.

3) $f\left(x_{1}+\cdots+x_{n}\right)=f(0)+\sum_{\left\{s_{1}, \ldots, s_{k}\right\} \subseteq\{1, \ldots, n\}} c r_{k} f\left(x_{s_{1}}, \ldots, x_{s_{k}}\right)$.

Cross effects measure the degree of $f$ in that $c r_{n+1} f=0$ if and only if $f$ is degree $n$. In this case

$$
f(x)=f(0)+c r_{1} f(1) \cdot x+c r_{2} f(1,1)\left(\begin{array}{l}
x \\
2
\end{array}\right)+\cdots+c r_{n} f(1, \ldots, 1)\left(\begin{array}{l}
x \\
n
\end{array}\right),
$$

where $\left(\begin{array}{l}x \\ n\end{array}\right)=\frac{x(x-1) \cdots(x-(n-1))}{n !}$.

The notion of cross effect was extended to functors of additive categories by Eilenberg and Mac Lane. We summarize this extension below. We let $\mathcal{C}$ be a basepointed category with finite coproducts (denoted by $\vee$ ), and we let $\mathcal{A}$ be an abelian category.

Definition 1.1 ([E-M2]). Let $F: \mathcal{C} \rightarrow \mathcal{A}$. The $n$th cross effect of $F$ is the functor $c r_{n} F: \mathcal{C}^{\times n} \rightarrow \mathcal{A}$ defined inductively by

$$
\begin{gathered}
c r_{1} F(M) \oplus F(*) \cong F(M), \\
c r_{2} F\left(M_{1}, M_{2}\right) \oplus c r_{1} F\left(M_{1}\right) \oplus c r_{1} F\left(M_{2}\right) \cong c r_{1} F\left(M_{1} \vee M_{2}\right),
\end{gathered}
$$

and, in general,

$$
\begin{aligned}
& c r_{n} F\left(M_{1}, \ldots, M_{n}\right) \oplus c r_{n-1} F\left(M_{1}, M_{3}, \ldots, M_{n}\right) \oplus c r_{n-1} F\left(M_{2}, M_{3}, \ldots, M_{n}\right) \\
& \quad \cong c r_{n-1} F\left(M_{1} \vee M_{2}, M_{3}, \ldots, M_{n}\right) .
\end{aligned}
$$

As was the case with functions of real numbers, $c r_{n} F$ has the following properties.

Proposition 1.2. Let $F: \mathcal{C} \rightarrow \mathcal{A}$, and let $M_{1}, M_{2}, \ldots, M_{n}, M$ be objects in $\mathcal{C}$.

1) $c r_{n} F$ is symmetric with respect to its $n$ variables, i.e., for every $\sigma \in \Sigma_{n}$, the $n$th symmetric group, $c r_{n} F\left(M_{1}, \ldots, M_{n}\right) \cong c r_{n} F\left(M_{\sigma(1)}, \ldots, M_{\sigma(n)}\right)$.

2) $c r_{n} F\left(M_{1}, \ldots, M_{n}\right) \cong 0$ if any $M_{i}=*$.

3)

$$
F\left(M_{1} \vee \cdots \vee M_{n}\right) \cong F(*) \oplus\left(\bigoplus_{p=1}^{n}\left(\bigoplus_{j_{1}<\ldots<j_{p}} c r_{p} F\left(M_{j_{1}}, \ldots, M_{j_{p}}\right)\right)\right)
$$

and

$$
F\left(\bigvee_{i=1}^{n} M\right) \cong F(*) \oplus \bigoplus_{p=1}^{n}\left(\begin{array}{l}
n \\
p
\end{array}\right) c r_{p} F(M)
$$

where $\mathrm{cr}_{p} F(M)$ denotes $\mathrm{cr}_{p} F(M, \ldots, M)$.

Motivated by the relationship between degree and cross effects for functions of real variables, we use cross effects to define the degree of a functor. 
Definition 1.3. A functor $F$ from $\mathcal{C}$ to $\mathcal{A}$ is degree $n$ if $c r_{n+1} F \cong 0$.

Example 1.4. 1) Let $R$ be a ring, ${ }_{R} M_{R}$ the category of $R$-R-bimodules, and $T^{n}:{ }_{R} M_{R} \rightarrow{ }_{R} M_{R}$ the functor that takes a module to its $n$-fold tensor product over $R$. For $n=2$ and bimodules $M_{1}$ and $M_{2}$ we have

$$
\begin{aligned}
& T^{2}\left(M_{1} \oplus M_{2}\right) \\
& \quad \cong\left(M_{1} \otimes M_{1}\right) \oplus\left(M_{2} \otimes M_{2}\right) \oplus\left(M_{1} \otimes M_{2}\right) \oplus\left(M_{2} \otimes M_{1}\right) \\
& \quad \cong T^{2}\left(M_{1}\right) \oplus T^{2}\left(M_{2}\right) \oplus\left(M_{1} \otimes M_{2}\right) \oplus\left(M_{2} \otimes M_{1}\right) .
\end{aligned}
$$

Hence,

$$
c r_{2} T^{2}\left(M_{1}, M_{2}\right) \cong\left(M_{1} \otimes M_{2}\right) \oplus\left(M_{2} \otimes M_{1}\right) .
$$

In general,

$$
\begin{aligned}
c r_{k} T^{n}\left(M_{1}, \ldots, M_{n}\right) & \cong \bigoplus_{\alpha \in \operatorname{Epi}(\{1,2, \ldots, n\},\{1,2, \ldots, k\})} M_{\alpha(1)} \otimes \cdots \otimes M_{\alpha(n)},
\end{aligned}
$$

where $\operatorname{Epi}(\{1,2, \ldots, n\},\{1,2, \ldots, k\})$ is the set of surjections from the set $\{1,2$, $\ldots, n\}$ to the set $\{1,2, \ldots, k\}$. Note that this implies that $c r_{k} T^{n} \cong 0$ for $k>n$, so that $T^{n}$ is degree $n$. Similarly, if $R$ is commutative and $F^{n}$ is the $n$-fold symmetric power or exterior power functor, one can show that

$$
\begin{aligned}
& c r_{k} F^{n}\left(M_{1}, \ldots, M_{k}\right) \\
& \quad \cong \bigoplus_{\substack{t_{1}+\cdots+t_{k}=n \\
t_{1}, \ldots, t_{k}>0}} F^{t_{1}}\left(M_{1}\right) \otimes \cdots \otimes F^{t_{k}}\left(M_{k}\right),
\end{aligned}
$$

by again using a direct sum decomposition of $F^{n}\left(M_{1} \oplus M_{2} \oplus \cdots \oplus M_{k}\right)$ and noting that terms not involving all of the $M_{i}$ 's are, for some $j$, direct summands of $F^{n}\left(M_{1} \oplus \cdots \oplus \widehat{M}_{j} \oplus \cdots \oplus M_{k}\right)$, and as a consequence, belong to lower order cross effects. Since $c r_{k} F^{n} \cong 0$ for $k>n$, the $n$th symmetric and exterior power functors are degree $n$ as well.

2) Let $\mathcal{T}_{*}$ be the category of compactly generated topological spaces with nondegenerate basepoints, and let $\mathrm{Ab}$ be the category of abelian groups. For $n \geq 0$, let $H_{n}: \mathcal{T}_{*} \rightarrow \mathrm{Ab}$ be the functor that takes a space $X$ to its $n$th singular homology group, $H_{n}(X)$. Since $H_{n}(X \vee Y) \cong H_{n}(X) \oplus H_{n}(Y)$ for spaces $X$ and $Y$, it follows that $c r_{k} H_{n} \cong 0$ for $k \geq 2$ and $H_{n}$ is degree 1 . On the other hand, for $n>1$, the functor $\pi_{n}: \mathcal{T}_{*} \rightarrow$ Ab that takes $X$ to its $n$th homotopy group does not have finite degree, since the Hilton-Milnor theorem guarantees that $\pi_{n}$ has no non-vanishing cross effects.

3) Let Comm $_{\mathbb{K}}$ be the category of non-unital commutative differential graded $\mathbb{K}$-algebras over a commutative ring $\mathbb{K}, C h(\mathcal{K})$ the category of chain complexes of $\mathbb{K}$-modules, and Exp : $\operatorname{Comm}_{\mathbb{K}} \rightarrow C h(\mathcal{K})$ the forgetful functor. Since $X \vee Y=$ $X \oplus Y \oplus(X \otimes Y)$ for $X, Y \in \mathrm{Comm}_{\mathbb{K}}$, it follows that $\mathrm{cr}_{2} \operatorname{Exp}(X, Y) \cong X \otimes Y$. We will see in section 6 that $\operatorname{Exp}$ does not have finite degree.

Remark 1.5. The $n$th cross effect can also be defined as the homology of the total complex of a particular $n$-complex of objects in $\mathcal{A}$. Let $\mathcal{P}(\langle n\rangle)$ denote the power set of $\langle n\rangle=\{1,2, \ldots, n\}$ and let $C_{1}, \ldots, C_{n}$ be objects in $\mathcal{C}$. Let $\bar{C}$ be the $n$-cubical 
object in $\mathcal{C}$ with $\bar{C}(U)=\bigvee_{u \notin U} C_{u}$ for $U \subseteq \mathcal{P}(\langle n\rangle), U \neq\langle n\rangle$, and $\bar{C}(\langle n\rangle)=*$, and with maps the natural inclusions. For example, when $n=1$ we have

$$
\bar{C}=\left(C_{1} \longleftarrow *\right)
$$

when $n=2$ we have

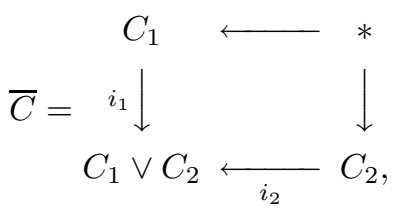

and when $n=3$ we have

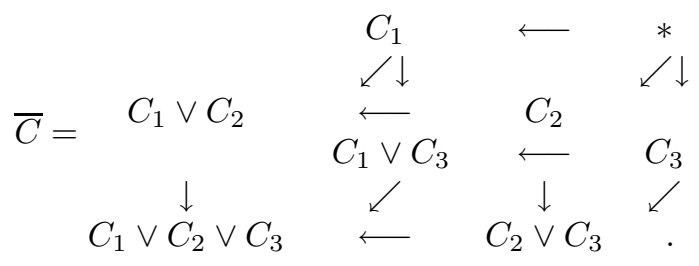

Applying $F$ and taking the total complex, we obtain a chain complex that in degree $k<n$ is

$$
\operatorname{Tot}(F(\bar{C}))_{k}=\bigoplus_{1 \leq i_{1}<i_{2}<\cdots<i_{n-k} \leq n} F\left(C_{i_{1}} \vee C_{i_{2}} \vee \cdots \vee C_{i_{n-k}}\right)
$$

and whose nonzero differentials all have retractions. As a result, one can compute that

$$
\begin{aligned}
H_{k} \operatorname{Tot}(F(\bar{C})) & = \begin{cases}\operatorname{coker}\left(\bigoplus_{i=1}^{n} F\left(C_{1} \vee \ldots \widehat{C_{i}} \cdots \vee C_{n}\right) \rightarrow F\left(\bigvee_{i=1}^{n} C_{i}\right)\right) & \text { if } k=0, \\
0 & \text { if } k \neq 0,\end{cases} \\
& = \begin{cases}\operatorname{cr}_{n} F\left(C_{1}, C_{2}, \ldots, C_{n}\right) & \text { if } k=0, \\
0 & \text { if } k \neq 0 .\end{cases}
\end{aligned}
$$

Thus $\mathrm{cr}_{n} F\left(C_{1}, C_{2}, \ldots, C_{n}\right)$ is naturally quasi-isomorphic to $\operatorname{Tot} F(\bar{C})$.

Considering $c r_{n} F$ as a functor of a single variable, i.e.,

$$
c r_{n} F(C):=c r_{n} F(C, \ldots, C),
$$

one can show that $c r_{p}\left(c r_{q} F\right) \cong c r_{q}\left(c r_{p} F\right)$ for all $p$ and $q$ using this model. For example, if $p=2$ and $q=1$, then $c r_{1} F(C)$ is quasi-isomorphic to the complex

$$
F(C) \leftarrow F(*)
$$

and $c r_{2}\left(c r_{1} F\right)(C)$ is quasi-isomorphic to the total complex of

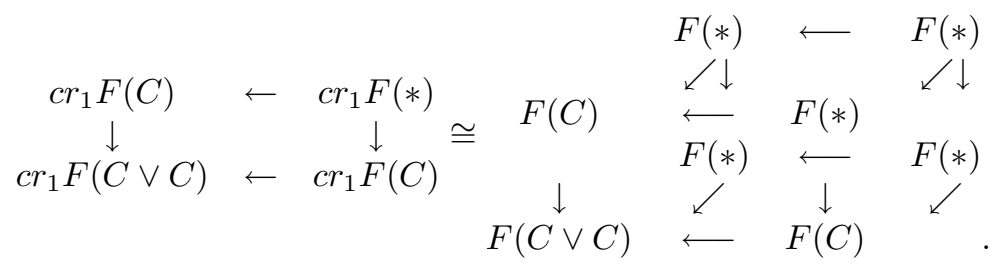


On the other hand,

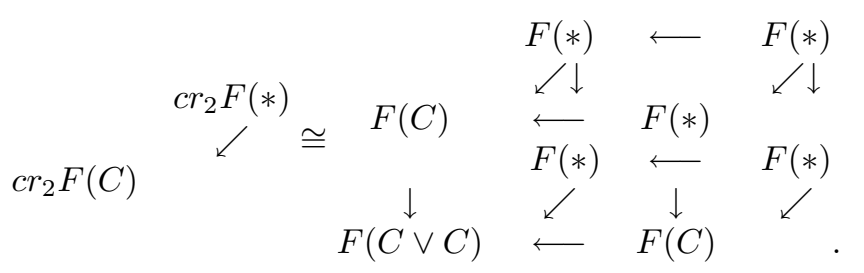

That is, $c r_{2} c r_{1} F(C)$ and $c r_{1} c r_{2} F(C)$ are quasi-isomorphic to the total complex of the same 3-cubical diagram. In general, $c r_{p}\left(c r_{q} F\right)(C)$ and $c r_{q}\left(c r_{p} F\right)(C)$ are both quasi-isomorphic to the complex obtained by applying $F$ to the $(p+q)$-cubical diagram $\bar{C}$ in $\mathcal{C}$ with $\bar{C}(U, V)=\bigvee_{u \notin U}\left(\bigvee_{v \notin V} C\right)$ for $(U, V) \in \mathcal{P}(\langle p\rangle) \times \mathcal{P}(\langle q\rangle)$ and maps given by inclusions of the form $(U \backslash\{i\}) \times V \hookrightarrow U \times V$ and $U \times(V \backslash\{j\}) \hookrightarrow$ $U \times V$.

Note that $c r_{n+1} F \cong 0$ if and only if $c r_{k} F \cong 0$ for all $k \geq n+1$ or, equivalently, if $c r_{n} F$ is a degree 1 functor in each of its $n$ variables separately. If a functor is degree $n$, then we consider it to be degree $k$ for all $k \geq n$ as well. When one composes a degree $n$ and a degree $m$ functor, the result is a functor of degree $m n$, as we prove below.

Proposition 1.6. Let $\mathcal{A}^{\prime}$ be an abelian category. Let $F: \mathcal{A} \rightarrow \mathcal{A}^{\prime}$ be a degree $n$ functor and $G: \mathcal{C} \rightarrow \mathcal{A}$ a degree $m$ functor. The functor $F \circ G: \mathcal{C} \rightarrow \mathcal{A}^{\prime}$ is degree $m n$.

Proof. Let $X_{1}, \ldots, X_{p}$ be objects in $\mathcal{C}$ and $\langle p\rangle=\{1,2, \ldots, p\}$. For $U=\left\{s_{1}, \ldots, s_{t}\right\}$ $\subseteq\langle p\rangle$, let $c r_{U} G$ denote $c r_{t} G\left(X_{s_{1}}, \ldots, X_{s_{t}}\right)$, and for $U=\emptyset$, let $c r_{U} G=G(*)$. By Definition 1.1 and Proposition 1.2 we know that

$$
\begin{aligned}
F \circ G\left(\bigvee_{j=1}^{p} X_{j}\right) & \cong F\left(G(*) \oplus \bigoplus_{\left\{s_{1}, \ldots, s_{t}\right\} \subseteq\langle p\rangle} c r_{t} G\left(X_{s_{1}}, \ldots, X_{s_{t}}\right)\right) \\
& \cong F(*) \oplus \bigoplus_{\left\{U_{1}, \ldots, U_{k}\right\} \subseteq \mathcal{P}(\langle p\rangle)} c r_{k} F\left(c r_{U_{1}} G, \ldots, c r_{U_{k}} G\right) .
\end{aligned}
$$

It follows that

$$
c r_{p}(F \circ G)\left(X_{1}, \ldots, X_{p}\right) \cong \bigoplus_{\left\{U_{1}, \ldots, U_{k}\right\} \subseteq \mathcal{P}(\langle p\rangle), \bigcup_{i=1}^{k} U_{i}=\langle p\rangle} \operatorname{cr}_{k} F\left(c r_{U_{1}} G, \ldots, c r_{U_{k}} G\right) .
$$

Note that the condition that $\bigcup_{i=1}^{k} U_{i}=\langle p\rangle$ comes from the fact that if $\bigcup_{i=1}^{k} U_{i} \neq$ $\langle p\rangle$, then the summand $c r_{k} F\left(c r_{U_{1}} G, \ldots, c r_{U_{k}} G\right)$ is a summand of

$$
F \circ G\left(X_{1} \vee \cdots \vee \widehat{X_{j}} \vee \cdots \vee X_{p}\right)
$$

for some $j$. In this case, the summand is part of a lower order cross effect. Since $G$ is degree $m$ and $F$ is degree $n$, the terms above are nontrivial only when $p \leq m n$. Hence, $F \circ G$ is degree $m n$.

We will consider two adjoint pairs related to the $(n+1)$ st cross effect. The second of these pairs will be used in the next section to define the universal degree $n$ tower. The first pair will be used to construct a related tower. 
Example 1.7 (The adjoint pair $\left(p_{n}, U\right)$ ). Recall that a functor $F: \mathcal{C} \rightarrow \mathcal{A}$ is reduced if $F(*) \cong 0$. Let $F u n c_{*}(\mathcal{C}, \mathcal{A})$ be the category of reduced functors from $\mathcal{C}$ to $\mathcal{A}$ with natural transformations as morphisms, and let $F u n c_{n}(\mathcal{C}, \mathcal{A})$ be the subcategory of reduced degree $n$ functors. Let $U: F u n c_{n}(\mathcal{C}, \mathcal{A}) \rightarrow F u n c_{*}(\mathcal{C}, \mathcal{A})$ be the forgetful functor. The functor $U$ has a left adjoint. To define it, we let + denote the natural transformation from $c r_{n+1} F \rightarrow F$ defined for an object $M$ in $\mathcal{C}$ to be the composite

$$
c r_{n+1} F(M, \ldots, M) \stackrel{i n c}{\longrightarrow} F\left(\bigvee_{i=1}^{n+1} M\right) \stackrel{F(+)}{\longrightarrow} F(M),
$$

where inc denotes the inclusion of $c r_{n+1} F$ as a direct summand of $F\left(\bigvee_{i=1}^{n+1} M\right)$ and + is the fold map. We set

$$
p_{n} F=\operatorname{coker}\left(c r_{n+1} F \stackrel{+}{\rightarrow} F\right)
$$

and leave it to the reader to check that $p_{n}$ is left adjoint to $U$.

We can extend this adjoint pair $\left(p_{n}, U\right)$ to unreduced functors as follows. Every functor $F$ is naturally isomorphic to $F(*) \oplus c r_{1} F$, where $c r_{1} F$ is reduced. Hence, if we define

$$
p_{n} F=\operatorname{coker}\left(c r_{n+1}\left(c r_{1} F\right) \stackrel{+}{\rightarrow} c r_{1} F \stackrel{i n c}{\longrightarrow} F\right),
$$

then we have extended our adjoint pair to all functors.

The adjunction map $F \rightarrow p_{n} F$ (which we will write as $p_{n}$ ) is natural, and is an isomorphism if $F$ is degree $n$. Thus we obtain an inverse limit system:

$$
\begin{aligned}
& \text { F }
\end{aligned}
$$

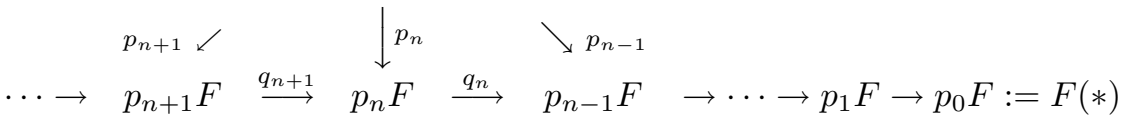

where $p_{n-1}=q_{n}\left(p_{n}\right)$. Clearly, when $F$ is degree $n$, this tower converges to $F$, i.e., the inverse limit of the tower is isomorphic to $F$. In addition, the $p_{n} F$ 's form the 0 th homology of the functors in the tower we describe in section 2 .

Example 1.8 (The adjoint pair $\left(\Delta^{*}, c r_{n+1}\right)$ ). Let $F u n c_{*}\left(\mathcal{C}^{\times n+1}, \mathcal{A}\right)$ be the category of functors of $n+1$ variables from $\mathcal{C}$ to $\mathcal{A}$ that are reduced in each variable separately. Let $\Delta^{*}$ be the functor from $\operatorname{Func}_{*}\left(\mathcal{C}^{\times n+1}, \mathcal{A}\right)$ to $F u n c_{*}(\mathcal{C}, \mathcal{A})$ obtained by composing a functor with the diagonal functor from $\mathcal{C}$ to $\mathcal{C}^{\times n+1}$. The $(n+1)$ st cross effect is the right adjoint to $\Delta^{*}$. The natural isomorphism

$$
\operatorname{Hom}_{F_{u n c}(\mathcal{C}, \mathcal{A})}(F \circ \Delta, G) \cong \operatorname{Hom}_{F u n c_{*}(\mathcal{C} \times n+1, \mathcal{A})}\left(F, c r_{n+1} G\right)
$$

is defined as follows. For objects $M_{1}, \ldots, M_{n+1}$ in $\mathcal{C}$, let $i: F\left(M_{1}, \ldots, M_{n+1}\right) \rightarrow$ $c r_{n+1}(F \circ \Delta)\left(M_{1}, \ldots, M_{n+1}\right)$ be the composite

$$
\begin{gathered}
F\left(M_{1}, \ldots, M_{n+1}\right) \stackrel{F\left(i_{1}, \ldots, i_{n+1}\right)}{\longrightarrow} F\left(\bigvee_{i=1}^{n+1} M_{i}, \ldots, \bigvee_{i=1}^{n+1} M_{i}\right) \\
\stackrel{\pi}{\rightarrow} c r_{n+1}(F \circ \Delta)\left(M_{1}, \ldots, M_{n+1}\right),
\end{gathered}
$$

where $i_{1}, \ldots, i_{n+1}$ are the natural inclusions and $\pi$ projects $F \circ \Delta\left(\bigvee_{i=1}^{n+1} M_{i}\right)$ onto its summand $c r_{n+1}(F \circ \Delta)\left(M_{1}, \ldots, M_{n+1}\right)$. Then, the isomorphism (1.9) takes a natural transformation $\beta: F \circ \Delta \rightarrow G$ to the composite

$$
F \stackrel{i}{\rightarrow} c r_{n+1}(F \circ \Delta) \stackrel{c r_{n+1} \beta}{\rightarrow} c r_{n+1} G .
$$


And, for an object $M$ in $\mathcal{C}$, a natural transformation $\alpha: F \rightarrow c r_{n+1} G$ is sent to the natural composite

$$
F(M, \ldots, M) \stackrel{\alpha}{\rightarrow} c r_{n+1} G(M, \ldots, M) \stackrel{i n c}{\rightarrow} G\left(\bigvee_{i=1}^{n+1} M\right) \stackrel{G(+)}{\rightarrow} G(M),
$$

where $i n c$ and + are the transformations used in the previous example. Note that

$$
p_{n}=\text { cokernel }\left(\Delta^{*} \circ c r_{n+1} \stackrel{\text { coadj }}{\longrightarrow} \mathrm{id}\right),
$$

where coadj denotes the counit of the adjunction.

\section{Cotriple COnstructions And DEGReE $n$ FunCtors}

In Example 1.7 we discussed how to create a degree $n$ functor $p_{n} F$ from an arbitrary functor $F$. As we indicated in the introduction, $p_{n}$ is a right exact functor of functors, but is not exact. In this section, we construct, for each $n>0$ and functor $F$, a functor $P_{n} F$ that is degree $n$ up to quasi-isomorphism, and show that $P_{n}$ is an exact functor of functors. Moreover, we build an inverse limit system, similar to that of Example 1.7, comprising the functors $P_{1} F, P_{2} F, \ldots, P_{n-1} F, P_{n} F, \ldots$ and natural transformations from $F$ to each of these functors. We prove that the $P_{n} F$ 's are universal among degree $n$ functors with natural transformations from $F$. Our constructions rely on the use of a cotriple arising from the adjoint pair of Example 1.8. We begin this section by reviewing some facts about cotriples needed for the definitions of $P_{n} F$ and the universal degree $n$ tower. Further details about cotriples can be found in [We, §8.6]. Throughout this section, unless otherwise noted, $\mathcal{C}$ is a basepointed category with finite coproducts, $\mathcal{A}$ is an abelian category, and $\mathcal{B}$ is an arbitrary category.

Definition 2.1. A cotriple (or comonad) $(\perp, \epsilon, \delta)$ in the category $\mathcal{B}$ is a functor $\perp: \mathcal{B} \rightarrow \mathcal{B}$ together with natural transformations $\epsilon: \perp \Rightarrow \operatorname{id}_{\mathcal{B}}$ and $\delta: \perp \Rightarrow \perp \perp$ such that the following diagrams commute:

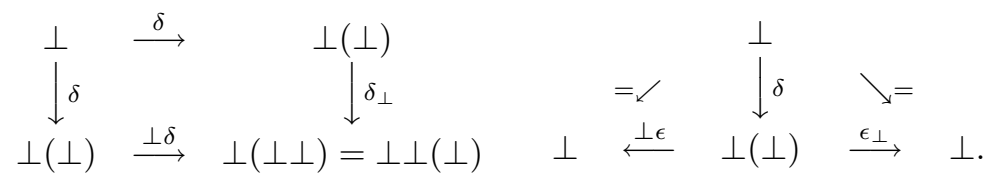

Cotriples often arise from adjoint pairs.

Example 2.2. Let $(F, U)$ be a pair of adjoint functors and $\perp=F U$. Let $\epsilon$ be a counit and $\eta$ a unit for the adjoint pair. Let $\eta_{U}$ be the natural transformation that for an object $B$ is given by $\eta_{U(B)}: U(B) \rightarrow U F(U(B))$. Then $\left(\perp, \epsilon, F\left(\eta_{U}\right)\right)$ is a cotriple. In particular, the adjoint pair of Example 1.8 yields the cotriple $\Delta^{*} \circ \mathrm{cr}_{n+1}$.

Cotriples yield simplicial objects in the following manner.

Remark 2.3. Let $(\perp, \epsilon, \delta)$ be a cotriple in $\mathcal{B}$ and let $B$ be an object in $\mathcal{B}$. Then $\perp^{*+1} B$ is the following simplicial object in $\mathcal{B}$ :

$$
\begin{gathered}
{[n] \mapsto \perp^{(n+1)} B=\overbrace{\perp \cdots \perp}^{n+1 \text { times }} B,} \\
d_{i}=\perp^{(i)} \epsilon \perp^{(n-i)}: \quad \perp^{(n+1)} B \rightarrow \perp^{(n)} B, \\
s_{i}=\perp^{(i)} \delta \perp^{(n-i)}: \quad \perp^{(n+1)} B \rightarrow \perp^{(n+2)} B .
\end{gathered}
$$


Observe that $\perp^{*+1}$ is augmented over $\operatorname{id}_{\mathcal{B}}$ by $\epsilon$. In particular, if we consider $\left(\mathrm{id}_{\mathcal{B}}, \mathrm{id}, \mathrm{id}\right)$ as the trivial cotriple, then $\epsilon$ gives a natural simplicial map from $\perp^{*+1}$ to $\mathrm{id}^{*+1}$, where $\mathrm{id}^{*+1}$ is the trivial simplicial $\mathcal{B}$-object.

Using $\perp^{*+1}$ and the augmentation, one can construct the following chain complex for the abelian category $\mathcal{A}$.

Definition 2.4. Let $(\perp, \epsilon, \delta)$ be a cotriple on an abelian category $\mathcal{A}$ and let $A$ be an object in $\mathcal{A}$. Then $C_{*}^{\perp}(A)$ is the chain complex with

$$
C_{n}^{\perp}(A)= \begin{cases}A & \text { if } n=0, \\ \perp^{n} A & \text { if } n>0,\end{cases}
$$

and $\partial_{n}: C_{n}^{\perp}(A) \rightarrow C_{n-1}^{\perp}(A)$ defined by

$$
\partial_{n}= \begin{cases}\epsilon & \text { if } n=1 \\ \sum_{i=0}^{n-1}(-1)^{i} d_{i} & \text { if } n>1\end{cases}
$$

The chain complex $C_{*}^{\perp}(A)$ is the mapping cone of the composition

$$
C\left(\perp^{*+1} A\right) \stackrel{\epsilon}{\longrightarrow} C\left(\mathrm{id}^{*+1} A\right) \stackrel{\simeq}{\longrightarrow} N\left(\mathrm{id}^{*+1} A\right)=A
$$

where $C\left(\perp^{*+1} A\right)$ and $C\left(\mathrm{id}^{*+1} A\right)$ are the chain complexes associated to $\perp^{*+1} A$ and $\mathrm{id}^{*+1} A$, respectively, and $N\left(\mathrm{id}^{*+1} A\right)$ is the normalized chain complex associated to $\mathrm{id}^{*+1} A$. (See [We, $\left.§ 8.2-3\right]$ for definitions of $C$ and $N$.)

For an object $A$ in $\mathcal{A}$, the chain complex $C_{*}^{\perp}(A)$ is acyclic whenever $\epsilon: \perp A \rightarrow A$ has a section. This is a consequence of the next proposition and will be useful in proving several results concerning the functor $P_{n} F$. (For readers familiar with cotriples, these results are related to the fact that $\perp^{*+1} A \stackrel{\epsilon}{\longrightarrow} A$ is aspherical when $A$ is a $\perp$-projective object in $\mathcal{A}$. See [We, 8.6.8].)

Proposition 2.5. Let $(\perp, \epsilon, \delta)$ be a cotriple on an abelian category $\mathcal{A}$, and let $A$ be an object in $\mathcal{A}$. For any $h \in \operatorname{Hom}_{\mathcal{A}}(A, \perp A), C_{*}^{\perp}(\epsilon \circ h)$ is chain homotopic to the zero map on $C_{*}^{\perp}(A)$.

Proof. We begin by noting that the diagram

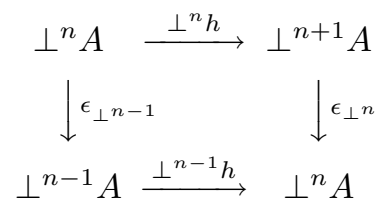

commutes by naturality of $\epsilon$ for all $n \geq 1$. Thus, $\epsilon_{\perp^{n}} \circ \perp^{n} h=\perp^{n-1} h \circ \epsilon_{\perp^{n-1}}$ for all $n \geq 1$. One can use this to show that the maps $k_{n}: \perp^{n} A \rightarrow \perp^{n+1} A$ defined by $k_{n}=(-1)^{n} \perp^{n} h$ form a chain homotopy between $C_{*}^{\perp}(\epsilon \circ h)$ and the zero map.

Corollary 2.6. 1) If $\epsilon: \perp A \rightarrow A$ has a section, then $C_{*}^{\perp}(A) \simeq 0$.

2) If $A=\perp B$ for some object $B$ in $\mathcal{A}$, then $C_{*}^{\perp}(A) \simeq 0$.

Proof. 1) is a direct consequence of the proposition. To see that 2) is true, note that $\delta$ is a section to $\epsilon_{\perp}$ by the definition of cotriple.

We are also able to show that $\perp C_{*}^{\perp}(A)$ is an acyclic chain complex. 
Proposition 2.7. Let $(\perp, \epsilon, \delta)$ be a cotriple in the abelian category $\mathcal{A}$. For any object $A$ in $\mathcal{A}$, the chain complex $\perp\left(C_{*}^{\perp}(A)\right)$, obtained by applying $\perp$ to $C_{*}^{\perp}(A)$ degreewise, is acyclic.

Proof. The simplicial object $\perp\left(\perp_{n+1}^{*+1} A\right)$ is the path space of $\perp_{n+1}^{*+1} A$, and the path space is homotopy equivalent to the constant simplicial object $\perp A$ (see We pp. 269-270]). This homotopy equivalence yields the desired chain homotopy between the identity and the zero map on $\perp\left(C_{*}^{\perp}(A)\right)$. In particular, one can show that the maps

$$
\delta_{\perp^{n}}: \perp^{n+1} A \rightarrow \perp^{n+2} A
$$

form a chain contraction of the identity on $\perp\left(C_{*}^{\perp}(A)\right)$, using the fact that, for any $t \in \operatorname{Hom}\left(\perp^{n+2-k} A, \perp^{n+1-k} A\right)$,

$$
\delta_{\perp^{n}} \circ \perp^{k} t=\perp^{k+1} t \circ \delta_{\perp^{n+1}} .
$$

With this, we are able to define $P_{n} F$ and prove that it is a degree $n$ functor up to quasi-isomorphism. In doing so, we will be working with a cotriple in the abelian category $\operatorname{Func}_{*}(\mathcal{C}, \mathcal{A})$ of reduced functors from $\mathcal{C}$ to $\mathcal{A}$.

Definition 2.8. Let $\perp_{n+1}=\Delta^{*} \circ \mathrm{cr}_{n+1}$ be the cotriple on $F u n c_{*}(\mathcal{C}, \mathcal{A})$ obtained from the adjoint pair $\left(\Delta^{*}, c r_{n+1}\right)$ of Example 1.8. We define $P_{n}$ to be the functor from $\operatorname{Func}_{*}(\mathcal{C}, \mathcal{A})$ to $\operatorname{Func}_{*}\left(\mathcal{C}, C h_{\geq 0} \mathcal{A}\right)$ given by

$$
P_{n}=\text { Mapping Cone }\left[N\left(\perp_{n+1}^{*+1}\right) \stackrel{\epsilon}{\longrightarrow} N\left(\mathrm{id}^{*+1}\right)=\mathrm{id}\right],
$$

where $N$ is the associated normalized chain complex of the simplicial object. We let $p_{n}$ : id $\rightarrow P_{n}$ be the natural transformation obtained from the mapping cone. Note that this $p_{n}$ is different from the $p_{n}$ defined in section 1 . Our desire to be consistent with notation elsewhere has led to this abuse of notation, but we hope that the meaning of $p_{n}$ will be clear from context. For a functor $F$ in $F u n c_{*}(\mathcal{C}, \mathcal{A}), P_{n} F$ is naturally chain homotopy equivalent to the chain complex $C_{*}^{\perp_{n+1}}(F)$. Both of these models will be important for subsequent results. We will use them interchangeably.

We extend the definition of $P_{n} F$ from the category of reduced functors to the category $\operatorname{Func}(\mathcal{C}, \mathcal{A})$ of all functors from $\mathcal{C}$ to $\mathcal{A}$ as follows. Every functor $F$ is naturally isomorphic to $F(*) \oplus c r_{1} F$, where $c r_{1} F$ is reduced. We define

$$
P_{n} F=\text { Mapping Cone }\left[N\left(\perp_{n+1}^{*+1} c r_{1} F\right) \stackrel{\epsilon}{\longrightarrow} N\left(\mathrm{id}^{*+1} c r_{1} F\right)=c r_{1} F \stackrel{i n c}{\longrightarrow} F\right] .
$$

We can also extend the definition of $P_{n}$ to functors from $\mathcal{C}$ to $C h_{>0} \mathcal{A}$. To do so, note that any functor $F_{*}: \mathcal{C} \rightarrow C h_{>0} \mathcal{A}$ is equivalent to a chain complex of functors from $\mathcal{C}$ to $\mathcal{A}$. Applying $P_{n}$ to each functor in the chain complex, we obtain a bicomplex of functors. Taking the total complex (using direct sums and the sign convention of Remark 2.5 in [J-M1] produces a new functor from $\mathcal{C}$ to $C h_{>0} \mathcal{A}$ that we will call $P_{n} F_{*}$. When $\mathcal{A}$ is cocomplete, $P_{n} F$ can also be defined in this manner for functors $F: \mathcal{C} \rightarrow C h \mathcal{A}$, where $C h \mathcal{A}$ denotes the category of unbounded chain complexes over $\mathcal{A}$.

We now focus our attention on those properties of $P_{n} F$ of greatest interest to us - that the $P_{n} F$ 's form a tower of universal degree $n$ functors similar to Goodwillie's Taylor tower of $n$-excisive functors. The functor $P_{n} F$ is degree $n$ in the following sense. 
Definition 2.9. Given a functor $F$ from $\mathcal{C}$ to $C h \mathcal{A}$, we say that $F$ is degree $n$ if $c r_{n+1} F$ is acyclic as a functor from $\mathcal{C}^{\times n+1}$ to $C h \mathcal{A}$. That is, $c r_{n+1} F$ has no homology when evaluated on a collection of $n+1$ objects in $\mathcal{C}$. We say that $F$ is strictly degree $n$ if $c r_{n+1} F \cong 0$.

Note. Technically this terminology contradicts our definition of degree $n$ in section 1 , as $C h(\mathcal{A})$ is an additive category. However, when we write $C h(\mathcal{A})$ we are implicitly considering this as a category with a localizing subcategory of weak equivalences determined by quasi-isomorphism. We choose our terminology so that degree $n$ reflects this additional structure, and trust that no serious confusion will arise in practice.

Proposition 2.10. Let $\mathcal{A}^{\prime}$ be an abelian category, $F: C h \mathcal{A} \rightarrow C h \mathcal{A}^{\prime}$ and $G: \mathcal{C} \rightarrow$ $C h \mathcal{A}$. If $F$ is degree $n$ and $G$ is degree $m$, then $F \circ G$ is degree $m n$.

Proof. The proof is similar to that of Proposition 1.6.

Lemma 2.11. Let $F: \mathcal{C} \rightarrow C h_{\geq 0} \mathcal{A}$ (or $F: \mathcal{C} \longrightarrow C h \mathcal{A}$ for $\mathcal{A}$ cocomplete).

1) The functor $P_{n} F$ is degree $n$.

2) If $F$ is degree $n$, then $p_{n}: F \rightarrow P_{n} F$ is a quasi-isomorphism.

3) The pair $\left(P_{n}, p_{n}\right)$ is universal up to natural quasi-isomorphism with respect to degree $n$ functors with natural transformations from $F$.

Proof. For each $k, \perp_{n+1}\left(P_{n} F_{k}\right)$ is acyclic by Proposition 2.7. It follows that $c r_{n+1} P_{n} F_{k}\left(X_{1}, \ldots, X_{n+1}\right)$ is acyclic for any $X_{1}, \ldots, X_{n+1}$ by setting $X=\bigvee_{i=1}^{n+1} X_{i}$ and noting that $c r_{n+1} P_{n} F_{k}\left(X_{1}, \ldots, X_{n+1}\right)$ is a direct summand of $\perp_{n+1}\left(P_{n} F_{k}\right)(X)$. Then $c r_{n+1}\left(P_{n} F\right)$ is the total complex of a bicomplex supported in quadrants I and IV with acyclic rows, and so is itself acyclic.

If $F$ is degree $n$, then $\perp_{n+1} F$ is acyclic. Since $\perp_{n+1}$ is exact, it follows that $\perp_{n+1}^{k} F$ is acyclic for all $k \geq 1$. As a consequence, $C\left(\perp_{n+1}^{*+1} F\right)$ is an acyclic chain complex, and since $N\left(\perp_{n+1}^{*+1} F\right) \simeq C\left(\perp_{n+1}^{*+1} F\right)$ ([W], Theorem 8.3.8), $N\left(\perp_{n+1}^{*+1} F\right)$ is acyclic as well. Recall that

$$
P_{n} F=\text { Mapping Cone }\left[N\left(\perp_{n+1}^{*+1} F\right) \longrightarrow F\right]
$$

and $p_{n}: F \longrightarrow P_{n} F$ is the natural transformation associated to the mapping cone. Since $N\left(\perp_{n+1}^{*+1} F\right) \simeq 0$, it follows from properties of mapping cones that $p_{n}: F \longrightarrow P_{n} F$ is a quasi-isomorphism.

Part 3) follows by an argument similar to that used to prove Proposition 1.18 of G1]. Let $\tau: F \rightarrow G$ be a natural transformation from $F$ to a degree $n$ functor $G$. Considering the commutative diagram

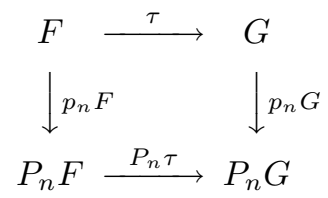

one sees that, up to quasi-isomorphism, $\tau$ factors through $p_{n} F$, since $p_{n} G$ is a quasi-isomorphism. To see that this factorization is unique, consider the diagram

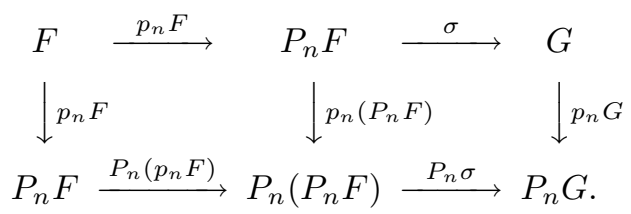


If $\sigma \circ p_{n} F=\tau$, then $P_{n} \sigma \circ P_{n}\left(p_{n} F\right)=P_{n}\left(\sigma \circ p_{n} F\right)$ must equal $P_{n}(\tau)$. It is straightforward to show that $p_{n}\left(P_{n} F\right) \simeq P_{n}\left(p_{n} F\right)$. Then $p_{n} G, P_{n}\left(p_{n} F\right)$, and $p_{n}\left(P_{n} F\right)$ are all quasi-isomorphisms, and it follows that $\sigma$ is uniquely determined by $P_{n} \tau$ (and hence $\tau$ ) up to quasi-isomorphism.

By Lemma 2.11 we obtain, up to quasi-isomorphism, natural transformations $q_{n}: P_{n} \rightarrow P_{n-1}$ that produce the desired tower of functors. For a functor $F$, $q_{n} F$ is obtained by composing $P_{n}\left(p_{n-1} F\right)$ with the inverse of the natural quasiisomorphism $P_{n-1} F \stackrel{p_{n} P_{n-1} F}{\longrightarrow} P_{n} P_{n-1} F$. However, $q_{n}$ can also be obtained as an explicit natural transformation. To do so, first note that if we have a natural transformation $\eta: \perp_{n+1} \rightarrow \perp_{n}$ of cotriples, we can define a natural transformation from $P_{n}$ to $P_{n-1}$ by using the chain map given by

$$
\eta([k])=\perp_{n}^{k-1}\left(\eta_{\perp_{n+1}}\right) \circ \perp_{n}^{k-2}\left(\eta_{\perp_{n+1}^{2}}\right) \circ \cdots \circ \eta_{\perp_{n+1}^{k}} .
$$

Then $q_{n}: P_{n} \rightarrow P_{n-1}$ is the natural transformation determined by the natural composite:

$$
\begin{aligned}
& q_{n}: c r_{n+1} F(M, M \ldots, M) \stackrel{i n c}{\longrightarrow} c r_{n} F(M \vee M, M, \ldots, M) \\
& c r_{n} F\left(\stackrel{+, i d, \ldots, i d)}{\longrightarrow} c r_{n} F(M, \ldots, M) .\right.
\end{aligned}
$$

Hence we have the following theorem.

Theorem 2.12. Given a functor $F$ from $\mathcal{C}$ to $C h_{\geq 0} \mathcal{A}$, there is a natural tower of functors:

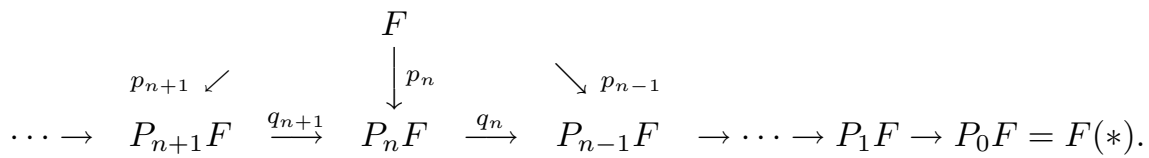

The 0th homology of this tower is the tower of Example 1.7 for $H_{0}(F)$. The pairs $\left(P_{n}, p_{n}\right)$ are universal (up to natural quasi-isomorphism) with respect to maps from $F$ to degree $n$ functors. The result holds for $F: \mathcal{C} \rightarrow C h \mathcal{A}$ when $\mathcal{A}$ is cocomplete.

As indicated above, for a functor $F$ from $\mathcal{C}$ to $\mathcal{A}, H_{0} P_{n} F$ is the functor $p_{n} F$ defined in Example 1.7. Note that $p_{n}$ does not preserve short exact sequences of functors. In particular, for a short exact sequence (that is, short exact upon evaluation at any given object)

$$
0 \rightarrow F \rightarrow G \rightarrow H \rightarrow 0
$$

of functors from $\mathcal{C}$ to $\mathcal{A}$, the snake lemma yields an exact sequence

$$
\begin{aligned}
\operatorname{ker}\left(\perp_{n+1} F \rightarrow F\right) & \rightarrow \operatorname{ker}\left(\perp_{n+1} G \rightarrow G\right) \rightarrow \operatorname{ker}\left(\perp_{n+1} H \rightarrow H\right) \\
& \rightarrow p_{n} F \rightarrow p_{n} G \rightarrow p_{n} H \rightarrow 0 .
\end{aligned}
$$

There are many examples for which $\operatorname{ker}\left(\perp_{n+1} H \rightarrow H\right)$ is not zero (e.g., $H=T^{n+1}$ ) and so the sequence $p_{n} F \rightarrow p_{n} G \rightarrow p_{n} H$ is not exact. However, $P_{n}$ preserves short exact sequences.

Proposition 2.13. 1) For any functor $F: \mathcal{C} \rightarrow \mathcal{A}$ we have $H_{0}\left(P_{n} F\right)=p_{n} F$, the functor defined in Example 1.\%.

2) If $F \rightarrow G \rightarrow H$ is a short exact sequence of functors, then $P_{n} F \rightarrow P_{n} G \rightarrow$ $P_{n} H$ is a short exact sequence of chain complexes of functors.

3) The functor $P_{n}$ preserves quasi-isomorphisms of functors. 
Proof. The proof of 1) follows by definition. The proof of 2) follows from the definition of $P_{n}$ and the fact that $\perp_{n+1}$ is exact. For a natural transformation $F \stackrel{\eta}{\longrightarrow} G$ that is a quasi-isomorphism on all objects in $\mathcal{C}, 3$ ) follows from 2) by using the short exact sequence associated with the mapping cone of $\eta$ and showing that $P_{n}$ preserves acyclicity.

We conclude this section with some examples and comparisons with related functors.

Examples and Remarks 2.14. 1) The functor $P_{1} F$ is a reformulation and extension of Mac Lane's $Q$-construction ([E-M1]). That is, when $F=S[-]: \mathcal{M}_{R} \rightarrow \mathcal{M}_{S}$ is the functor that takes an $R$-module to the free $S$-module generated by its underlying set, $P_{1} S[-]$ is isomorphic to Mac Lane's $Q$-construction for the rings $R$ and $S$. For an $R$-module $M$, the $Q$-construction in this case is an explicit chain complex whose homology is the stable homology of $M$ with coefficients in $S$, i.e., the homotopy of the smash product of the Eilenberg-Mac Lane spectra HM and HS. More generally, for an arbitrary functor $F$ from $\mathcal{M}_{R}$ to $\mathcal{M}_{S}, P_{1} F$ is isomorphic to the generalization of Mac Lane's $Q$-construction found in [J-P]. The isomorphism comes from a direct comparison of the two chain complexes. For example, the degeneracies of $\perp_{n+1}^{*+1}$ correspond to the slabs of the $Q$-construction.

2 ) When $F$ is the simplicial prolongation of a functor, the homology groups of $P_{1} F$ are isomorphic to the stable derived functors of $F$ as defined by Dold and Puppe ([D-P]). That is, $P_{1} F$ is equivalent to a direct limit of functors of the form $\Omega^{n} F B^{n}$, where $\Omega$ is the loop space functor and $B$ is the bar construction. See J-M1 for more details.

3) For an arbitrary $n, P_{n} F$ is isomorphic to the $P_{n} F$ defined in J-M2 for the same reasons that $P_{1} F$ was isomorphic to the $Q$-construction. In [J-M2], the functors $P_{n} F$ were used to obtain explicit models for Goodwillie's $n$th degree Taylor polynomial approximations to functors in certain cases. In particular, given a functor $F$ from $\mathcal{M}_{R}$ to $\mathcal{M}_{S}$, we can construct a corresponding functor of spaces $\widehat{F}$. For such functors we show that $P_{n} \widehat{F}$, the degree $n$ term in Goodwillie's Taylor tower for $\widehat{F}$ (as defined in G3]), is naturally equivalent to $\widehat{P_{n} F}$, the functor of spaces arising from the functor $P_{n} F$ defined above. In general, the Taylor tower defined in this section is not the same as Goodwillie's tower. Our tower is a tower of degree $n$ functors, whereas Goodwillie's tower consists of $n$-excisive functors. See section 5 of [J-M2 for details.

4) By Example 1.4.1 and Lemma 2.11, $P_{n} F \cong F$ when $F$ is the $n$-fold tensor product, symmetric power, or exterior power functor. Simson and Tyc ([S], $[\underline{S-T}])$, Betley $([\mathrm{Be}])$, and Bousfield ( $[\mathrm{Bo}])$ have determined the homology of $P_{1} F$ in some cases when $F$ is the $n$th symmetric power or exterior power functor. Section 4 of J-M1] contains a summary of Simson and Tyc's calculations.

5) The Taylor tower for the functor Exp of Example 1.4.3 is studied in section 6.

6) In the next section (Proposition 3.2) we will prove that for any functor $F: \mathcal{C} \rightarrow$ $C h \mathcal{A}$ ( $\mathcal{A}$ is cocomplete), $P_{k} \perp_{n} F \simeq 0$ if $k<n$. From this, it follows that for $k<n$, $P_{k} P_{n} F$ is the total complex of a bicomplex whose bottom row is $P_{k} F$ and whose other rows are acyclic. Hence $P_{k} F \simeq P_{k} P_{n} F$ for $k<n$. This quasi-isomorphism is given by the natural transformation $P_{k}\left(p_{n} F\right)$. 
7) The $n$th layer of the tower of Theorem 2.12 is the functor $D_{n} F: \mathcal{C} \rightarrow C h \mathcal{A}$ given by

$$
D_{n} F=\operatorname{fiber}\left(P_{n} F \stackrel{q_{n}}{\longrightarrow} P_{n-1} F\right),
$$

where the fiber is the homotopy fiber, i.e., the mapping cone shifted down one degree. Note that $D_{n} F, P_{n} F$, and $P_{n-1} F$ form a long exact sequence in homology

$$
\cdots \rightarrow H_{*} D_{n} F \rightarrow H_{*} P_{n} F \rightarrow H_{*} P_{n-1} F \rightarrow H_{*-1} D_{n} F \rightarrow \cdots .
$$

It is straightforward to show that $D_{n} F$ is a degree $n$ functor. Moreover, $P_{k} D_{n} F \simeq 0$ for $k<n$. To see this, note that by the previous example (and Lemma 2.11 in the case where $k=n-1$ ), we have

$$
\begin{aligned}
P_{k} D_{n} F & =P_{k} \operatorname{fiber}\left(P_{n} F \rightarrow P_{n-1} F\right) \\
& \cong \operatorname{fiber}\left(P_{k} P_{n} F \rightarrow P_{k} P_{n-1} F\right) \\
& \simeq \operatorname{fiber}\left(P_{k} F \rightarrow P_{k} F\right) \\
& \simeq 0 .
\end{aligned}
$$

\section{HomogeneOUs DEGREE $n$ FUnCTORS}

Our next goal is to determine the general form of the layers of our tower, i.e., the functors $D_{n} F$ defined in Example 2.14.7. We prove that for each $n, D_{n} F$ is naturally equivalent to the homotopy orbits (with respect to a symmetric group action) of the functor obtained by applying $D_{1}$ to each variable of $c r_{n} F$. This characterization of the layers was inspired by a similar characterization established by Goodwillie for his Taylor tower for functors of spaces ([G3]). To prove this result in our setting, we first prove some general facts about the diagonals of functors of $n$ variables. We then review the definition and some basic properties of homotopy orbits before proving the desired result for $D_{n} F$. We conclude the section with a remark indicating why homotopy orbits arise in the layers. Throughout this section, $\mathcal{C}$ is a basepointed category with finite coproducts and $\mathcal{A}$ is a cocomplete abelian category. The results in this section are stated for functors to $C h \mathcal{A}$, but also hold for functors to $C h_{\geq 0} \mathcal{A}$ when $\mathcal{A}$ is not cocomplete.

The $n$th cross effect functor is a functor of $n$ variables. We single out for special attention the following properties that functors of $n$ variables may have, and new functors that can be built from functors of $n$ variables.

Definition 3.1. Let $G$ be a functor from $\mathcal{C}^{\times n}$ to $C h \mathcal{A}$.

1) The diagonal of $G$ is the functor $G^{\Delta}=G \circ \Delta: \mathcal{C} \rightarrow C h \mathcal{A}$ obtained by composition with the diagonal functor.

2) If $G\left(M_{1}, \ldots, M_{n}\right) \simeq 0$ whenever any $M_{i}=*$, we say that $G$ is $n$-multireduced.

3) $G$ is $n$-multiadditive if $G$ is $n$-multireduced and degree 1 in each of its variables.

4) The $n$-multilinearization of $G, D_{1}^{(n)} G$, is the functor that results when $D_{1}$ is applied to each variable of $G$ separately. That is, $D_{1}^{(n)} G=D_{1}^{1} D_{1}^{2} D_{1}^{3} \ldots D_{1}^{n} G$, where $D_{1}^{k} G$ is the functor obtained by holding all but the $k$ th variable of $G$ constant and applying $D_{1}$ to this functor of one variable.

We will ultimately show that $D_{n} F$ is equivalent to the homotopy orbits of $D_{1}^{(n)} c r_{n} F$. The first step in this process is to analyze $P_{k} G^{\Delta}$ for an $n$-multireduced functor $G$. 
Proposition 3.2. For an n-multireduced functor $G: \mathcal{C}^{\times n} \rightarrow C h \mathcal{A}$,

$$
P_{k} G^{\Delta} \simeq \begin{cases}0 & \text { if } k<n, \\ \left(D_{1}^{(n)} G\right)^{\Delta} & \text { if } k=n .\end{cases}
$$

We prove that $P_{k} G^{\Delta} \simeq 0$ for $k<n$ in the next lemma. This and the following lemma, which implies that $D_{1}^{(n)} G^{\Delta}$ is degree $n$, are used to prove the remainder of Proposition 3.2.

Lemma 3.3. Let $G: \mathcal{C}^{\times n} \rightarrow C h \mathcal{A}$ be an $n$-multireduced functor. For $k<n$, $P_{k} G^{\Delta} \simeq 0$.

Proof. Fix $t<n+1$. We prove this result by constructing a section to $\perp_{t} G^{\Delta} \rightarrow G^{\Delta}$ and applying Corollary 2.6. For any object $M$ in $\mathcal{C}$, let $\eta_{t}: G^{\Delta}(M) \longrightarrow G^{\Delta}\left(\bigvee_{t} M\right)$ be the map defined by

$$
\eta_{t}=G\left(e_{1}, e_{2}, \ldots, e_{t-1}, e_{t}, e_{t}, \ldots, e_{t}\right),
$$

where $e_{i}: M \rightarrow \bigvee_{t} M$ is the standard inclusion into the $i$ th position. Observe that for the fold map $+: \bigvee_{t} M \rightarrow M$,

$$
G^{\Delta}(+) \circ \eta_{t}=\operatorname{id}_{G^{\Delta}} .
$$

We claim that $\eta_{t}$ yields a natural section $\widehat{\eta}_{t}: G^{\Delta} \rightarrow c r_{t} G^{\Delta}$ to the map $+: c r_{t} G^{\Delta} \rightarrow$ $G^{\Delta}$ defined in Example 1.7. To see this, consider the decomposition of $G^{\Delta}\left(\bigvee_{t} M\right)$ as a sum of cross effects:

$$
G^{\Delta}\left(\bigvee_{t} M\right) \cong \bigoplus_{\left\{s_{1}, s_{2}, \ldots, s_{p}\right\} \subseteq\{1,2, \ldots, t\}} c r_{p} G^{\Delta}(M)
$$

We are interested in the image of $\eta_{t}$ in each of these summands. In particular, we claim that for $p<t$ the image of $\eta_{t}$ in each $c r_{p} G^{\Delta}(M)$ is 0 . This follows by noting that for $p<t$, the composite

$$
G^{\Delta}(M) \stackrel{\eta_{t}}{\longrightarrow} G^{\Delta}\left(\bigvee_{t} M\right) \longrightarrow G^{\Delta}\left(\bigvee_{p} M\right)
$$

given by any of the natural projections $\bigvee_{t} M \longrightarrow \bigvee_{p} M$ is the zero map, since $G$ is multireduced. Since $c r_{p} G^{\Delta}(M)$ is a direct summand of $G^{\Delta}\left(\bigvee_{p} M\right)$, the image of $\eta_{t}$ in $c r_{p} G^{\Delta}(M)$ is 0 for each copy of $c r_{p} G^{\Delta}(M)$ in $G^{\Delta}\left(\bigvee_{t} M\right)$. Thus the image of $\eta_{t}$ is contained entirely in $c r_{t} G^{\Delta}(M)$, and so $\widehat{\eta}_{t}: G^{\Delta} \rightarrow c r_{t} G^{\Delta}$ is a section to $+: c r_{t} G^{\Delta} \rightarrow G^{\Delta}$. Hence $P_{t-1} G^{\Delta} \simeq 0$ by Corollary 2.6 .

Lemma 3.4. If $H: \mathcal{C}^{\times n} \longrightarrow C h \mathcal{A}$ is an n-multiadditive functor, then $H^{\Delta}$ is degree $n$.

Proof. Let $M_{1}, \ldots, M_{t}$ be objects in $\mathcal{C}$. Since $H$ is multiadditive, we have a natural quasi-isomorphism

$$
H^{\Delta}\left(M_{1} \vee \cdots \vee M_{t}\right) \simeq \bigoplus_{\alpha \in \operatorname{Hom}(\langle n\rangle,\langle t\rangle)} H\left(M_{\alpha(1)}, \ldots, M_{\alpha(n)}\right),
$$

where $\operatorname{Hom}(\langle n\rangle,\langle t\rangle)$ denotes the set of maps from $\langle n\rangle=\{1,2, \ldots, n\}$ to $\langle t\rangle=$ $\{1,2, \ldots, t\}$. If $\alpha \in \operatorname{Hom}(\langle n\rangle,\langle t\rangle)$ is not a surjection, then $H\left(M_{\alpha(1)}, \ldots, M_{\alpha(n)}\right)$ is 
a direct summand of $H^{\Delta}\left(M_{1} \vee M_{2} \vee \ldots \widehat{M}_{i} \cdots \vee M_{t}\right)$ for some $i$, and so is part of a cross effect of order less than $t$. Hence,

$$
c r_{t} H^{\Delta}\left(M_{1}, \ldots, M_{t}\right) \simeq \bigoplus_{\beta \in \operatorname{Surj}(\langle n\rangle,\langle t\rangle)} H\left(M_{\beta(1)}, \ldots, M_{\beta(n)}\right) .
$$

Thus, when $t>n, c r_{t} H^{\Delta} \simeq 0$ and $H$ is degree $n$.

We now examine $P_{n} G^{\Delta}$ and prove Proposition 3.2.

Proof (of Proposition 3.2). Let $p(n): G^{\Delta} \rightarrow\left(D_{1}^{(n)} G\right)^{\Delta}$ be the natural transformation that is $p_{1}$ in each variable of $D_{1}^{(n)} G$. In other words, $p(n)$ is the composite

$$
G \stackrel{p_{1}}{\longrightarrow} D_{1}^{n} G \stackrel{p_{1}}{\longrightarrow} D_{1}^{n-1} D_{1}^{n} G \stackrel{p_{1}}{\longrightarrow} \ldots \stackrel{p_{1}}{\longrightarrow} D_{1}^{1} \ldots D_{1}^{n} G=D_{1}^{(n)} G .
$$

Consider the natural commuting diagram

$$
\begin{array}{cc}
G^{\Delta} \stackrel{p(n)}{\longrightarrow} & \left(D_{1}^{(n)} G\right)^{\Delta} \\
\downarrow p_{n} G^{\Delta} & \downarrow p_{n}\left(D_{1}^{(n)} G\right)^{\Delta} \\
P_{n} G^{\Delta} \stackrel{P_{n} p(n)}{\longrightarrow} & P_{n}\left(D_{1}^{(n)} G\right)^{\Delta} .
\end{array}
$$

Since $D_{1}^{(n)} G$ is $n$-multiadditive, the right vertical map is a quasi-isomorphism by Lemma 3.4 and Lemma 2.11. Hence it suffices to show that the lower horizontal map is also a quasi-isomorphism. To do this, we first observe, by using the unnormalized model for $D_{1}$, that $\left(D_{1}^{(n)} G\right)^{\Delta}$ is naturally the total complex of an $n$-dimensional complex given by

$$
\left(k_{1}, \cdots, k_{n}\right) \mapsto\left(\perp_{2}^{\left(k_{1}\right)} \cdots \perp_{2}^{\left(k_{n}\right)} G\right)^{\Delta},
$$

where $\perp_{2}^{\left(k_{1}\right)} \ldots \perp_{2}^{\left(k_{n}\right)} G$ denotes the functor produced by applying $\perp_{2}^{\left(k_{i}\right)}$ in the $i$ th variable. At degree $(0,0, \ldots, 0)$, the functor in this complex is $G^{\Delta}$ and $p(n)$ is the inclusion of $G^{\Delta}$ at this level. Hence $P_{n} p(n)$ is the inclusion of $P_{n} G^{\Delta}$ into the complex given by

$$
\left(k_{1}, \ldots, k_{n}\right) \mapsto P_{n}\left(\left(\perp_{2}^{\left(k_{1}\right)} \perp_{2}^{\left(k_{2}\right)} \ldots \perp_{2}^{\left(k_{n}\right)} G\right)^{\Delta}\right) .
$$

However, $P_{n}\left(\left(\perp_{2}^{\left(k_{1}\right)} \perp_{2}^{\left(k_{2}\right)} \ldots \perp_{2}^{\left(k_{n}\right)} G\right)^{\Delta}\right)$ is contractible when $k_{1}, \ldots, k_{n}$ are not all 0 . This follows from Lemma 3.3 once we recognize that $\left(\perp_{2}^{\left(k_{1}\right)} \perp_{2}^{\left(k_{2}\right)} \ldots \perp_{2}^{\left(k_{n}\right)} G\right)^{\Delta}$ is the diagonal of a functor of at least $n+1$ variables when $\left(k_{1}, \ldots, k_{n}\right) \neq(0, \ldots, 0)$, and is reduced in each of these variables. Thus $P_{n} p(n)$ is a quasi-isomorphism, and the result follows.

The previous results show that $D_{1}^{(n)} c r_{n} F^{\Delta}$ has certain properties in common with $D_{n} F$ : Lemma 3.4 implies that $D_{1}^{(n)} c r_{n} F^{\Delta}$ is degree $n$, and Proposition 3.2 implies that $P_{k}\left(D_{1}^{(n)} c r_{n} F^{\Delta}\right) \simeq 0$ for $k<n$. It remains to show that $D_{n} F$ is naturally equivalent to $\left(D_{1}^{(n)} c r_{n} F\right)_{h \Sigma_{n}}$, where $h \Sigma_{n}$ denotes the homotopy orbits with respect to the $n$th symmetric group action that permutes the variables of $c r_{n} F$. We review some of the properties of homotopy orbits that will be needed. 
Definition 3.5. Let $G$ be a group and $X$ a (left) $G$-object in $\mathcal{A}$. By the homotopy orbits of $X$ we mean the simplicial object in $\mathcal{A}$, denoted $X_{h G}$, given by

$$
\begin{gathered}
{[n] \mapsto G^{\times n} \times X,} \\
d_{i}\left(g_{1}, \ldots, g_{n}, x\right)= \begin{cases}\left(g_{2}, \ldots, g_{n}, x\right), & i=0, \\
\left(g_{1}, \ldots, g_{i} g_{i+1}, \ldots, g_{n}, x\right), & 1 \leq i \leq n-1, \\
\left(g_{1}, \ldots, g_{n-1}, g_{n} x\right), & i=n,\end{cases} \\
s_{j}\left(g_{1}, \ldots, g_{n}, x\right)=\left(g_{1}, \ldots, g_{j}, e, g_{j+1}, \ldots, g_{n}, x\right) .
\end{gathered}
$$

This can be written equivalently as $\mathbf{Z}[E G] \otimes_{\mathbf{Z}[G]} X$, where $E G$ is a free $G$-resolution of $\mathbf{Z}$. If $X_{*}$ is a chain complex of $G$-objects, then by $\left(X_{*}\right)_{h G}$ we mean the total complex of the natural complex obtained by applying ()$_{h G}$ degreewise.

When $\mathcal{A}$ is the category of abelian groups, $X$ is a $G$-module and the homology of $X_{h G}$ is the usual group homology of $G$ with coefficients in $X$. If $X$ is a free $G$-object (or a chain complex of free $G$-objects), then it follows from the definition above that $X_{h G} \simeq X_{G}$, where $X_{G}$ denotes the orbits of $X$ under the action of $G$. In particular, if $X \simeq \mathbf{Z}[G] \otimes_{\mathbf{Z}} Y$, then $X_{h G} \simeq X_{G} \simeq Y$. In general, orbits and homotopy orbits are distinct. For example, it is straightforward to show that homotopy orbits preserve quasi-isomorphisms, whereas the example below shows that orbits do not possess this property.

Example 3.6. Let $X$ be a $G$-object in $\mathcal{A}$, and let $P_{*} \stackrel{\simeq}{\longrightarrow} X$ be a resolution of $X$ by free $G$-objects. Orbits preserve this equivalence only when $H_{i}\left(X_{h G}\right)=0$ for $i>0$. To see this, apply the homotopy orbit construction degreewise to $P_{*}$ and $X$ to obtain a quasi-isomorphism of bicomplexes

$$
\left(P_{*}\right)_{h G} \stackrel{\simeq}{\longrightarrow} X_{h G},
$$

where $X_{h G}$ is a bicomplex with one nontrivial row. Note that since $P_{*}$ is composed of free $G$-objects, $P_{h G}$ is the total complex of a bicomplex in which the $i$ th row (in the direction of the homotopy orbit construction) is quasi-isomorphic to $\left(P_{i}\right)_{G}$. It follows that $\left(P_{*}\right)_{G} \simeq\left(P_{*}\right)_{h G} \simeq X_{h G}$. Moreover, $H_{0}\left(\left(P_{*}\right)_{G}\right) \cong X_{G}$. Hence $\left(P_{*}\right)_{G}$ is quasi-isomorphic to $X_{G}$ if and only if the homology of $X_{h G}$ vanishes in degrees greater than 0. But often this is not the case. For example, the homology of the $n$th symmetric group with coefficients in $\mathbf{Z}$ does not vanish in degrees greater than 0 .

To show that $D_{n} F$ is quasi-isomorphic to $D_{1}^{(n)} c r_{n} F_{h \Sigma_{n}}$ in the case where $F$ is degree $n$, we construct a natural transformation $\eta_{n}: c r_{n} F_{h \Sigma_{n}} \rightarrow F$, as follows. Since $H_{0}\left(X_{h G}\right)=X_{G}$, we have a natural sequence

$$
X \rightarrow X_{h G} \rightarrow X_{G}
$$

that factors the map $X \rightarrow X_{G}$. We will be interested in this map in the case where $X$ is the $n$th cross effect of a functor and $G$ is the $n$th symmetric group. Observe that the natural transformation $+: c r_{n} F \rightarrow F$ of Example 1.7 factors as

$$
c r_{n} F \rightarrow\left(c r_{n} F\right)_{\Sigma_{n}} \stackrel{+}{\longrightarrow} F,
$$

since $\bigvee_{n} X \stackrel{+}{\longrightarrow} X$ is $\Sigma_{n}$-invariant for any object $X$ in $\mathcal{C}$ when $\Sigma_{n}$ acts by permuting the terms of $\bigvee_{n} X$. We will consider the natural transformation

$$
c r_{n} F_{h \Sigma_{n}} \stackrel{\eta_{n}}{\longrightarrow} F
$$


produced by composing the map $\left(c r_{n} F\right)_{h \Sigma_{n}} \rightarrow\left(c r_{n} F\right)_{\Sigma_{n}}$ with + , and show that $\operatorname{cr}_{n}\left(\eta_{n}\right)$ is an equivalence for degree $n$ functors.

Lemma 3.7. If $F: \mathcal{C} \rightarrow C h \mathcal{A}$ is a degree $n$ functor, then $\mathrm{cr}_{n}\left(\eta_{n}\right)$ is a quasiisomorphism.

Before proving this lemma, note that ()$_{h G}$ is an additive functor on $\mathcal{A}$. As a result, when ()$_{h G}$ is applied to functors it commutes with cross effects and preserves the degree of a functor.

Proof. We begin by determining $c r_{n}\left(c r_{n} F\right)$. Since $F$ is degree $n$, we know that $c r_{n} F$ is additive in each of its variables. Thus, for objects $X_{1}, X_{2}, \ldots, X_{n}$ in $\mathcal{C}$,

$$
c r_{n} F\left(\bigvee_{i=1}^{t} X_{i}, \ldots, \bigvee_{i=1}^{t} X_{i}\right) \cong \bigoplus_{\beta \in \operatorname{Hom}(\langle n\rangle,\langle t\rangle)} c r_{n} F\left(X_{\beta(1)}, X_{\beta(2)}, \ldots, X_{\beta(n)}\right) .
$$

This is a $\Sigma_{n}$-equivariant isomorphism with the $\Sigma_{n}$-action on the right side given by $\sigma *(\beta, u)=\left(\beta \circ \sigma^{-1}, \sigma * u\right)$. It follows that

$$
c r_{n}\left(c r_{n} F\right) \cong \bigoplus_{\beta \in \Sigma_{n}} c r_{n} F \cong \mathbf{Z}\left[\Sigma_{n}\right] \otimes \mathbf{z} c r_{n} F .
$$

Under this identification, the $\Sigma_{n}$-action on $\mathbf{Z}\left[\Sigma_{n}\right] \otimes \mathbf{z} c r_{n} F$ is still given by

$$
\sigma *(\beta \otimes u)=\left(\beta \circ \sigma^{-1}\right) \otimes(\sigma * u)
$$

for $\beta \in \Sigma_{n}$ and $u \in c r_{n} F(X)$. Thus $c r_{n}\left(c r_{n} F\right)$ is a free $\Sigma_{n}$-object, and we obtain the indicated equivalences in the natural commutative diagram

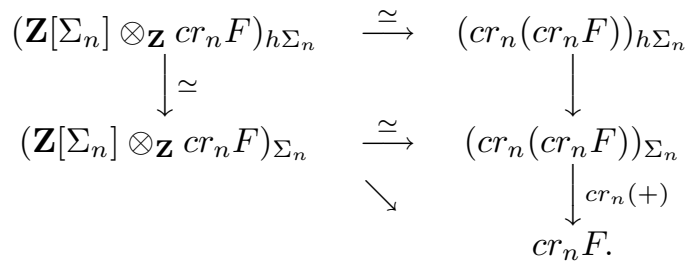

We claim that the composite from $\left(\mathbf{Z}\left[\Sigma_{n}\right] \otimes \mathbf{Z} c r_{n} F\right)_{\Sigma_{n}}$ to $c r_{n} F$ that factors through $\left(c r_{n}\left(c r_{n} F\right)\right)_{\Sigma_{n}}$ is a quasi-isomorphism. One can see this by observing that the composite

$$
\mathbf{Z}\left[\Sigma_{n}\right] \otimes \mathbf{z} c r_{n} F \stackrel{\simeq}{\longrightarrow} c r_{n}\left(c r_{n} F\right) \stackrel{c r_{n}(+)}{\longrightarrow} c r_{n} F
$$

is defined for $\beta \in \Sigma_{n}$ and $u \in c r_{n} F(X)$ by $\beta \otimes u \mapsto \beta * u$. This map is $\Sigma_{n}$-invariant under the $\Sigma_{n}$-action described above, so it induces the composite

$$
\left(\mathbf{Z}\left[\Sigma_{n}\right] \otimes \mathbf{Z} c r_{n} F\right)_{\Sigma_{n}} \stackrel{\simeq}{\longrightarrow}\left(c r_{n}\left(c r_{n} F\right)\right)_{\Sigma_{n}} \stackrel{c r_{n}(+)}{\longrightarrow} c r_{n} F .
$$

But this is naturally equivalent to the equivalence

$$
\left(\mathbf{Z}\left[\Sigma_{n}\right] \otimes \mathbf{z} c r_{n} F\right)_{\Sigma_{n}} \rightarrow c r_{n} F
$$

for free $\Sigma_{n}$-objects described after Definition 3.5. Hence the composite is an equivalence. It follows that the composition of the right vertical maps in the diagram is a quasi-isomorphism. Since this is $c r_{n}\left(\eta_{n}\right)$, the proof is complete.

Hence, when $F$ is degree $n, c r_{n}\left(c r_{n} F_{h \Sigma_{n}}\right) \simeq c r_{n} F$. In this case, $c r_{n} F$ and consequently $c r_{n}\left(c r_{n} F_{h \Sigma_{n}}\right)$ are additive in each variable. Thus, $c r_{n} F_{h \Sigma_{n}}$ is degree $n$ as well. We show below that $c r_{n} F_{h \Sigma_{n}}$ is quasi-isomorphic to $D_{n} F$. 
Lemma 3.8. If $F: \mathcal{C} \rightarrow C h \mathcal{A}$ is degree $n$, then $D_{n} F \simeq c r_{n} F_{h \Sigma_{n}}$.

Proof. Consider the following commutative diagram of chain complexes:

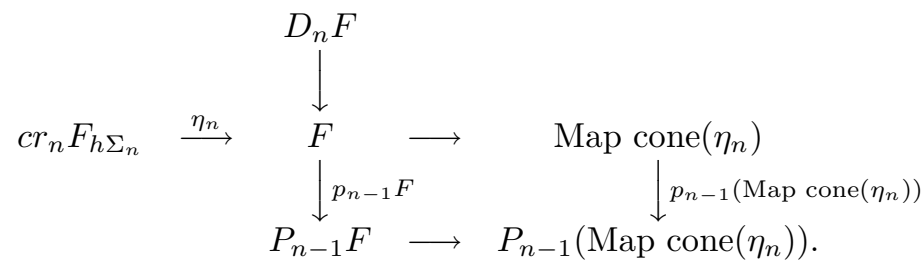

To conclude that $D_{n} F \simeq c r_{n} F_{h \Sigma_{n}}$, it suffices to show that the lower horizontal and rightmost vertical maps are equivalences. To do so, note that Lemma 3.7 implies that $c r_{n}$ (Map cone $\left.\left(\eta_{n}\right)\right) \simeq 0$, and so (Map cone $\left(\eta_{n}\right)$ ) is a degree $n-1$ functor. Thus, $p_{n-1}$ (Map cone $\left.\left(\eta_{n}\right)\right)$ is an equivalence. Now consider the exact sequence associated to the mapping cone:

$$
c r_{n} F_{h \Sigma_{n}} \rightarrow F \rightarrow \text { Map cone }\left(\eta_{n}\right) .
$$

By Proposition 3.2, $P_{n-1} c r_{n} F_{h \Sigma_{n}} \simeq 0$, and so applying $P_{n-1}$ to this exact sequence yields the desired equivalence from $P_{n-1} F$ to $P_{n-1}$ (Map cone $\left(\eta_{n}\right)$ ).

Using Lemma 3.8, we can describe $D_{n} F$ for any $F$.

Proposition 3.9. For any functor $F: \mathcal{C} \rightarrow C h \mathcal{A}, D_{n} F$ is naturally equivalent to $D_{1}^{(n)} c r_{n} F_{h \Sigma_{n}}$.

Proof. We start by showing that $D_{n} F \simeq D_{n} P_{n} F$, and then prove the result for $D_{n} P_{n} F$. Recall from Example 2.14.6 that $P_{n-1} P_{n} F \simeq P_{n-1} F$ via the natural transformation $P_{n-1}\left(p_{n}\right)$. To see that $D_{n} F \simeq D_{n} P_{n} F$, apply the five lemma to the long exact sequence in homology associated to the commutative diagram

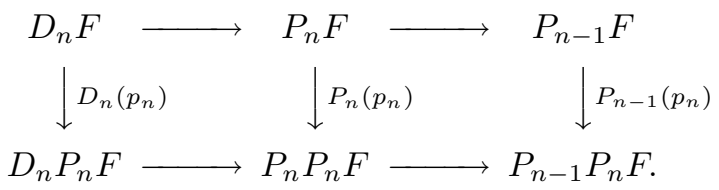

Hence, by Lemma 3.8, it suffices to show that $\mathrm{cr}_{n} P_{n} F_{h \Sigma_{n}}$ is naturally equivalent to $D_{1}^{(n)} c r_{n} F_{h \Sigma_{n}}$. However, $D_{1}^{(n)} c r_{n} F$ is $n$-multiadditive, and so by Lemma 3.4 its diagonal is degree $n$. Since homotopy orbits preserve degree, we have a natural quasi-isomorphism

$$
\left(D_{1}^{(n)} c r_{n} F\right)_{h \Sigma_{n}} \stackrel{\simeq}{\longrightarrow} P_{n}\left[D_{1}^{(n)} c r_{n} F_{h \Sigma_{n}}\right] .
$$

We claim that $c r_{n} P_{n} F_{h \Sigma_{n}}$ is also naturally equivalent to $P_{n}\left[D_{1}^{(n)} c r_{n} F_{h \Sigma_{n}}\right]$. To see this, consider the natural map $c r_{n} F \rightarrow D_{1}^{(n)} c r_{n} F$. This map is $\Sigma_{n}$-equivariant. Since $c r_{n} F$ is $n$-multireduced, the proof of Proposition 3.2 yields

$$
P_{n}\left(c r_{n} F^{\Delta}\right) \simeq P_{n}\left(D_{1}^{(n)} c r_{n} F^{\Delta}\right) .
$$

Hence $\left[P_{n}\left(c r_{n} F\right)\right]_{h \Sigma_{n}} \simeq\left[P_{n}\left(D_{1}^{(n)} c r_{n} F\right)\right]_{h \Sigma_{n}}$. Since cross effects commute (Remark $1.5)$ and homotopy orbits commute with the $P_{n}$ construction, it follows that

$$
c r_{n} P_{n} F_{h \Sigma_{n}} \simeq P_{n}\left(D_{1}^{(n)} c r_{n} F_{h \Sigma_{n}}\right) .
$$


Hence

$$
D_{n} F \simeq c r_{n} P_{n} F_{h \Sigma_{n}} \simeq P_{n}\left(D_{1}^{(n)} c r_{n} F_{h \Sigma_{n}}\right) \simeq\left(D_{1}^{(n)} c r_{n} F\right)_{h \Sigma_{n}}
$$

as desired.

We conclude with a remark to explain why homotopy orbits appear in the layers of the tower.

Remark 3.10. Suppose $F$ is degree $n$. Recall that $D_{n} F$ is the mapping cone of $P_{n} F \stackrel{q}{\longrightarrow} P_{n-1} F$. That is, it is Tot (shifted) of the following complex:

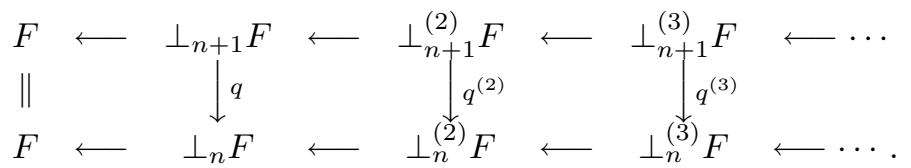

Since $F$ is degree $n$, it follows that $\perp_{n+1} F \simeq 0$, and hence we see that the natural inclusion of the bicomplex

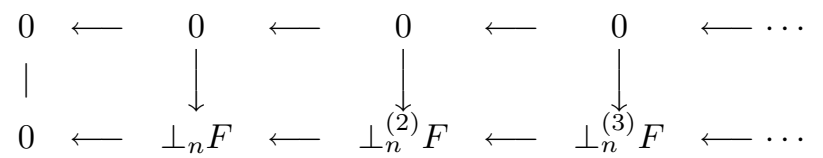

into $\left(D_{n}\right)$ is a quasi-isomorphism. Since $F$ is degree $n$, it follows that $\perp_{n} F$ is the diagonal of an $n$-multilinear functor, and hence we have a natural $\Sigma_{n}$-equivariant quasi-isomorphism, as in the proof of Lemma 3.7,

$$
\mathbf{Z}\left[\Sigma_{n}\right] \otimes \mathbf{z} \perp_{n} F \stackrel{\simeq}{\longrightarrow} \perp_{n}\left(\perp_{n} F\right)=\perp_{n}^{(2)} F .
$$

More generally, we obtain a natural isomorphism

$$
\mathbf{Z}\left[\Sigma_{n}^{\times(t+1)}\right] \otimes_{\mathbf{Z}\left[\Sigma_{n}\right]} \perp_{n} F \cong \mathbf{Z}\left[\Sigma_{n}^{\times(t)}\right] \otimes_{\mathbf{Z}} \perp_{n} F \stackrel{\simeq}{\longrightarrow} \perp_{n}^{(t+1)} F .
$$

If one tracks through these natural quasi-isomorphisms (we leave this to the interested reader), one sees that there is a map of complexes

$$
\mathbf{Z}\left[E \Sigma_{n}\right] \otimes_{\mathbf{Z}\left[\Sigma_{n}\right]} \perp_{n} F \stackrel{\simeq}{\longrightarrow} \perp_{n}^{(*)} F
$$

and hence a rather direct computation that, for $F$ of degree $n$,

$$
D_{n} F \simeq\left(\perp_{n} F\right)_{h \Sigma_{n}}=\left(c r_{n} F\right)_{h \Sigma_{n}} .
$$

Of course, since $c r_{n} F$ is already $n$-multilinear in this situation, we recover our previous characterization. Even when $F$ is strictly degree $n$, the layer $D_{n} F$ is still quasi-isomorphic to $\left(c r_{n} F\right)_{h \Sigma_{n}}$, and in this situation the inclusion of $\left(\perp_{n}\right)$ into $\left(D_{n}\right)$ is practically an isomorphism and $\left(\Sigma_{n}\right)$ is an isomorphism of complexes.

\section{Convergence and Continuation}

In this section, we examine the homotopy inverse limit of the Taylor tower

$$
\cdots \rightarrow P_{n} F \rightarrow P_{n-1} F \rightarrow \cdots \rightarrow P_{0} F
$$

constructed in Theorem 2.12, and its relationship to $F$. In particular, we are interested in determining conditions under which the tower converges to the original functor. We also prove a continuation principle for these limits in the following sense. We show that, under certain conditions, equivalence between the $P_{1}$ terms of the towers for two functors guarantees equivalence of the limits of the towers of the functors. In this section, the category $\mathcal{C}$ is a basepointed category with 
finite coproducts and $\mathcal{A}$ is a cocomplete abelian category that satisfies $\mathrm{AB} 4^{*}: \mathcal{A}$ is complete and the product of any set of surjections is a surjection.

To define the limit of the Taylor tower of Theorem 2.12 we use homotopy limits. We do this to ensure that the limit of the tower will preserve quasi-isomorphisms of functors. One method for constructing the homotopy limit of a sequence of chain maps is to replace the sequence $\left\{X_{n} \stackrel{f_{n}}{\longrightarrow} X_{n-1}\right\}_{n \in \mathbf{N}}$ in $C h \mathcal{A}$ by a sequence $\left\{X_{n}^{\prime} \stackrel{f_{n}^{\prime}}{\longrightarrow} X_{n-1}^{\prime}\right\}_{n \in \mathbf{N}}$ in $C h \mathcal{A}$ in which each $f_{n}^{\prime}$ is a surjection and $X_{n}^{\prime}$ is quasiisomorphic to $X_{n}$ for each $n$. This can be done as follows.

Remark 4.1. For a chain complex $Y$, let $C(Y)$ denote the cone on $Y$, i.e., $C(Y)=$ Map cone $\left(1_{Y}\right)$. Let $C(Y)[-1]$ be the chain complex obtained by shifting $C(Y)$ down one degree. In this way, we have $C(Y)[-1]_{n}=C(Y)_{n+1}=Y_{n+1} \oplus Y_{n}$, and so the chain map $C(Y) \rightarrow Y[+1]$ shifts down to a chain map $y: C(Y)[-1] \rightarrow Y$. Given a chain map $X \stackrel{f}{\longrightarrow} Y$, we can form the commutative diagram

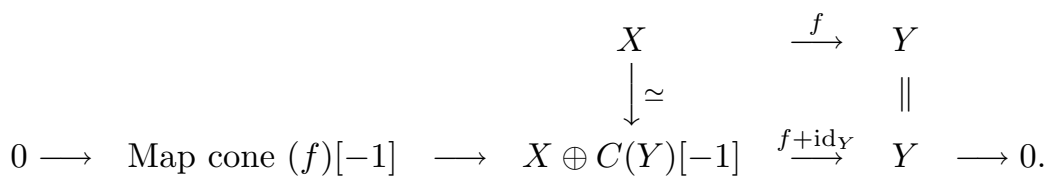

From this we see that any chain map $f$ can be replaced by a surjective chain map using a natural diagram of quasi-isomorphisms in which the kernel of the new map is the homotopy fiber of $f$. Moreover, it follows that any sequence of chain maps $\left\{X_{n} \stackrel{f_{n}}{\longrightarrow} X_{n-1}\right\}_{n \in \mathbf{N}}$ can be naturally replaced with a sequence of surjective chain maps $\left\{X_{n}^{\prime} \stackrel{f_{n}^{\prime}}{\longrightarrow} X_{n-1}^{\prime}\right\}_{n \in \mathbf{N}}$ such that the new chain maps are related to the original maps by commuting lattices of quasi-isomorphisms

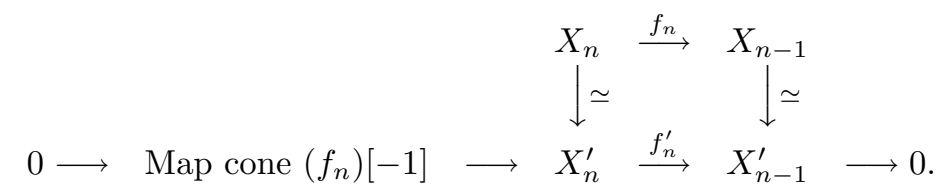

Using the new sequence of chain complexes $\left\{X_{n}^{\prime} \stackrel{f_{n}^{\prime}}{\longrightarrow} X_{n-1}^{\prime}\right\}_{n \in \mathbf{N}}$, we define the homotopy limit.

Definition 4.2. Let $\left\{X_{n}^{\prime} \stackrel{f_{n}^{\prime}}{\longrightarrow} X_{n-1}^{\prime}\right\}_{n \in \mathbf{N}}$ be the sequence of surjections obtained from a sequence $\left\{X_{n} \stackrel{f_{n}}{\longrightarrow} X_{n-1}\right\}_{n \in \mathbf{N}}$ as in Remark 4.1. The homotopy inverse limit (or homotopy limit) of $\left\{X_{n} \stackrel{f_{n}}{\longrightarrow} X_{n-1}\right\}_{n \in \mathbf{N}}$ is given by

$$
\operatorname{holim}_{n} X_{n}=\lim _{n} X_{n}^{\prime} \text {. }
$$

Note that this definition is functorial. Replacing chain maps of a sequence $\left\{X_{n} \stackrel{f_{n}}{\longrightarrow} X_{n-1}\right\}_{n \in \mathbf{N}}$ in $C h \mathcal{A}$ by surjections has the advantage that the resulting sequence $\left\{X_{n}^{\prime} \stackrel{f_{n}^{\prime}}{\longrightarrow} X_{n-1}^{\prime}\right\}$ satisfies the Mittag-Leffler condition, and so we obtain from it a short exact sequence (see We], pp. 82-83)

$$
0 \longrightarrow \lim _{n}^{1} H_{i+1}\left(X_{n}^{\prime}\right) \longrightarrow H_{i}\left(\lim _{n} X_{n}^{\prime}\right) \longrightarrow \lim _{n} H_{i}\left(X_{n}^{\prime}\right) \longrightarrow 0 .
$$

With the definition of homotopy limit this exact sequence becomes

$$
0 \longrightarrow \lim _{n}^{1} H_{i+1}\left(X_{n}\right) \longrightarrow H_{i}\left(\operatorname{holim}_{n} X_{n}\right) \longrightarrow \lim _{n} H_{i}\left(X_{n}\right) \longrightarrow 0 .
$$


From this sequence, we see that if each $X_{n} \in C h_{\geq 0} \mathcal{A}$, then the homology of holim ${ }_{n} X_{n}$ is concentrated in degrees $\geq-1$. If each $f_{n}$ is a surjection on $H_{0}$, then the homology of holim ${ }_{n} X_{n}$ is concentrated in non-negative degrees. Note however that the chain complex holim ${ }_{n} X_{n}$ will not necessarily be bounded below, because of the mapping cone used to construct each $X_{n}^{\prime}$.

With homotopy limits, we are able to discuss convergence of the sequence $\left\{P_{n} F\right.$ $\left.\stackrel{q_{n} F}{\longrightarrow} P_{n-1} F\right\}$.

Definition 4.4. Let $F$ be a functor from $\mathcal{C}$ to $C h \mathcal{A}$.

a) The limit of the tower

$$
\ldots \stackrel{q_{n+1} F}{\longrightarrow} P_{n} F(X) \stackrel{q_{n} F}{\longrightarrow} P_{n-1} F(X) \stackrel{q_{n-1} F}{\longrightarrow} \ldots \longrightarrow P_{0} F
$$

is the functor $P_{\infty} F=\operatorname{holim}_{n} P_{n} F$. There is a natural transformation $p_{\infty} F: F \longrightarrow P_{\infty} F$ that comes from the composite maps $p_{n} F: F \longrightarrow$ $P_{n} F \stackrel{\simeq}{\longrightarrow} P_{n} F^{\prime}$

b) We say that the tower

$$
\ldots \stackrel{q_{n+1} F}{\longrightarrow} P_{n} F(X) \stackrel{q_{n} F}{\longrightarrow} P_{n-1} F(X) \stackrel{q_{n-1} F}{\longrightarrow} \ldots \longrightarrow P_{0} F
$$

converges for the object $X$ provided that $F(X) \stackrel{p_{\infty} F(X)}{\longrightarrow} P_{\infty} F(X)$ is a quasiisomorphism.

From this definition and results about the functors $P_{n} F$, we have the following.

Lemma 4.5. a) If $F$ is a degree $n$ functor from $\mathcal{C}$ to $C h \mathcal{A}$, then $p_{\infty} F$ is an equivalence. That is, the tower converges for all objects $X$ in $\mathcal{C}$.

b) The functor $P_{\infty}$ preserves quasi-isomorphisms and short exact sequences of functors.

Proof. To see that a) is true, let $F$ be a degree $n$ functor. Then, for any $i$ we have $H_{i}\left(P_{t} F\right) \cong H_{i}\left(P_{t-1} F\right)$ for any $t>n$, and so the sequence

$$
\cdots \rightarrow H_{i}\left(P_{t} F\right) \rightarrow H_{i}\left(P_{t-1} F\right) \rightarrow \cdots \rightarrow H_{i}\left(P_{0} F\right)
$$

satisfies the Mittag-Leffler condition. Hence $\lim ^{1} H_{i+1}\left(P_{t} F\right) \cong 0$, and the result follows by using the sequence (4.3).

To see that b) is true, let $F \rightarrow G \rightarrow H$ be a short exact sequence of functors. It is straightforward to verify that $P_{n} F^{\prime} \rightarrow P_{n} G^{\prime} \rightarrow P_{n} H^{\prime}$ is also a short exact sequence of functors. Since the sequences $\left\{P_{n} F^{\prime}\right\},\left\{P_{n} G^{\prime}\right\}$, and $\left\{P_{n} H^{\prime}\right\}$ satisfy the Mittag-Leffler condition,

$$
\lim _{n}^{1} P_{n} F^{\prime} \cong \lim _{n}^{1} P_{n} G^{\prime} \cong \lim _{n}^{1} P_{n} H^{\prime} \cong 0 .
$$

Hence, since lim is left exact, the sequence

$$
0 \rightarrow \lim _{n} P_{n} F^{\prime} \rightarrow \lim _{n} P_{n} G^{\prime} \rightarrow \lim _{n} P_{n} H^{\prime} \rightarrow 0
$$

is exact and $P_{\infty}$ preserves exact sequences. The fact that $P_{\infty}$ preserves quasiisomorphisms follows from this by using the exact sequence associated to the mapping cone of the quasi-isomorphism, and showing that $P_{\infty}$ preserves acyclicity.

We can also establish the following result. This will be used in our discussion of continuation later in the section. 
Lemma 4.6. For any functor $F: \mathcal{C} \longrightarrow C h \mathcal{A}$, the natural transformation

$$
P_{k}\left(p_{\infty} F\right): P_{k} F \longrightarrow P_{k} P_{\infty} F
$$

is a quasi-isomorphism for all objects in $\mathcal{C}$.

Proof. We prove this result by first showing that $P_{k} P_{\infty} F \simeq \operatorname{holim}_{n}\left(P_{k} P_{n} F\right)$ and then using (4.3). From Definition 4.2 one can show that

$$
\operatorname{holim}_{n} X_{n} \simeq \text { fiber }\left(\prod_{i \in \mathbb{N}} X_{i} \stackrel{\omega}{\longrightarrow} \prod_{\alpha: X_{j} \rightarrow X_{i}} X_{i}\right),
$$

where $\omega$ is the difference of the maps

$$
\begin{aligned}
& \omega_{1}:\left(x_{i}\right)_{i \in \mathbb{N}} \mapsto\left(x_{i}\right)_{\alpha: X_{j} \rightarrow X_{i}}, \\
& \omega_{2}:\left(x_{i}\right)_{i \in \mathbb{N}} \mapsto\left(\alpha\left(x_{i}\right)\right)_{\alpha: X_{i} \rightarrow X_{j}} .
\end{aligned}
$$

One then has the following sequence of equivalences by using the above and noting that the conditions placed on $\mathcal{A}$, in particular $\mathrm{AB} 4^{*}$, guarantee that $P_{n}$ commutes with arbitrary products of functors:

$$
\begin{aligned}
P_{k}\left(\operatorname{holim}_{n} P_{n} F\right) & \simeq P_{k} \text { fiber }\left[\prod_{i \in \mathbb{N}} P_{i} F \stackrel{\omega}{\longrightarrow} \prod_{\alpha: P_{j} F \rightarrow P_{i} F} P_{i} F\right] \\
& \simeq \operatorname{fiber}\left(P_{k} \prod_{i \in \mathbb{N}} P_{i} F \stackrel{P_{k} \omega}{\longrightarrow} P_{k} \prod_{\alpha: P_{j} F \rightarrow P_{i} F} P_{i} F\right) \\
& \simeq \operatorname{fiber}\left(\prod_{i \in \mathbb{N}} P_{k} P_{i} F \longrightarrow \prod_{\alpha: P_{j} F \rightarrow P_{i} F} P_{k} P_{i} F\right) \\
& \simeq \operatorname{holim}_{n} P_{k} P_{n} F .
\end{aligned}
$$

Thus $P_{k} P_{\infty} F \simeq \operatorname{holim}_{n} P_{k} P_{n} F$. Applying (4.3) to holim $P_{k} P_{n} F$, we have

$$
0 \longrightarrow \lim _{n}^{1} H_{i+1}\left(P_{k} P_{n} F\right) \longrightarrow H_{i}\left(\operatorname{holim}_{n} P_{k} P_{n} F\right) \longrightarrow \lim _{n} H_{i}\left(P_{k} P_{n} F\right) \longrightarrow 0 .
$$

From this sequence we see that $\operatorname{holim}_{n} P_{k} P_{n} F \simeq P_{n} F$, after recalling that $P_{k} P_{n} F \simeq$ $P_{n} F$ (Example 2.14.6) for $k<n$. The result follows.

We next describe the extent to which the functors $F$ and $P_{n} F$ agree, and conditions under which $F$ and $P_{\infty} F$ agree. To do so, we need a way to compare chain complexes and functors to chain complexes. Our method of comparison is given in the following definition.

Definition 4.7. The chain complex $A$ is $n$-connected if $H_{*}(A)=0$ for $* \leq n$. A chain map $f: A \longrightarrow B$ is $n$-connected if $H_{*}(f)$ is an isomorphism for $*<n$ and a surjection for $*=n$. That is, a chain map is $n$-connected if and only if its homotopy fiber is $(n-1)$-connected.

By placing a connectivity condition on the iterated cross effects of a functor, we can guarantee convergence of the Taylor tower.

Proposition 4.8. Let $F: \mathcal{C} \longrightarrow C h \mathcal{A}$, and let $X$ be an object in $\mathcal{C}$. If there is a constant $c$ such that the connectivity of $\perp_{n+1}^{(t)} F(X)$ is at least $c-(t-1)$ for any $1 \leq t \leq c$, then $p_{n} F: F(X) \longrightarrow P_{n} F(X)$ is c-connected. 
Proof. The homotopy fiber of $F \stackrel{p_{n} F}{\longrightarrow} P_{n} F$ is quasi-isomorphic to the chain complex obtained from the simplicial object $\perp_{n+1}^{*+1} F$ defined in Remark 2.3 for the cotriple $\perp_{n+1}=\Delta^{*} \circ \mathrm{cr}_{n+1}$. When evaluated at $X, \perp_{n+1}^{*+1} F(X)$ is a bicomplex of objects in $\mathcal{A}$. Consider the spectral sequence associated to this bicomplex. Taking homology of this bicomplex in the direction of the $t^{\prime}$ s yields an $E^{1}$ sheet that by our initial assumption looks like

$\begin{array}{lcccccc}H_{*}\left(\perp^{c+1} F(X)\right) & \star & \star & & & & \\ H_{*}\left(\perp^{c} F(X)\right) & 0 & \star & & & & \\ \quad \vdots & \vdots & \vdots & \vdots & \vdots & \vdots & \vdots \\ H_{*}\left(\perp^{3} F(X)\right) & 0 & 0 & \cdots & \star & \star & \star \\ H_{*}\left(\perp^{2} F(X)\right) & 0 & 0 & \cdots & 0 & \star & \star \\ H_{*}(\perp F(X)) & 0 & 0 & \cdots & 0 & 0 & \star \\ \operatorname{dim} & 0 & 1 & \cdots & c-1 & c & c+1 .\end{array}$

Hence the homology of $\perp_{n+1}^{*+1} F$ is 0 in degrees $\leq c$, and so $p_{n} F(X)$ is $c$-connected.

With Proposition 4.8, we have a means of measuring whether or not the sequence $\ldots \longrightarrow P_{n} F \stackrel{q_{n} F}{\longrightarrow} P_{n-1} F \longrightarrow \ldots$ converges for a given object $X$.

Definition 4.9. Let $F: \mathcal{C} \longrightarrow C h \mathcal{A}$, and let $X$ be an object in $\mathcal{C}$. For $n \geq 0$ we set

$$
F_{\text {con }}(X, n)=\max \left\{c \in \mathbf{Z} \mid \operatorname{conn}\left(\perp_{n}^{(t)} F(X)\right) \geq c-(t-1) \text { for } 1 \leq t \leq c\right\} .
$$

Corollary 4.10. If $\lim _{n \rightarrow \infty} F_{\text {con }}(X, n)=N$, then $p_{\infty} F: F(X) \rightarrow P_{\infty} F(X)$ is $(N-1)$-connected. In particular, when $N=\infty$ the Taylor tower converges for $X$.

Proof. Let $M_{n} F(X)=\operatorname{fiber}\left(F(X) \stackrel{p_{n} F}{\longrightarrow} P_{n} F(X)\right)$. Consider the exact sequence derived from (4.3):

$$
0 \rightarrow \lim _{n}{ }^{1} H_{i+1}\left(M_{n} F(X)\right) \rightarrow H_{i}\left(\operatorname{holim}_{n}\left(M_{n} F(X)\right)\right) \rightarrow \lim _{n} H_{i}\left(M_{n} F(X)\right) \rightarrow 0 .
$$

By Proposition 4.8 and our connectivity assumption, $\lim _{n} H_{i}\left(M_{n} F(X)\right)=0$ and $\lim _{n}^{1} H_{i}\left(M_{n} F(X)\right)=0$ for all $i \leq N-1$. Hence, $H_{i}\left(\operatorname{holim}_{n}\left(M_{n} F(X)\right)\right.$ is $(N-2)$ connected. The result follows by noting that

$$
\operatorname{fiber}\left(F(X) \longrightarrow P_{\infty} F(X)\right) \cong \operatorname{holim}_{n}\left(F(X) \longrightarrow P_{n} F(X)\right) \text {. }
$$

Example 4.11. Let $F$ be the simplicial prolongation of a functor from an abelian category $\mathcal{A}$ to an abelian category $\mathcal{B}$. For a $k$-reduced chain complex $X$ of objects in $\mathcal{A}$, one can use the Eilenberg-Zilber theorem (see [J-M1, 7.1 and 3.6) to show that $\perp_{n} F(X)$ is $(n k+n-1)$-reduced. It follows that $F_{\text {con }}(X, n) \geq n k+n-1$ for all $n$. By Corollary 4.10, the Taylor tower of $F$ converges for all $k$-reduced $X$ with $k \geq 0$.

We now focus our attention on the continuation principle that we described at the beginning of this section. This result was inspired by one of the mostly widely-used results of Goodwillie's theory. In essence, the result allows one to establish that a natural transformation is an equivalence of functors by proving it is an equivalence 
on the first terms of the associated Taylor towers. To see how this type of result has been applied to $K$-theory, we refer the reader to [CCGH] and [M1]. Our result will be expressed in terms of the following functors and categories.

Definition 4.12. 1) Fix $X \in \mathcal{C}$. Let $\mathcal{C}_{X}=X \backslash \mathcal{C} / X$ be the over/under category of $\mathcal{C}$ with respect to $X$. That is, $\mathcal{C}_{X}$ is the basepointed category with finite coproducts whose objects are objects of the form $C \vee X$, where $C$ is an object in $\mathcal{C}$, together with structure maps $i_{C} \in \operatorname{Hom}_{\mathcal{C}}(X, C \vee X), \pi_{C} \in \operatorname{Hom}_{\mathcal{C}}(C \vee X, X)$ such that $\pi_{C} \circ i_{C}=1_{X}$. The morphism set of $\mathcal{C}_{X}$ consists of those morphisms from $\mathcal{C}$ that preserve the structure maps, i.e.,

$\operatorname{Hom}_{\mathcal{C}_{X}}\left(C \vee X, C^{\prime} \vee X\right)=\left\{\alpha \in \operatorname{Hom}_{\mathcal{C}}\left(C \vee X, C^{\prime} \vee X\right) \mid \alpha \circ i_{C}=i_{C^{\prime}}\right.$ and $\left.\pi_{C}=\pi_{C^{\prime}} \circ \alpha\right\}$.

2) If $F$ is a functor from $\mathcal{C}$ to $C h \mathcal{A}$, then $F_{X}$ is the reduced functor from $\mathcal{C}_{X}$ to $C h \mathcal{A}$ defined by

$$
F_{X}\left(C \vee X ; i_{C}, \pi_{C}\right)=\operatorname{kernel}\left[F(C \vee X) \stackrel{F\left(\pi_{C}\right)}{\longrightarrow} F(X)\right] .
$$

Lemma 4.13. Let $F_{X}: \mathcal{C}_{X} \rightarrow C h \mathcal{A}$ be derived from $F: \mathcal{C} \rightarrow C h \mathcal{A}$ as above.

a) For objects $X_{1} \vee X, X_{2} \vee X, \ldots, X_{n} \vee X$ in $\mathcal{C}_{X}$,

$c r_{n} F_{X}\left(X_{1} \vee X, X_{2} \vee X, \ldots, X_{n} \vee X\right) \cong c r_{n} F\left(X_{1}, \ldots, X_{n}\right) \oplus c r_{n+1} F\left(X, X_{1}, \ldots, X_{n}\right)$.

b) For any object $Y \vee X$ in $\mathcal{C}_{X},\left(F_{X}\right)_{Y \vee X} \cong F_{Y \vee X}$.

Proof. To prove a), note that for an object $Y \vee X$ in $\mathcal{C}_{X}$, it follows from the definitions of cross effects and $F_{X}$ that

$$
F_{X}(Y \vee X) \cong c r_{1} F(Y) \oplus c r_{2} F(X, Y) .
$$

Hence,

$$
c r_{2} F_{X}(Y \vee X, Z \vee X) \cong c r_{2} F(Z, Y) \oplus c r_{3} F(X, Y, Z) .
$$

The result follows by induction.

To see that b) is true, consider the following commutative diagram:

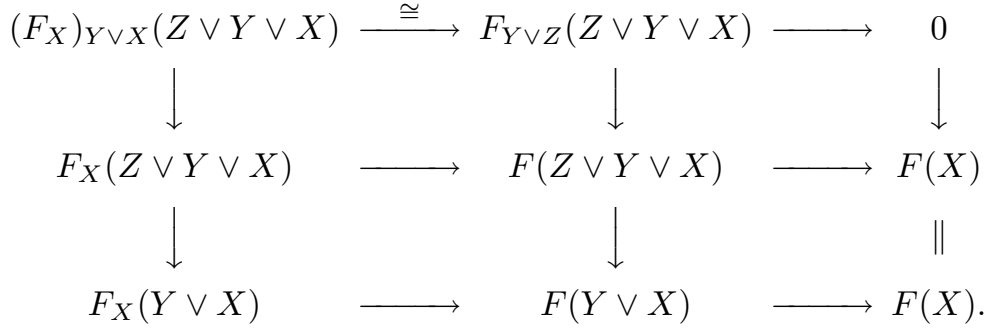

With this we can prove the desired continuation result.

Proposition 4.14. Let $\eta: F \longrightarrow G$ be a natural transformation of functors from $\mathcal{C}$ to $C h \mathcal{A}$. The following are equivalent.

a) $P_{1} \eta_{X}: P_{1} F_{X} \rightarrow P_{1} G_{X}$ is an equivalence for all $X \in \mathcal{C}$.

b) $P_{n} \eta_{X}: P_{n} F_{X} \rightarrow P_{n} G_{X}$ is an equivalence for all $n$ and all $X \in \mathcal{C}$.

c) $P_{\infty} \eta_{X}: P_{\infty} F_{X} \rightarrow P_{\infty} G_{X}$ is an equivalence for all $X \in \mathcal{C}$.

We prove Proposition 4.14 by first establishing the following special case.

Lemma 4.15. Let $F: \mathcal{C} \longrightarrow C h \mathcal{A}$. If $P_{1} F_{X} \simeq 0$ for all $X \in \mathcal{C}$, then $P_{n} F_{X} \simeq 0$ for all $n$ and $X \in \mathcal{C}$. 
Proof. Consider the exact sequence of functors $D_{n} F_{X} \rightarrow P_{n} F_{X} \rightarrow P_{n-1} F_{X}$. Using this sequence and induction, it suffices to show that $D_{n} F_{X} \simeq 0$ for all $n$ and $X$. By Proposition 3.9 we know that $D_{n} F_{X} \simeq\left(D_{1}^{(n)} c r_{n} F_{X}\right)_{h \Sigma_{n}}$. Thus it is enough to prove that $D_{1} c r_{n} F_{X}\left(-, X_{2}, \ldots, X_{n}\right) \simeq 0$ for all $X, X_{2}, \ldots, X_{n}$. We do this by first proving it in the case that $X=\star$, where $\star$ is the initial/final object in $\mathcal{C}$.

By our hypothesis, $D_{1} F_{X} \simeq P_{1} F_{X} \simeq 0$ for all $X$. But, by Lemma 4.13a and the fact that $D_{1}$ preserves direct sums of functors,

$0 \simeq D_{1} F_{X}(Y \vee X) \simeq D_{1} c r_{1} F(Y) \oplus D_{1} c r_{2} F(X, Y) \simeq D_{1} c r_{1} F_{\star}(Y) \oplus D_{1} c r_{2} F_{\star}(X, Y)$, for any $X$ and $Y$. Hence $D_{1} c r_{2} F_{\star} \simeq 0$. For $n \geq 2, D_{1} c r_{n} F_{\star}\left(X_{1}, X_{2}, \ldots, X_{n}\right)$ is a direct summand of $D_{1} c r_{n-1} F_{\star}\left(X_{1} \vee X_{2}, \ldots, X_{n}\right)$, and so by induction $D_{1} c r_{n} F_{\star} \simeq 0$ for all $n$. Now consider $D_{1} c r_{n} F_{X}$. By Lemma 4.13b, $F_{X} \simeq\left(F_{\star}\right)_{X}$. Hence, by Lemma 4.13a, $D_{1} c r_{n} F_{X} \simeq 0$, and the result follows.

Proof of Proposition 4.14. For $X \in \mathcal{C}$, let $M_{X}$ be the functor given by the mapping cone of $\eta_{X}$. By applying Lemma 4.15 to $M_{X}$ we see that a) and b) are equivalent. To see that b) and c) are equivalent, note that by Lemma $4.5 \mathrm{~b}, P_{\infty} \eta_{X}$ is an equivalence if and only if $P_{\infty} M_{X} \simeq 0$. Now assume c) is true. Then $P_{\infty} M_{X} \simeq 0$ and, by Lemma 4.6, $P_{n} M_{X} \simeq P_{n}\left(P_{\infty} M_{X}\right) \simeq 0$. Hence b) holds. Conversely, if b) is true, then $H_{i}\left(P_{n} M_{X}\right) \cong 0$ for all $i, n$ and $X$. Using (4.3), we see that $H_{i}\left(P_{\infty} M_{X}\right) \cong 0$, and so $P_{\infty} \eta_{X}$ is an equivalence.

\section{The Differential OF A FUNCTOR}

Our goal in this section is to define "differentials" for functors and determine how these differentials behave, especially with respect to the components of the Taylor tower. After motivating and stating the definition for differentials at the beginning of the section, we establish their basic properties, including a product rule and chain rule. We then examine how the differentials are related to the functor $D_{n}$. To complete the section, we determine the effect of the differentials on the functors $P_{n}$. In particular, we will show that $\nabla P_{n} \simeq P_{n-1} \nabla$, where $\nabla$ denotes the differential construction. We assume throughout this section that $\mathcal{C}$ is a basepointed category with finite coproducts, $\mathcal{A}$ is a cocomplete abelian category, and $F$ is a functor from $\mathcal{C}$ to $C h \mathcal{A}$.

Recall that for a differentiable multivariable function $f$, the derivative of $f$ at the point $y$ in the direction $v$ is determined by

$$
\nabla_{v} f(y)=\lim _{h \rightarrow 0} \frac{f(y+h v)-f(y)}{h} .
$$

This is a function of two variables, $v$ and $y$, that is linear with respect to the first variable, but not, in general, with respect to the second variable. The function $t \mapsto \nabla_{t v} f(y)$ determines the tangent line to the graph of $f$ in the direction $v$.

To mimic this construction for the differential of a functor, we begin by noting that

$$
F_{Y}(X)=\operatorname{ker}(F(Y \vee X) \rightarrow F(Y))
$$

plays the same role in our setting as the expression $f(y+v)-f(y)$. Moreover, applying $D_{1}$ to a functor produces the degree 1, reduced component of the functor, and hence corresponds to $\lim _{h \rightarrow 0} \frac{1}{h}$ in the expression above. Thus, we define $\nabla_{X} F(Y)$ for a functor $F$ as follows. 
Definition 5.1. Let $F: \mathcal{C} \rightarrow C h \mathcal{A}$, and let $X$ and $Y$ be objects in $\mathcal{C}$. The differential of $F$ is the bifunctor defined by

$$
\nabla_{X} F(Y)=\nabla F(X ; Y)=D_{1} F_{Y}(Y \vee X) .
$$

Remarks 5.2. a) Recall from the proof of Lemma 4.13 that $F_{Y}(Y \vee X) \cong c r_{2} F(X, Y)$ $\oplus c r_{1} F(X)$. Hence,

$$
\begin{aligned}
\nabla F(X ; Y) & =D_{1} F_{Y}(X \vee Y) \\
& =D_{1}\left[c r_{2} F(-, Y) \oplus c r_{1} F(-)\right](X) \\
& =D_{1}^{1} c r_{2} F(X, Y) \oplus D_{1} F(X),
\end{aligned}
$$

where $D_{1}^{1}$ denotes the linearization of the bifunctor $c r_{2} F$ with respect to its first variable. If we hold $Y$ fixed, it follows immediately that $\nabla F(-; Y)$ is degree 1 with respect to its first variable. In general, $\nabla F$ is not degree 1 with respect to its second variable, as $\operatorname{cr}_{2} F(X, Y)$ is degree one in its second variable if and only if $F$ is a degree two functor.

b) It follows from a) that the functor of functors $\nabla$ preserves short exact sequences. That is, given a short exact sequence of functors

$$
0 \rightarrow F^{\prime \prime} \stackrel{\alpha}{\longrightarrow} F \stackrel{\beta}{\longrightarrow} F^{\prime} \rightarrow 0
$$

then

$$
0 \rightarrow \nabla F^{\prime \prime} \stackrel{\nabla \alpha}{\longrightarrow} \nabla F \stackrel{\nabla \beta}{\longrightarrow} \nabla F^{\prime} \rightarrow 0
$$

is a short exact sequence of functors as well.

We wish to establish some properties of the differential that are analogous to properties of the differential for multivariable functions. The first property is essentially a restatement of Proposition 4.14 in terms of the differential, and is a version of Goodwillie's "first derivative criterion" ([G2], theorem 5.10). Roughly, it says that if the differentials of two functors agree in a neighborhood of the object $Y$, then the functors themselves agree up to a constant. More precisely, we have

Proposition 5.3. Let $F, G: \mathcal{C} \rightarrow C h \mathcal{A}$, and let $\eta: F \rightarrow G$ be a natural transformation. If $\nabla \eta(X ; Y)$ is an equivalence for all $X$ and $Y$, then $P_{\infty} \eta_{Y}: P_{\infty} F_{Y} \rightarrow P_{\infty} G_{Y}$ is an equivalence for all $Y$.

Proof. By definition, $\nabla F(-; Y)=D_{1} F_{Y}$. Hence $\nabla \eta(-; Y): D_{1} F_{Y} \stackrel{\simeq}{\longrightarrow} D_{1} G_{Y}$, and as a consequence $P_{1} F_{Y} \stackrel{\simeq}{\longrightarrow} P_{1} G_{Y}$. The result follows by Proposition 4.14.

The next property demonstrates that $\nabla$ lowers the degree of a functor by 1 .

Proposition 5.4. If $F: \mathcal{C} \rightarrow C h \mathcal{A}$ is a degree $n$ functor, then $\nabla F$ is degree $n-1$ with respect to its second variable.

Proof. By Remark 1.5,

$$
c r_{n}\left(c r_{2} F\left(X_{1},-\right)\right)\left(X_{2}, \ldots, X_{n+1}\right) \simeq c r_{2}\left(c r_{n} F\left(-, X_{3}, \ldots, X_{n+1}\right)\right)\left(X_{1}, X_{2}\right) .
$$

But since $F$ is degree $n, c r_{n} F$ is additive in each of its variables. Hence

$$
c r_{2}\left(c r_{n} F\left(-, X_{3}, \ldots, X_{n+1}\right)\right) \simeq 0 .
$$

It follows by Remark 5.2 a that $c r_{n} \nabla F(X ;-) \cong 0$, since $D_{1} F(X)$ is constant with respect to the second variable of $\nabla F$, and it is straightforward to show that $c r_{n} D_{1}^{1} c r_{2} F \simeq D_{1}^{1} c r_{n} c r_{2} F$. Therefore $\nabla F(X ;-)$ is degree $n-1$. 
We can also determine product and chain rules for the differential.

Proposition 5.5 (Product rule). Let $\mu$ be a biadditive functor from $C h \mathcal{A}$ to itself, i.e., $\mu$ is a functor of two variables that is degree one in each of its variables. Let $F$ and $G$ be reduced functors from $\mathcal{C}$ to $C h \mathcal{A}$. The bifunctor $\mu(F, G)$ can be treated as a functor from $\mathcal{C}$ by composing with the diagonal functor. When $\mu$ is considered as a functor of one variable in this way, we have

$$
\nabla \mu(F, G) \simeq \mu(\nabla F, G) \oplus \mu(F, \nabla G) .
$$

That is, for objects $X$ and $Y$ in $\mathcal{C}$,

$$
\nabla \mu(F, G)(X ; Y) \simeq \mu(\nabla F(X ; Y), G(Y)) \oplus \mu(F(Y), \nabla G(X ; Y)) .
$$

Proof. We first observe that since $\mu$ is biadditive, $\operatorname{cr}_{2} \mu(F, G)(X, Y)$ is equivalent to

$$
\begin{array}{ccccc} 
& & \mu(F(X), G(Y)) & \oplus & \mu\left(F(X), c r_{2} G(X, Y)\right) \\
\mu(F(Y), G(X)) & \oplus & & \oplus & \mu\left(F(Y), c r_{2} G(X, Y)\right) \\
\mu\left(c r_{2} F(X, Y), G(X)\right) & \oplus & \mu\left(c r_{2} F(X, Y), G(Y)\right) & \oplus & \mu\left(c r_{2} F(X, Y), c r_{2} G(X, Y)\right) .
\end{array}
$$

By Lemma 3.3, some of these terms vanish after applying $D_{1}$ in the first variable. In particular, we have

$$
\begin{aligned}
D_{1}\left[\mu\left(F(-), c r_{2} G(-, Y)\right)\right](X) & \simeq 0, \\
D_{1}\left[\mu\left(c r_{2} F(-, Y), G(-)\right)\right](X) & \simeq 0, \\
D_{1}\left[\mu\left(c r_{2} F(-, Y), c r_{2} G(-, Y)\right)\right](X) & \simeq 0 .
\end{aligned}
$$

For the same reason, $D_{1}[\mu(F, G)](X)$ also vanishes. Thus, we see that

$$
\begin{aligned}
& \nabla \mu(F, G)(X, Y)=D_{1}\left[c r_{2} \mu(F, G)(-, Y)\right](X) \oplus D_{1}[\mu(F, G)](X) \\
& \quad \simeq\left(\begin{array}{ccc}
D_{1}[\mu(F(-), G(Y))](X) & \oplus & D_{1}[\mu(F(Y), G(-))](X) \\
\oplus & \oplus \\
D_{1}\left[\mu\left(F(Y), c r_{2} G(-, Y)\right)\right](X) & \oplus & D_{1}\left[\mu\left(c r_{2} F(-, Y), G(Y)\right)\right](X)
\end{array}\right),
\end{aligned}
$$

which, by the bilinearity of $\mu$, is easily seen to be equivalent to $\mu(\nabla F, G) \oplus \mu(F, \nabla G)$.

Proposition 5.6 (Chain rule). Let $\mathcal{A}^{\prime}$ be an abelian category. Let $G: \mathcal{C} \longrightarrow \mathcal{A}^{\prime}$ and $F: \mathcal{A}^{\prime} \longrightarrow C h \mathcal{A}$ be functors, where $G$ is reduced. There is a natural isomorphism of bifunctors that for objects $X, Y \in \mathcal{C}$ yields

$$
\nabla(F \circ G)(X, Y) \cong \nabla F(\nabla G(X, Y), G(Y)) .
$$

To prove the proposition, we use the following lemma.

Lemma 5.7. For functors $F$ and $G$ as in Proposition 5.6,

$$
D_{1}(F \circ G) \simeq D_{1} F \circ D_{1} G .
$$

Proof. Note that $D_{1} G$ is a functor that takes values in $C h_{\geq 0} \mathcal{A}^{\prime}$, whereas the domain of $D_{1} F$ is $\mathcal{A}^{\prime}$. In order to define $D_{1} F \circ D_{1} G$ in a reasonable manner (i.e., so that chain homotopy equivalences are preserved), we use the prolongation of $D_{1} F$. That is, $D_{1} F\left(D_{1} G\right)$ is the functor obtained by applying $D_{1} F$ degreewise to the simplicial replacement of the chain complex used to define $D_{1} G$. (See sections 1 and 2 of JJ-M1 for more details.) However, $D_{1} F$ is additive, and so by lemma 5.4 of [J-M1] $D_{1} F\left(D_{1} G\right)$ is equivalent to the functor obtained by applying $D_{1} F$ degreewise to the chain complex $D_{1} G$. Thus, $D_{1} F \circ D_{1} G$ is equivalent to the total complex of the bicomplex $B(F, G)$ that in bidegree $(p, q)$ is given by $B_{p, q}=\left(\perp_{2}^{p} F\right) \circ\left(\perp_{2}^{q} G\right)$. 
In particular, $B_{0,0}=F \circ G$. Now consider the tricomplex created by applying $C_{*}^{\perp_{2}}$ (as defined in 2.4) to the bicomplex $B(F, G)$, together with the following natural maps of complexes:

$D_{1} F \circ D_{1} G \simeq B(F, G) \stackrel{\alpha}{\longrightarrow} C_{*}^{\perp_{2}} B(F, G) \stackrel{\beta}{\longleftarrow} C_{*}^{\perp_{2}} B_{0,0}(F, G) \simeq D_{1}(F \circ G)$.

We claim that $\alpha$ and $\beta$ are quasi-isomorphisms. To see that $\alpha$ is a quasi-isomorphism, note that since $D_{1} F \circ D_{1} G$ is additive, $B(F, G)$ is as well. It follows that $\perp_{2}^{(k)} B(F, G) \cong 0$ for $k \geq 1$, and so $C_{*}^{\perp_{2}} B(F, G) \simeq B(F, G)$. To see that $\beta$ is a quasi-isomorphism, note that $C_{*}^{\perp_{2}}\left(B_{p, q}(F, G)\right)=D_{1}\left(\left(\perp_{2}^{(p)} F\right) \circ\left(\perp_{2}^{(q)} G\right)\right)$ is acyclic by Lemma 3.3 when $p$ or $q$ is not zero.

Proof of Proposition 5.6. We prove this by computing

$$
\nabla(F \circ G)(X, Y) \text { and } \quad \nabla F(\nabla G(X, Y), G(Y))
$$

directly and comparing the results. We first determine $\nabla F(\nabla G(X, Y), G(Y))$. By Remark 5.2, we have

$$
\nabla F(\nabla G(X, Y), G(Y)) \cong \nabla F\left(D_{1}\left[c r_{2} G(-, Y)\right](X) \oplus D_{1} G(X), G(Y)\right) .
$$

Since $\nabla F$ is additive in its first variable, this is equivalent to

$$
\nabla F\left(D_{1}\left[c r_{2} G(-, Y)\right](X), G(Y)\right) \oplus \nabla F\left(D_{1} G(X), G(Y)\right) .
$$

And, again using 5.2, we see that this is isomorphic to

$$
\begin{array}{ccc}
D_{1}\left[c r_{2} F(-, G(Y))\right]\left(D_{1}\left[c r_{2} G(-, Y)\right]\right)(X) & \oplus & D_{1}\left[c r_{2} F(-, G(Y))\right]\left(D_{1} G(X)\right) \\
\oplus & & \oplus \\
D_{1} F\left(D_{1} c r_{2} G(-, Y)(X)\right) & \oplus & D_{1} F\left(D_{1} G(X)\right) .
\end{array}
$$

By Lemma 5.7 , this is equivalent to

$$
\begin{array}{ccc}
D_{1}\left[\operatorname{cr}_{2} F\left(c r_{2} G(-, Y), G(Y)\right)\right](X) & \oplus & D_{1}\left[c r_{2} F(G(-), G(Y))\right](X) \\
D_{1}\left[F\left(c r_{2} G(-, Y)\right)\right](X) & \oplus & D_{1}[F \circ G](X) .
\end{array}
$$

To compute

$$
\nabla(F \circ G)(X, Y) \cong D_{1}\left[c r_{2}(F \circ G)(-, Y)\right](X) \oplus D_{1}[F \circ G](X),
$$

we first observe that $\mathrm{cr}_{2}(F \circ G)(X, Y)$ is isomorphic to

$$
\begin{array}{ccc}
c r_{2} F\left(c r_{2} G(X, Y), G(Y)\right) & \oplus \quad c r_{2} F(G(X), G(Y)) \\
& & \\
F\left(c r_{2} G(X, Y)\right) & & \\
\oplus & c r_{2} F\left(G(X), c r_{2} G(X, Y)\right) & \oplus r_{3} F\left(G(X), G(Y), c r_{2} G(X, Y)\right) .
\end{array}
$$

Since $D_{1}$ preserves direct sums, it suffices to note that, by Lemma 3.3,

$$
D_{1}\left[c r_{2} F\left(G(-), c r_{2} G(-, Y)\right)\right](X) \simeq 0
$$

and

$$
D_{1}\left[c r_{3} F\left(G(-), G(Y), c r_{2} G(-, Y)\right)\right](X) \simeq 0 .
$$

Hence $\nabla(F \circ G)(X, Y) \cong \nabla F(\nabla G(X, Y), G(Y))$.

We next examine how $\nabla$ behaves with respect to the functors $P_{n}, D_{n}$, and $P_{\infty}$ of our Taylor tower construction. The bifunctor $\nabla F$ is clearly related to $D_{1} F$ in that $D_{1} F(Y)=\nabla_{\star} F(Y)$. By iterating $\nabla$ one can also write $D_{n} F$ in terms of $\nabla F$. 
We will establish this result in Corollary 5.11. To do so we first consider $\nabla^{n} F$ and how to express it via cross effects and $D_{1}$, as we did with $\nabla F$ in remark 5.2.

First, we should make clear what we mean by $\nabla^{n} F$. We can apply $\nabla$ to the bifunctor $\nabla F$ in either of its two variables. However, applying $\nabla$ to the first variable in $\nabla F$ produces the zero functor. Hence, by $\nabla^{2} F$ we will mean the trifunctor obtained by applying $\nabla$ to the second variable in $\nabla F$. The trifunctor $\nabla^{2} F$ is then linear in its first two variables, but not necessarily in its third. In general, we have the following.

Definition 5.8. For a functor $F: \mathcal{C} \rightarrow C h \mathcal{A}$ and objects $X_{1}, X_{2}, \ldots, X_{n}, Y$ in $\mathcal{C}$,

$$
\begin{aligned}
\nabla^{n} F\left(X_{1}, X_{2}, \ldots, X_{n} ; Y\right) & =\nabla_{X_{n}}\left(\nabla^{n-1} F\left(X_{1}, \ldots, X_{n-1} ;-\right)\right)(Y) \\
& =\left(\nabla_{X_{n}} \ldots\left(\nabla_{X_{2}}\left(\nabla_{X_{1}} F\right)\right) \ldots\right)(Y) .
\end{aligned}
$$

That is, $\nabla^{n} F$ is a functor of $n+1$ variables that is degree one in its first $n$ variables and is obtained from $\nabla^{n-1} F$ by applying $\nabla$ to the nonlinear $n$th variable of $\nabla^{n-1} F$.

Using Remark 5.2, we can express $\nabla^{n} F$ in the following way.

Proposition 5.9. For a functor $F: \mathcal{C} \rightarrow C h \mathcal{A}$, there is a natural isomorphism of $(n+1)$-multifunctors

$\nabla^{n} F\left(X_{1}, \ldots, X_{n} ; Y\right) \cong D_{1}^{(n)}\left[c r_{n+1} F(-, \ldots,-, Y) \oplus c r_{n} F(-, \ldots,-)\right]\left(X_{1}, \ldots, X_{n}\right)$, where the symbol $D_{1}^{(n)}$ indicates that we are applying $D_{1}$ to each variable of the $n$-multifunctor separately, as in Definition 3.1.4.

Proof. We begin by noting that, as defined in $3.1 .4, D_{1}^{(n)}=D_{1}^{1} D_{1}^{2} \ldots D_{1}^{n}$, where $D_{1}^{i}$ indicates that one is to apply $D_{1}$ to the functor of one variable obtained by holding all but the $i$ th variable of a multi-functor constant. However, one can show that the order of the $D_{1}^{i}$ 's does not matter. That is, for any $\sigma \in \Sigma_{n}$,

$$
D_{1}^{\sigma(1)} D_{1}^{\sigma(2)} \ldots D_{1}^{\sigma(n)} \cong D_{1}^{n} .
$$

With this in mind, we prove the result by induction. When $n=1$, the result follows by Remark 5.2. Consider $\nabla^{n+1} F\left(X_{1}, \ldots, X_{n+1} ; Y\right)$. By assumption, we have

$$
\nabla^{n+1} F\left(X_{1}, \ldots, X_{n}, X_{n+1} ; Y\right) \cong \nabla_{X_{n+1}}\left(\nabla^{n} F\left(X_{1}, \ldots, X_{n} ;-\right)\right)(Y),
$$

which is isomorphic to

$$
\nabla_{X_{n+1}}\left(D_{1}^{(n)} c r_{n+1} F\left(X_{1}, \ldots, X_{n},-\right)\right)(Y) \oplus \nabla_{X_{n+1}}\left(D_{1}^{(n)} c r_{n} F\left(X_{1}, \ldots, X_{n}\right)\right)(Y) .
$$

However, $D_{1}^{(n)} F\left(X_{1}, \ldots, X_{n}\right)$ is constant with respect to the $(n+1)$ st variable, and so the second summand above is 0 . By Remark 5.2,

$$
\nabla_{X_{n+1}}\left(D_{1}^{(n)} c r_{n+1} F\left(X_{1}, \ldots, X_{n},-\right)\right)(Y)
$$

is isomorphic to

$$
\begin{aligned}
D_{1}^{1} c r_{2}\left[D_{1}^{(n)} c r_{n+1} F\left(X_{1}, \ldots, X_{n},-\right)\right](-, Y)\left(X_{n+1}\right) \\
\oplus D_{1}^{n+1} D_{1}^{(n)} c r_{n+1} F\left(X_{1}, \ldots, X_{n+1}\right) .
\end{aligned}
$$

But it follows from the definition of cross effects that

$$
c r_{2}\left[c r_{n+1} F\left(X_{1}, \ldots, X_{n},-\right)\right]\left(X_{n+1}, Y\right) \cong c r_{n+2} F\left(X_{1}, \ldots, X_{n}, X_{n+1}, Y\right) .
$$

The result then follows from $(*)$ and the fact that the $D_{1}^{i}$ 's commute with one another and with cross effects. 
As a consequence of Proposition 5.9, we have

Corollary 5.10. For $\sigma \in \Sigma_{n}$ and $F: \mathcal{C} \rightarrow C h \mathcal{A}$,

$$
\nabla^{n} F\left(X_{1}, \ldots, X_{n} ; Y\right) \cong \nabla^{n} F\left(X_{\sigma(1)}, \ldots, X_{\sigma(n)} ; Y\right) \text {. }
$$

Moreover, setting $Y=0$ in Proposition 5.9, we obtain

$$
\nabla^{n} F\left(X_{1}, \ldots, X_{n} ; 0\right) \cong D_{1}^{(n)} c r_{n} F\left(X_{1}, \ldots, X_{n}\right) .
$$

Hence by Proposition 3.9, we have the following.

Corollary 5.11. For any functor $F: \mathcal{C} \rightarrow C h \mathcal{A}$,

$$
D_{n} F \simeq \nabla^{n} F(-, \ldots,-; 0)_{h \Sigma_{n}} .
$$

Given this connection, we want to determine how $\nabla$ behaves when applied to $D_{n} F$. We do so by using the equivalence $D_{n} F \simeq\left(D_{1}^{(n)} c r_{n} F\right)_{h \Sigma_{n}}$ of Proposition 3.9. Recall that $D_{1}^{(n)} c r_{n} F$ is a symmetric $n$-multiadditive functor. To describe the effect of $\nabla$ on $D_{n} F$, we define a new functor.

Definition 5.12. Let $F: \mathcal{C} \rightarrow C h \mathcal{A}$. For $X_{1}, \ldots, X_{t} \in \mathcal{C}, 1 \leq t \leq n$, we let $\left.D_{1}^{(n)} c r_{n} F\right|_{\left\{X_{1}, \ldots, X_{t}\right\}}: \mathcal{C} \rightarrow C h \mathcal{A}$ be the functor defined by

$$
\left.D_{1}^{(n)} c r_{n} F\right|_{\left\{X_{1}, \ldots, X_{t}\right\}}(Y)=D_{1}^{(n)} c r_{n} F(X_{1}, X_{2}, \ldots, X_{t}, \overbrace{Y, Y, \cdots, Y}^{n-t \text { times }}) .
$$

Note that $\left.D_{1}^{(n)} c r_{n} F\right|_{\left\{X_{1}, \ldots, X_{t}\right\}}$ is the diagonal of a symmetric $(n-t)$-multiadditive functor.

Proposition 5.13. For any functor $F: \mathcal{C} \rightarrow C h \mathcal{A}$,

$$
\nabla^{t} D_{n} F\left(X_{1}, \ldots, X_{t}\right) \simeq\left(\left.D_{1}^{(n)} c r_{n} F\right|_{\left\{X_{1}, \ldots, X_{t}\right\}}\right)_{h \Sigma_{n-t}} .
$$

Proof. We will prove the proposition in the case where $t=1$. The general case follows by the same argument and induction. Let $G$ denote the symmetric $n$ multiadditive functor $D_{1}^{(n)} c r_{n} F$. From the proof of Lemma 3.4, we see that

$$
c r_{2} G^{\Delta}\left(X_{1}, X_{2}\right) \oplus G^{\Delta}\left(X_{1}\right) \simeq \bigoplus_{\left\{\alpha \in \operatorname{Hom}(\{n\},\{2\}) \mid \alpha^{-1}(1) \neq \emptyset\right\}} G\left(X_{\alpha(1)}, \ldots, X_{\alpha(n)}\right) .
$$

Taking $D_{1}$ with respect to the $X_{1}$ variable, we see by Lemma 3.3 that the only summands that remain are those terms evaluated at exactly one copy of $X_{1}$ and $n-1$ copies of $X_{2}$. In essence, the quadratic and higher order terms vanish under linearization. Thus,

$$
\begin{aligned}
& D_{1}\left(c r_{2} G^{\Delta}\left(-, X_{2}\right) \oplus G^{\Delta}(-)\right)\left(X_{1}\right) \\
& \simeq_{\Sigma_{n}} \bigoplus_{\left\{\alpha \in \operatorname{Hom}(\{n\},\{2\})|| \alpha^{-1}(1) \mid=1\right\}} G\left(X_{\alpha(1)}, \ldots, X_{\alpha(n)}\right) .
\end{aligned}
$$

Let $\Sigma_{n-1}$ be identified with the subgroup of $\Sigma_{n}$ determined by elements that stabilize 1 , that is, $\tau \in \Sigma_{n-1}$ if and only if $\tau(1)=1$. There is a $\Sigma_{n}$-equivariant isomorphism

$$
\begin{aligned}
& Z\left[\Sigma_{n}\right] \otimes_{Z\left[\Sigma_{n-1}\right]} G\left(X_{1}, X_{2}, \cdots, X_{2}\right) \\
& \cong \bigoplus_{\left\{\alpha \in \operatorname{Hom}(\{n\},\{2\})|| \alpha^{-1}(1) \mid=1\right\}} G\left(X_{\alpha(1)}, \ldots, X_{\alpha(n)}\right)
\end{aligned}
$$


given by

$$
\sigma \times t \mapsto(\sigma(1), \sigma * t)
$$

where $\sigma(1)$ represents the unique morphism $\alpha$ that takes $\sigma(1)$ to 1 and all other elements of $\{n\}$ to 2 . Since $D_{1}$ commutes with homotopy orbits, we obtain

$$
\begin{aligned}
\nabla D_{n} F(X, Y) & \simeq \nabla\left(D_{1}^{(n)} c r_{n} F\right)_{h \Sigma_{n}}(X, Y) \\
& =D_{1}\left[c r_{2}\left(D_{1}^{(n)} c r_{n} F\right)_{h \Sigma_{n}}(-, Y) \oplus\left(D_{1}^{(n)} c r_{n} F\right)_{h \Sigma_{n}}(-)\right](X) \\
& \simeq\left(Z\left[\Sigma_{n}\right] \otimes_{Z\left[\Sigma_{n-1}\right]} D_{1}^{(n)} c r_{n} F(X, Y, \cdots, Y)\right)_{h \Sigma_{n}}
\end{aligned}
$$

Then

$$
\left(Z\left[\Sigma_{n}\right] \otimes_{Z\left[\Sigma_{n-1}\right]} D_{1}^{(n)} c r_{n} F(X, Y, \cdots, Y)\right)_{h \Sigma_{n}} \simeq\left(D_{1}^{(n)} c r_{n} F(X, Y \cdots, Y)\right)_{h \Sigma_{n-1}}
$$

by the following version of Shapiro's lemma. If $H$ is a subgroup of $K$, then $E K$ is not only a free $K$-resolution of $Z$ but a free $H$-resolution of $Z$ as well. Hence, for any $H$-module $X$ we have

$$
\begin{aligned}
\left(Z[K] \otimes_{Z[H]} X\right)_{h K} & \simeq Z[E K] \otimes_{Z[K]}\left(Z[K] \otimes_{Z[H]} X\right) \\
& \simeq Z[E K] \otimes_{Z[H]} X \\
& \simeq X_{h H}
\end{aligned}
$$

Our final goals in this section are to prove that $\nabla P_{n} \simeq P_{n-1} \nabla$ and, as a consequence, $\nabla P_{\infty} \simeq P_{\infty} \nabla$. Proving the first equivalence amounts to showing that the difference between $F$ and $P_{n} F$ consists of "terms of order $n+1$ and higher" and that $P_{n-1} \nabla$ takes such terms to 0 . We begin by stating more precisely what we mean by "terms of order $n+1$."

Definition 5.14. Let $k \geq-1$. A functor $F: \mathcal{C} \rightarrow C h \mathcal{A}$ has order $k$, or $F$ is $O(k)$, if $P_{k-1} F \simeq 0$.

Example 5.15. By Lemma 3.3, if $G: \mathcal{C}^{\times k} \rightarrow C h \mathcal{A}$ is a $k$-multireduced functor and $F=G \circ \Delta$, then $F$ is $O(k)$.

Lemma 5.16. The following are equivalent for any functor $F: \mathcal{C} \rightarrow C h \mathcal{A}$ :

a) $F$ is $O(k)$,

b) $P_{k-1} F \simeq 0$

c) $F(*) \simeq 0$ and $D_{t} F \simeq 0$ for all $1 \leq t \leq k-1$,

d) $D_{k} F \stackrel{\simeq}{\longrightarrow} P_{k} F$.

Proof. Clearly, a) and b) are equivalent. The equivalence of b) and d) follows from the exact sequence $D_{k} F \rightarrow P_{k} F \rightarrow P_{k-1} F$. To see that b) implies c), first note that if $P_{k-1} F \simeq 0$, then $P_{t} F \simeq 0$ for all $1 \leq t \leq k-1$ by Remark 2.14.6. Then $F(*) \simeq 0$, since $P_{1} F \simeq 0$ and $P_{1} F \simeq P_{1} c r_{1} F \oplus F(*)$. That $D_{t} F \simeq 0$ for all $1 \leq t \leq k-1$ follows by definition. The exact sequence $D_{t} F \rightarrow P_{t} F \rightarrow P_{t-1} F$ and induction show that c) implies b).

To show that $\nabla P_{n} \simeq P_{n-1} \nabla$, we first show that $\nabla$ reduces the order of certain finite order functors.

Lemma 5.17. Let $G: \mathcal{C}^{\times k} \rightarrow C h \mathcal{A}$ be a $k$-multireduced functor and let $F=G \circ \Delta$. Then $\nabla F$ is $O(k-1)$ in its second variable. 
Proof. The case $k=1$ is trivial, so we may assume $k \geq 2$. By Lemma $3.3, D_{1} F \simeq 0$, and so it suffices to show that the functor (of $Y) D_{1}^{1}\left(c r_{2} F(-, Y)\right)(X)$ is $O(k)$. However,

$$
\begin{gathered}
c r_{2} F\left(X_{1}, X_{2}\right) \cong \bigoplus_{\alpha \in \operatorname{Surj}(\{k\},\{2\})} G\left(X_{\alpha(1)}, \ldots, G_{\alpha(k)}\right) \\
\oplus \bigoplus_{j=1}^{k} \bigoplus_{\beta \in \operatorname{Hom}(\{k-1\},\{2\})} c r_{2}\left[G\left(X_{\beta(1)}, \ldots, X_{\beta(j-1)},-, X_{\beta(j)}, \ldots, X_{\beta(n-1)}\right)\right]\left(X_{1}, X_{2}\right) \\
\oplus \text { higher order terms, }
\end{gathered}
$$

where the higher order terms consist of multiple second order cross effects of $G$. Now consider $D_{1}\left[c r_{2} F\left(-, X_{2}\right)\right]$. Since $D_{1}$ preserves direct sums, it is enough to consider applying $D_{1}$ to each summand in the above decomposition. By Lemma 3.3 , any term containing two or more copies of $X_{1}$ is quasi-isomorphic to 0 after applying $D_{1}$. Hence, the only terms remaining have exactly one copy of $X_{1}$. But these terms must all be evaluated at at least $k-1$ copies of $X_{2}$, and hence (as functors in $X_{2}$ ) have order at least $k-1$ by Example 5.15. Since $P_{k-1}$ preserves direct sums, the direct sum of functors of order $k-1$ is again order $k-1$, and we are done.

This allows us to prove the following.

Proposition 5.18. For all $n \geq 1$,

$$
\nabla P_{n} \simeq P_{n-1} \nabla \text {. }
$$

Proof. For any functor $F: \mathcal{C} \rightarrow C h \mathcal{A}, \nabla P_{n} F$ is degree $n-1$ in its second variable by Proposition 5.4. Hence we have the following natural diagram, where the outer square strictly commutes and the two triangles commute up to homotopy:

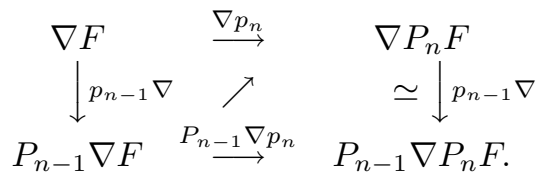

The diagonal arrow in the diagram exists (up to homotopy) by Lemma 2.11. In particular, $\nabla P_{n} F$ is a degree $n-1$ functor with a natural transformation from $\nabla F$, which by Lemma 2.11 must factor through $P_{n-1} \nabla F$. Thus, it suffices to show that $P_{n-1} \nabla p_{n}$ is an equivalence. Since $P_{n}$ and $\nabla$ are linear as functors of functors, it suffices to show that $P_{n-1} \nabla$ of the homotopy fiber of $p_{n}$ is contractible. But, the homotopy fiber of $p_{n}$ is given by the simplicial functor $\perp_{n+1}^{*+1} F$, as indicated in the proof of Proposition 4.8. Since each $\perp_{n+1}^{k+1} F$ is the diagonal of a multireduced functor of at least $n+1$ variables, Lemma 5.17 guarantees that $\nabla \perp_{n+1}^{*+1} F$ is $O(n)$. It follows that $P_{n-1} \nabla \perp_{n+1}^{*+1} F$ is contractible, and so we have the desired equivalence.

We conclude this section with two corollaries of Proposition 5.18.

Corollary 5.19. If $F: \mathcal{C} \rightarrow C h \mathcal{A}$ has order $k$, then $\nabla F$ has order $k-1$.

Corollary 5.20. For any functor $F: \mathcal{C} \rightarrow C h \mathcal{A}$ we have

$$
P_{\infty} \nabla F \simeq \nabla P_{\infty} F
$$


and a diagram that commutes up to homotopy

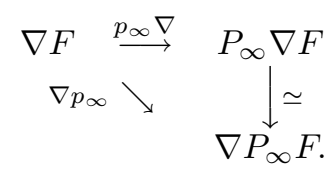

\section{ExAmple: Exponential functors}

Traditionally, after introducing the Taylor series for a general function, one studies the Taylor series of the exponential function, as its derivatives for all $n$ are easily calculated. We will now examine a parallel scenario for similar reasons. We will be working with a commutative ring $\mathbb{K}$ and considering the categories $\mathcal{K}$ of $\mathbb{K}$-modules and $\mathrm{Comm}_{\mathbb{K}}$ of non-unital commutative differential graded $\mathbb{K}$-algebras. We begin by determining the functors from $\mathrm{Comm}_{\mathbb{K}}$ to $C h(\mathcal{K})$ that play the role of exponentials.

Since we tend to normalize our functors to make them reduced, we are really interested in functors whose functional analogues have the form $f(x)=\exp (a x)-1$. For such $f$,

$$
c r_{2} f(x, y)=f(x+y)-f(x)-f(y)=f(x) \cdot f(y) .
$$

Motivated by this, we make the following definition.

Definition 6.2. An exponential functor $F$ from $\mathcal{C}$ to $C h(\mathcal{K})$ is a reduced functor with a natural isomorphism

$$
\alpha_{X, Y}: F(X) \otimes F(Y) \stackrel{\cong}{\longrightarrow} c r_{2} F(X, Y)
$$

such that for all $X, Y \in \mathcal{C}$ the following natural diagram commutes:

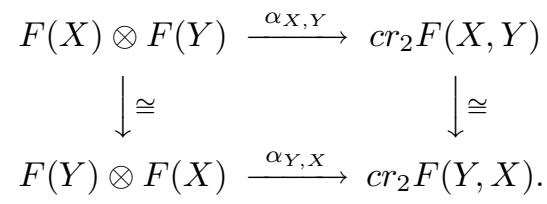

Example 6.3. For $\mathcal{C}$ a small category, Yoneda's lemma implies that the category Func $(\mathcal{C}, \mathcal{K})$ has a set of projective generators given by $\mathbb{K}\left[\operatorname{Hom}_{\mathcal{C}}(X),\right]$ for $X \in \mathcal{C}$. We let $F^{X}$ be the associated reduced functor

$$
F^{X}=\tilde{\mathbb{K}}\left[\operatorname{Hom}_{\mathcal{C}}(X,)\right] .
$$

When $\mathcal{C}$ is an additive category, $F^{X}$ is exponential for all $X \in \mathcal{C}$.

Example 6.4. Let Comm $_{\mathbb{K}}$ be the category of non-unital commutative differential graded $\mathbb{K}$-algebras $\left(\mathrm{Comm}_{\mathbb{K}}\right.$ is naturally isomorphic to the category of augmented unital commutative differential graded $\mathbb{K}$-algebras). Let $\operatorname{Exp}$ be the forgetful functor from $\mathrm{Comm}_{\mathbb{K}}$ to $C h(\mathcal{K})$. Since $X \vee Y=X \oplus Y \oplus X \otimes Y$ for $X, Y \in \mathrm{Comm}_{\mathbb{K}}$, Exp is exponential.

We recall that any analytic function $f$ that satisfies (6.1) must be of the form

$$
\exp (a x)-1=[\exp (-)-1] \circ(a \cdot x)
$$

for some $a$. Since $a \cdot x$ is a linear function in that it preserves addition and is reduced, we see that any function $f$ satisfying (6.1) is the composite of a linear function with a universal function. A similar fact is true for functors. 
Lemma 6.5. Every exponential functor $F: \mathcal{C} \longrightarrow C h(\mathcal{K})$ factors as $F=\operatorname{Exp} \circ A$ for $A$ a reduced coproduct-preserving functor from $\mathcal{C}$ to Comm $_{\mathbb{K}}$.

Proof. We define a product $F(X) \otimes F(X) \stackrel{\mu}{\longrightarrow} F(X)$ by the composite

$$
F(X) \otimes F(X) \stackrel{\alpha_{X}}{\longrightarrow} c r_{2} F(X) \stackrel{i}{\longrightarrow} F(X \vee X) \stackrel{F(+)}{\longrightarrow} F(X),
$$

where $c r_{2} F(X)$ denotes $c r_{2} F(X, X)$. Since $F$ is exponential, this product is well defined and commutative. We are left to establish that $\mu$ is associative, since it then easily follows that $A(X)=\{F(X), \mu\}$ is a coproduct-preserving functor. Consider the diagram

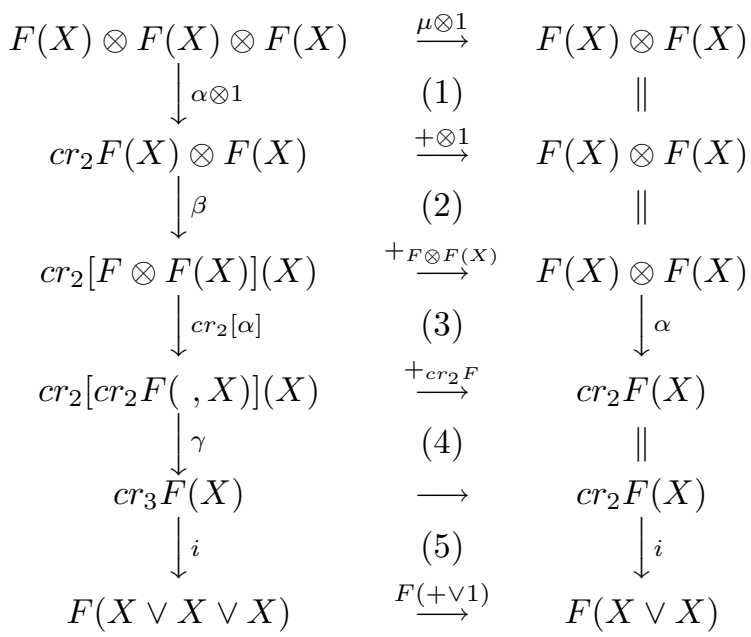

where all the vertical maps except those marked $i$ are isomorphisms. The first, third and fifth squares commute by naturality. The fourth square, with the map $\gamma$, commutes by definition. The isomorphism $\beta$ is the general isomorphism given by the composite

$$
c r_{2} F(X) \otimes M \stackrel{i \otimes 1}{\longrightarrow} F(X \vee X) \otimes M \stackrel{\pi}{\longrightarrow} c r_{2}(F \otimes M)(X)
$$

applied to $M=F(X)$. Square (2) does not commute until one applies $F(+)$. To prove this, one considers the diagram

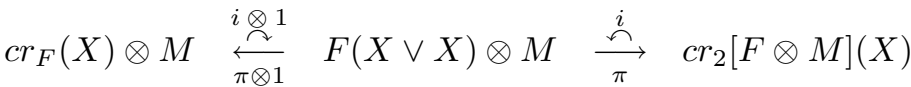

$$
\begin{aligned}
& \downarrow+ \\
& F(X) \otimes M
\end{aligned}
$$

and shows that $+\circ(i \otimes 1)=+\circ i \circ \pi \circ(i \otimes 1)$. This is true because the images of $i \otimes 1$ and $i$ are both equal to the elements fixed by the idempotent $i d-F\left(i_{1} \pi_{1}+i_{2} \pi_{2}\right) \otimes M$. From this diagram and the related diagram beginning with $(1 \otimes \alpha)$, one determines that $\mu$ is an associative product.

We next observe that to understand the Taylor series for an exponential functor $F$ we only need to understand the Taylor series for $\operatorname{Exp}$ and identify the functor $A$. This is because of the following lemma, whose proof follows from the definition of $\perp_{n+1}$. 
Lemma 6.6. If $A: \mathcal{C}^{\prime} \longrightarrow \mathcal{C}$ is a reduced coproduct-preserving functor and $F$ : $\mathcal{C} \longrightarrow C h(\mathcal{K})$ is a functor, then

$$
P_{n}(F \circ A) \cong\left(P_{n} F\right) \circ A .
$$

We begin our investigation of $P_{n} \operatorname{Exp}$ by observing that

$$
\begin{aligned}
H_{0}\left(P_{n} \mathbf{E x p}\right)(X) & =p_{n} H_{0} \mathbf{E x p}(X) \\
& =\operatorname{cokernel}\left(c r_{n+1} H_{0} \mathbf{E x p}(X) \stackrel{+}{\longrightarrow} H_{0} \operatorname{Exp}(X)\right) \\
& =\operatorname{cokernel}\left(H_{0} X^{\otimes(n+1)} \stackrel{\mu}{\longrightarrow} H_{0} X\right) \\
& =H_{0}(X) / H_{0}(X)^{(n+1)} .
\end{aligned}
$$

The structure maps $p_{n+1} \operatorname{Exp} \longrightarrow p_{n} \operatorname{Exp}$ are the usual restriction maps. It follows that $H_{0} P_{\infty} \mathbf{E x p}=H_{0}(X)^{\wedge}$ (the completion of the algebra $H_{0}(X)$ ).

We compute the differentials for $\operatorname{Exp}$ to be

$$
\begin{aligned}
\nabla \operatorname{Exp}(X, Y) & =D_{1}\left[c r_{2} \operatorname{Exp}(\star, Y) \oplus \mathbf{E x p}(\star)\right](X) \\
& \cong D_{1}[\mathbf{E x p}(\star) \otimes \mathbf{E x p}(Y) \oplus \mathbf{E x p}(\star)](X) \\
& \cong D_{1} \mathbf{E x p}(\star)(X) \otimes[\mathbf{E x p}(Y) \oplus \mathbb{K}] .
\end{aligned}
$$

It follows from Proposition 5.9 and Corollary 5.11 that

$$
\nabla^{n} \operatorname{Exp}\left(X_{1}, \ldots, X_{n}, Y\right) \cong \bigotimes_{i=1}^{n} D_{1} \operatorname{Exp}(\star)\left(X_{i}\right) \otimes[\operatorname{Exp}(Y) \oplus \mathbb{K}]
$$

and

$$
D_{n} \mathbf{E x p} \cong\left(\bigotimes^{n} D_{1} \mathbf{E x p}\right)_{h \Sigma_{n}} .
$$

Remark 6.7. Note that $f(x)=\exp (a x)-1$ is the unique solution to the differential equation

$$
\frac{d}{d x} f=a \cdot(f+1), \quad f(0)=0,
$$

where $\frac{d}{d x} f(y)=\nabla f(1, y)$. Similarly, if $\mathcal{C}$ has a projective generator $\mathbb{C}$ and $F$ is an exponential functor, then it satisfies the differential equation

$$
\frac{d}{d \mathbb{C}} F=\nabla F(\mathbb{C},) \cong D_{1} F(\mathbb{C}) \cdot(F \oplus \mathbb{K}), \quad F(0)=0 .
$$

The functor $G=\prod_{n=1}^{\infty} D_{n} F^{X}$ satisfies the differential equation (6.8), but the functors $F^{X}$ are generally not of this form since their Taylor towers do not split. Using examples like these, one can construct an uncountable family of non-equivalent functors that satisfy the differential equation $(6.8)$ for a fixed $D_{1} F(\mathbb{C})$.

Different towers. In defining the Taylor tower in section 2 , the only requirements we placed on the domain categories of functors were that they be basepointed and contain finite coproducts. Working in such generality allowed us to establish the essential properties of the Taylor tower functors quickly and cleanly without becoming bogged down by concerns about preserving specific properties of the domain category. However, when the domain category has additional structure, this general approach also means that $P_{n} F$ may not behave as desired with respect to the extra structure. For example, when working with functors of modules over a ring $R$, one might hope to produce functors that preserve short exact sequences, 
or take them to long exact sequences in homology. But, for the additive functor $T_{\mathbb{Z} / 2}=-\otimes_{\mathbb{Z}} \mathbb{Z} / 2$ on $A b$, the category of abelian groups, we have $P_{1} T_{\mathbb{Z} / 2} \cong T_{\mathbb{Z} / 2}$, which does not preserve short exact sequences in general. The usual solution to this problem is to replace modules by projective resolutions. That is, we define $P_{1}^{d} T_{\mathbb{Z} / 2}$ by

$$
P_{1}^{d} T_{\mathbb{Z} / 2}(X)=P_{1} T_{\mathbb{Z} / 2}\left(P_{*}\right),
$$

where $P_{*} \rightarrow X$ is a projective resolution. This new functor $P_{1}^{d} T_{\mathbb{Z} / 2}$ takes short exact sequences to long exact sequences in homology, but is not the same as $P_{1} T_{\mathbb{Z} / 2}$ : for an abelian group $A$ we have $H_{*} P_{1} T_{\mathbb{Z} / 2}(A)=0$ for $*>0$, whereas $H_{*} P_{1}^{d} T_{\mathbb{Z} / 2}(A) \cong$ $\operatorname{Tor}_{*}^{\mathbb{Z}}(A, \mathbb{Z} / 2)$.

In working with the closed model category $\mathrm{Comm}_{\mathbb{K}}$ we are faced with a similar problem. In this case, we would like, as one usually does with closed model categories, to work up to weak equivalence. That is, if a map $f: A \rightarrow A^{\prime}$ of DGA's is a weak equivalence (a quasi-isomorphism of DG objects), we want $P_{n} \mathbf{E x p}(f)$ to be a quasi-isomorphism as well. This is not always the case. The reason that $P_{1} \mathbf{E x p}$ does not preserve weak equivalences stems from the fact that coproducts do not preserve weak equivalences in $\mathrm{Comm}_{\mathbb{K}}$. This implies that $\perp_{n+1} \mathbf{E x p}$, as a direct summand of $\operatorname{Exp} \circ\left(\bigvee_{i=1}^{n+1}-\right)$, does not preserve weak equivalences, and, as a consequence, $P_{n} \mathbf{E x p}$ does not preserve weak equivalences. This problem is fixed, as in the previous paragraph, by evaluating $P_{n} \operatorname{Exp}$ on weakly equivalent cofibrant approximations of objects. Since the coproduct in $\mathrm{Comm}_{\mathbb{K}}$ preserves weak equivalences of cofibrant objects, the result will be a functor that preserves weak equivalences. This process can be used in any setting in which a functorial cofibrant replacement functor exists. We will illustrate how to do this in the case of Exp below by constructing another degree $n$ functor $P_{n}^{w} \mathbf{E x p}$ that preserves weak equivalences and fits into a natural commutative diagram

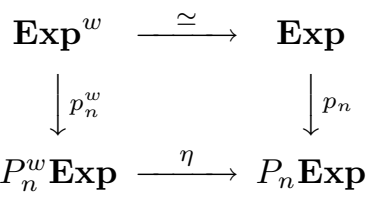

where $P_{n}^{w} \operatorname{Exp} \stackrel{\eta}{\longrightarrow} P_{n} \operatorname{Exp}$ is an equivalence for cofibrant objects of Comm $_{\mathbb{K}}$ but is not an equivalence generally. We then go on to show how $P_{n}^{w} \mathbf{E x p}$ can be considered as the derived functor of $I / I^{n}$ (when suitably interpreted in model category theory terms). We do this in detail when $\mathbb{Q} \subset \mathbb{K}$, and indicate what needs to be done more generally. This program has been carried out by V. Minasian in $[\mathrm{M}]$ using the model category of ring spectra from [BKMM].

A functorial free resolution. We first recall that there is a sequence of adjoint pairs of functors (the top arrow is right adjoint to the bottom arrow)

$$
\operatorname{Comm}_{\mathbb{K}} \underset{U=\mathbf{E}}{\stackrel{S}{\leftrightarrows}} \operatorname{Ch}(\mathcal{K}) \underset{\Gamma}{\stackrel{N}{\leftrightarrows}} \operatorname{Simp}(\mathcal{K}) \underset{U}{\stackrel{\tilde{\mathbb{K}}[]}{\curvearrowleft}} \operatorname{Simp}\left(\operatorname{Sets}_{*}\right)
$$

and that the symmetric functor $S$ is given by

$$
S(X)=T(X) / \sim, \quad T(X)=\bigoplus_{n=1}^{\infty} \bigotimes^{n} X, \quad x \cdot y \sim(-1)^{|x||y|} y \cdot x,
$$


or, equivalently, by

$$
\begin{gathered}
S(X) \cong \bigoplus_{n=1}^{\infty} S^{n}(X), \quad S^{n}(X)=T^{n}(X) / \Sigma_{n}, \\
\sigma *\left(a_{1} \otimes \cdots \otimes a_{n}\right)=(-1)^{?}\left(a_{\sigma(1)} \otimes \cdots \otimes a_{\sigma(n)}\right),
\end{gathered}
$$

where the sign is determined by the relation

$$
(i i+1)\left(a_{1} \otimes \cdots \otimes a_{n}\right)=(-1)^{\left|a_{i}\right|\left|a_{i+1}\right|}\left(a_{1} \otimes \cdots \otimes a_{i+1} \otimes a_{i} \otimes \cdots \otimes a_{n}\right) .
$$

We write $K$ for the composite functor from Comm $_{\mathbb{K}}$ to itself given by

$$
K=S \circ N \circ \tilde{\mathbb{K}}[] \circ U \circ \Gamma \circ U,
$$

and note that $K$ is a cotriple on Comm $\mathbb{K}_{\mathbb{K}}$. There is a natural map as pointed sets from $\operatorname{Id}_{\mathrm{Comm}_{\mathbb{K}}}$ to $K$ which is a section to the counit, and so, for any $A \in \mathrm{Comm}_{\mathbb{K}}$,

$$
\operatorname{Exp}(\epsilon): \operatorname{Exp}\left(K^{*+1}(A)\right) \stackrel{\simeq}{\longrightarrow} \operatorname{Exp}(A) .
$$

Since the construction $P_{n}$ preserves equivalences of functors, we see that

$$
P_{n}\left[\operatorname{Exp}\left(K^{*+1}\right)\right] \stackrel{\simeq}{\longrightarrow} P_{n} \operatorname{Exp} .
$$

As a result, $P_{n}\left[\operatorname{Exp}\left(K^{*+1}\right)\right]$ does not preserve weak equivalences, since $P_{n} \mathbf{E x p}$ does not. Instead, we see below that composing $P_{n} \operatorname{Exp}$ with $K^{*+1}$ produces the desired weak-equivalence-preserving functor.

Remark 6.10. In general, $K^{*+1} A \vee K^{*+1} B \stackrel{千}{\longrightarrow} K^{*+1}(A \vee B) \stackrel{\simeq}{\longrightarrow} A \vee B$. For example, let $A=\mathbb{Z} / p$ with the usual ring multiplication in $\mathrm{Comm}_{\mathbb{Z}}$. Then

$$
H_{t}\left(K^{*+1} \mathbb{Z} / p \vee K^{*+1} B\right)= \begin{cases}\mathbb{Z} / p \vee H_{0}(B) & \text { if } t=0, \\ \operatorname{Tor}_{t}^{\mathbb{Z}}(\mathbb{Z} / p, B) & \text { otherwise. }\end{cases}
$$

More generally,

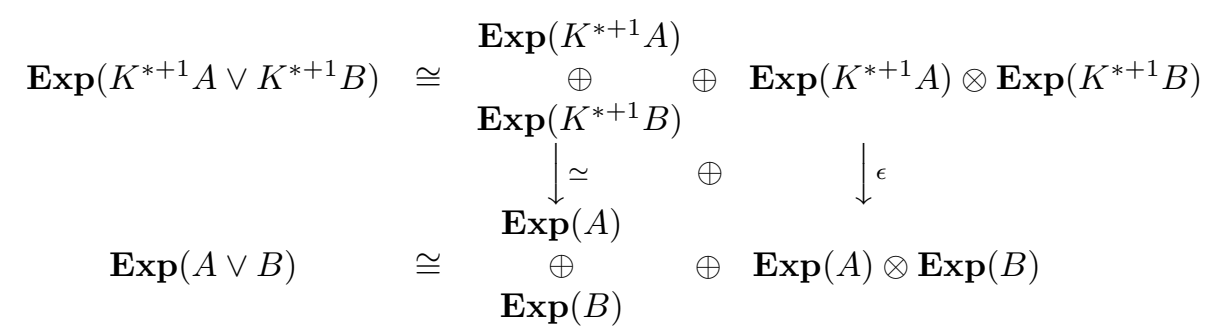

and there is a convergent spectral sequence

$$
E_{p, q}^{2}=\bigoplus_{q^{\prime}+q^{\prime \prime}=q} \operatorname{Tor}_{p}^{q}\left(H_{q^{\prime}}(\operatorname{Exp} A), H_{q^{\prime \prime}}(\operatorname{Exp} B)\right) \Rightarrow \begin{gathered}
\operatorname{Tor}_{p+q}^{\mathbb{K}}(\operatorname{Exp}(A), \mathbf{E x p}(B)) \\
\| \\
H_{p+q}\left(\operatorname{Exp}\left(K^{*+1} A\right) \otimes \mathbf{E x p}\left(K^{*+1} B\right)\right)
\end{gathered} .
$$

In particular, if $f: A \longrightarrow A^{\prime}$ and $g: B \longrightarrow B^{\prime}$ are weak equivalences, then the map $K^{*+1}(f) \vee K^{*+1}(g)$ is a weak equivalence as well. Hence, if $f: A \longrightarrow A^{\prime}$ is a weak equivalence, then $\bigvee_{i=1}^{n+1} K^{*+1}(f): \bigvee_{i=1}^{n+1} K^{*+1}(A) \longrightarrow \bigvee_{i=1}^{n+1} K^{*+1}\left(A^{\prime}\right)$ is a weak equivalence. It follows that $P_{n} \operatorname{Exp}\left(K^{*+1}(f)\right): P_{n} \operatorname{Exp}\left(K^{*+1}(A)\right) \longrightarrow$ $P_{n} \operatorname{Exp}\left(K^{*+1}\left(A^{\prime}\right)\right)$ is a weak equivalence as well. 
Definition 6.11. We define $\operatorname{Exp}^{w}$ to be the functor $\operatorname{Exp}\left(K^{*+1}\right)$ and $P_{n}^{w} \mathbf{E x p}$ to be the functor $\left[P_{n} \operatorname{Exp}\right]\left(K^{*+1}\right)$. We let $p_{n}^{w}$ be the natural transformation $\left[p_{n}\right]\left(K^{*+1}\right)$ : $\mathbf{E x p}^{w} \longrightarrow P_{n}^{w} \operatorname{Exp}$. We note that $P_{n}^{w} \mathbf{E x p}$ is degree $n$, preserves weak equivalences and makes the diagram (6.9) commute. Of course, $\left(P_{n}^{w}(F), p_{n}^{w}\right)$ can be defined for any functor from $\mathrm{Comm}_{\mathbb{K}}$ to $C h(\mathcal{K})$, and satisfies the expected universal properties.

The functors $I / I^{n}$. The $n$-fold tensor product of $K^{*+1}$ is an $n$-multisimplicial object of $\mathrm{Comm}_{\mathbb{K}}$. We use its associated diagonal object. We define $I / I^{n}$ to be the functor from $\mathrm{Comm}_{\mathbb{K}}$ to $C h(\mathcal{K})$ given by sending $A \in \mathrm{Comm}_{\mathbb{K}}$ to

$$
I / I^{n}(A)=\text { cokernel }\left[\operatorname{Exp}\left(K^{*+1} A\right)^{\otimes n} \simeq \operatorname{Exp}\left(K^{*+1}(A) \stackrel{\otimes n}{ }\right) \stackrel{\operatorname{Exp}(\mu)}{\longrightarrow} \operatorname{Exp}\left(K^{*+1} A\right)\right] .
$$

We first note that $\operatorname{Exp}(\mu)$ is levelwise injective, and hence this cokernel is also the homotopy cofiber of the map $\operatorname{Exp}(\mu)$. We also note that $I / I^{n}$ is naturally equivalent to the homotopy fiber of the $n$-multiplication map, and hence is the homological analogue of the usual functor $I / I^{n}$ for algebras.

Proposition 6.12. The functor $I / I^{n}$ is degree $n-1$.

Proof. Since $I / I^{n}$ preserves weak equivalences, it also behaves well with respect to $K$-resolutions, so that it suffices to show $I / I^{n}$ is degree $n-1$ when evaluated on the image of $K$. That is, it suffices to show that

$$
c r_{n}\left(I / I^{n}\right)(K(A), \ldots, K(A)) \simeq *
$$

for all $A \in \operatorname{Comm}_{\mathbb{K}}$. Since $K=(S \circ N \circ \tilde{\mathbb{K}}[]) \circ(U \circ \Gamma \circ U)$ and $(S \circ N \circ \tilde{\mathbb{K}}[])$ preserves coproducts (it has a right adjoint),

$$
\bigvee^{n} K(A) \cong(S \circ N \circ \tilde{\mathbb{K}}[]) \bigvee^{n}[U \circ \Gamma \circ U(A)]
$$

Thus it is enough to show that $I / I^{n} \circ(S \circ N \circ \tilde{\mathbb{K}}[])$ is degree $n-1$ as a functor from pointed simplicial sets to $C h(\mathcal{K})$. Since $(S \circ N \circ \tilde{\mathbb{K}}[X])$ is always $K$-projective, $I / I^{n}(S \circ N \circ \tilde{\mathbb{K}}[])$ is naturally simplicial homotopy equivalent to $J / J^{n}(S \circ N \circ \tilde{\mathbb{K}}[])$, where

It follows that

$$
J / J^{n}(A)=\text { cokernel }\left[\operatorname{Exp}\left(A^{\otimes n}\right) \stackrel{\operatorname{Exp}(\mu)}{\longrightarrow} \operatorname{Exp}(A)\right] .
$$

$$
J / J^{n} \circ(S \circ N \circ \tilde{\mathbb{K}}[])=\bigoplus_{k=1}^{n-1} S^{k}(N \circ \tilde{\mathbb{K}}[]) \simeq \bigoplus_{k=1}^{n-1} \tilde{\mathbb{K}}\left[\bigwedge^{n}() / \Sigma_{n}\right],
$$

where $\Sigma_{n}$ acts on $\Lambda^{n}(X)$ by simply permuting the factors. We compute

$$
\begin{aligned}
& \bigwedge^{n}\left(\bigvee_{k=1}^{n} X_{i}\right) \cong \underset{\gamma \in \operatorname{Map}_{\text {Sets }}(\{n\},\{k\})}{ } X_{\gamma(1)} \wedge \cdots \wedge X_{\gamma(n)} \\
& \Downarrow \\
& c r_{n}\left(\wedge^{n}\right)\left(X_{1}, \ldots, X_{n}\right) \cong \bigvee_{\substack{\gamma \in \operatorname{Surj}_{\text {Sets }}(\{n\},\{k\}) \\
\Downarrow}} X_{\gamma(1)} \wedge \cdots \wedge X_{\gamma(n)} \\
& \operatorname{cr}_{n} \tilde{K}\left[\bigwedge^{n}() / \Sigma_{n}\right]\left(X_{1}, \ldots, X_{n}\right) \cong \tilde{K}\left[X_{1}\right] \otimes \tilde{K}\left[X_{2}\right] \otimes \cdots \otimes \tilde{K}\left[X_{n}\right] .
\end{aligned}
$$

Since $\tilde{K}[]$ is degree $1, c r_{n} \tilde{K}\left[\bigwedge^{n}() / \Sigma_{n}\right]$ is $n$-multiadditive and so $\tilde{K}\left[\bigwedge^{n}() / \Sigma_{n}\right]$ is degree $n$. 
There is a natural transformation $\tau$ from $P_{n}^{w} \operatorname{Exp}$ to $J / J^{n+1}$ (obtained essentially by taking $H_{0}$ along the $P_{n}$ direction) and hence a natural diagram

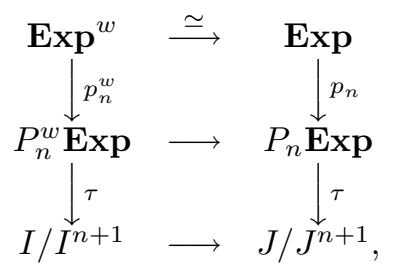

where $I / I^{n+1}$ is a degree $n$ functor.

The next result and its corollary are also established in $[\mathrm{K}-\mathrm{Mc}$.

Proposition 6.13. If $\mathbb{Q} \subseteq \mathbb{K}$, then $\tau: P_{n}^{w} \operatorname{Exp} \stackrel{\simeq}{\longrightarrow} I / I^{n+1}$.

Remark. In fact, $\tau$ is an equivalence if and only if $\mathbb{Q} \subseteq \mathbb{K}$ (see 6.17).

Proof. Since both functors preserve equivalences, it is enough to show the result when evaluated at $K(A)$ for some $A$. Thus, it suffices to show that

$$
P_{n} \operatorname{Exp}(S \circ N \circ \tilde{\mathbb{K}}[]) \stackrel{\simeq}{\longrightarrow} J / J^{n+1}(S \circ N \circ \tilde{\mathbb{K}}[])
$$

as functors from pointed simplicial sets. By induction, we need only show that the induced maps

$$
D_{n} \operatorname{Exp}(S \circ N \circ \tilde{\mathbb{K}}[]) \longrightarrow \operatorname{hofiber}\left\langle J / J^{n+1}(S \circ N \circ \tilde{\mathbb{K}}[]) \longrightarrow J / J^{n}(S \circ N \circ \tilde{\mathbb{K}}[])\right\rangle
$$

are equivalences. These are the maps

$$
\begin{gathered}
\left.\left(\bigotimes_{1}^{n} D_{k=1}^{\infty} \tilde{\mathbb{K}}\left[\bigwedge^{k}() / \Sigma_{k}\right]\right)\right)_{h \Sigma_{n}} \\
\downarrow\left(\otimes^{n} D_{1}(\pi)\right)_{h \Sigma_{n}} \\
\left(\bigotimes^{n} D_{1}\left(\tilde{\mathbb{K}}\left[\bigwedge^{1}() / \Sigma_{1}\right]\right)\right)_{h \Sigma_{n}} \\
\downarrow
\end{gathered}
$$

$(\star)$

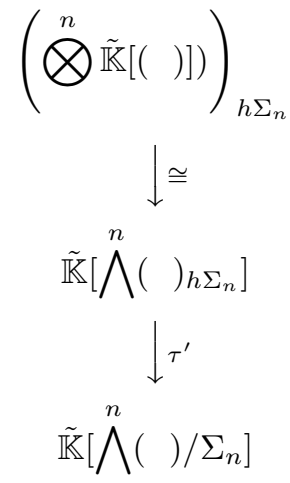

where $\tau^{\prime}$ is the natural map from homotopy orbits to orbits which is an equivalence whenever $\left|\Sigma_{n}\right|=n$ ! is invertible in $\mathbb{K}$. 
Since homotopy orbits preserve equivalences and $D_{1}$ preserves direct sums of functors, it suffices to establish that if $k$ ! is invertible in $\mathbb{K}$ then $D_{1}\left(\tilde{\mathbb{K}}\left[\wedge^{k}() / \Sigma_{k}\right]\right) \simeq$ $*$ for $k \geq 2$. However, since

$$
\left(\bigotimes^{k} \tilde{\mathbb{K}}[]\right)_{h \Sigma_{k}} \cong \tilde{\mathbb{K}}\left[\bigwedge^{k}()_{h \Sigma_{k}}\right] \stackrel{\tau^{\prime}}{\longrightarrow} \tilde{\mathbb{K}}\left[\bigwedge^{k}() / \Sigma_{k}\right]
$$

and $\left(\otimes^{k} \tilde{\mathbb{K}}[]\right)_{h \Sigma_{k}}$ is a homogeneous functor of degree $n$ (see 3.10), the result follows from the fact that $D_{1}$ preserves equivalences.

Corollary 6.14. Let $A$ be $a \mathbb{K}$-algebra and $I_{A}=\operatorname{kernel}[A \otimes A \stackrel{\mu}{\longrightarrow} A] \in \operatorname{Comm}_{A}$. Then

$$
H_{*}\left(P_{1}^{w} \operatorname{Exp}\left(I_{A}\right)\right)=A Q_{*}(A / \mathbb{K}),
$$

where $A Q_{*}$ is the André-Quillen homology of A. The third quadrant spectral sequence for $\operatorname{holim}_{n} P_{n}^{w} \operatorname{Exp}\left(I_{A}\right)$ is Quillen's fundamental spectral sequence for $A$ (Q2).

It is not the case, in general, that $P_{1}^{w}$ is related to André-Quillen or Quillen homology. We offer two examples below, one in which $P_{1}^{w} F$ agrees with Quillen homology and one in which it does not.

Example 6.15. If one considers rings that do not contain $\mathbb{Q}, H_{*}\left(P_{1}^{w} \mathbf{E x p}\left(I_{A}\right)\right)$ may not be the same as André-Quillen homology. For example, for $\mathbb{K}=\mathbb{F}_{p}$,

$$
A Q_{*}\left(\mathbb{F}_{p}[x] / \mathbb{F}_{p}\right) \begin{cases}\mathbb{F}_{p} & \text { if } *=0, \\ 0 & \text { otherwise. }\end{cases}
$$

On the other hand, it was established in $[\mathrm{B}-\mathrm{M}]$ that $P_{1}^{w} \operatorname{Exp}\left(I_{\mathbb{F}_{p}[x]}\right)$ is equivalent to the topological André-Quillen homology $(T A Q)$ of $\mathbb{F}_{p}[x]$. However, $T A Q\left(\mathbb{F}_{p}[x]\right)$ is known to be equivalent to the smash product of the Eilenberg-Mac Lane spectra $H \mathbb{F}_{p}$ and $H \mathbb{Z}([\mathbb{R})$. This smash product has nontrivial homology in dimensions greater than 0 , and as a result, $A Q_{*}\left(\mathbb{F}_{p}[x] / \mathbb{F}_{p}\right) \neq P_{1}^{w} \operatorname{Exp}\left(\mathbb{F}_{p}[x]\right)$.

Example 6.16. There are categories other than $\operatorname{Comm}_{\mathbb{Q} *}$ for which $P_{1}^{w}$ and Quillen homology are related. Let $\mathcal{G}$ be the category of groups and $A b$ the category of abelian groups. The abelianization functor $L: \mathcal{G} \rightarrow A b$ is the left adjoint to the forgetful functor from $A b$ to $\mathcal{G}$. Recall that for a group $A$,

$$
L(A) \cong H_{1}(B . A) \cong H_{0}(A ; \mathbb{Z}) .
$$

Then for groups $A$ and $A^{\prime}$, by Seifert-Van Kampen, we have

$$
\begin{aligned}
L\left(A * A^{\prime}\right) & =H_{1}\left(B \cdot\left(A * A^{\prime}\right) ; \mathbb{Z}\right) \\
& \cong H_{1}\left(B \cdot A \vee B \cdot A^{\prime} ; \mathbb{Z}\right) \\
& \cong H_{1}(B \cdot A ; \mathbb{Z}) \oplus H_{1}\left(B \cdot A^{\prime} ; \mathbb{Z}\right) \\
& \cong L(A) \oplus L\left(A^{\prime}\right) .
\end{aligned}
$$

In other words, $L$ is additive and $P_{1} L \cong L$. Moreover, as a functor to abelian groups, $H_{*} P_{1} L=0$ for $*>0$. For an object $A$ in $\mathcal{A}$, the Quillen homology of $A$ 
is the homology of $L\left(P_{*}\right)$ for a projective resolution $P_{*}$ of $A$. Hence, in this case, Quillen homology is the same as the homology of $P_{1}^{w} L$. Furthermore, the Kan loop group $G$ of $B . A$ is a free resolution of $A$, and so

$$
\begin{aligned}
H_{*} P_{1}^{w} L(A) & =H_{*}[L(G B \cdot A)] \\
& \cong H_{*+1}(B \cdot A ; \mathbb{Z}) \\
& \cong H_{*}(A ; \mathbb{Z}) .
\end{aligned}
$$

As $H_{*}(A ; \mathbb{Z})$ is generally nonzero for $*>0$, we see that $P_{1} L \neq P_{1}^{w} L$.

Remarks about the general case: $E_{\infty}$-algebras. By the $\Sigma_{2}$ equivariant cofibration sequence of pointed simplicial sets,

$$
X \stackrel{\Delta}{\longrightarrow} X \wedge X \stackrel{\pi}{\longrightarrow}\left(\begin{array}{c}
X \\
2
\end{array}\right)
$$

we obtain a diagram of cofibrations

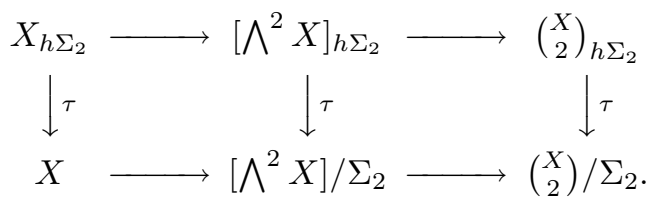

Since $\left(\begin{array}{c}X \\ 2\end{array}\right)$ is $\Sigma_{2}$ free, the vertical map on the right is an equivalence. Taking $D_{1}(\tilde{\mathbb{K}}[])$, we obtain a diagram of fibrations (up to equivalence)

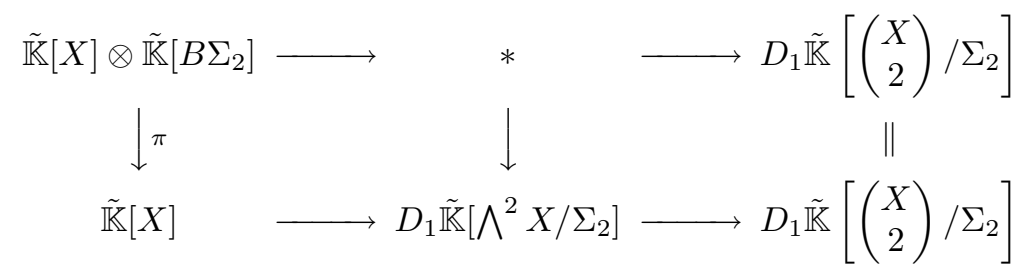

and hence there is a convergent first quadrant spectral sequence

$$
E_{p, q}^{2}=H_{p+1}\left(\Sigma_{2} ; H_{q}(X ; \mathbb{K})\right) \Rightarrow H_{p+q}\left(D_{1} \tilde{\mathbb{K}}\left[\bigwedge^{2} X / \Sigma_{2}\right]\right)
$$

(where the action of $\Sigma_{2}$ is trivial on the homology of $X$ ). In particular,

$$
\tilde{\mathbb{K}}\left[\bigwedge^{2} X / \Sigma_{2}\right] \text { is homogeneous } \Leftrightarrow \frac{1}{2} \in \mathbb{K} .
$$

More generally, one can show that $\tilde{\mathbb{K}}\left[\wedge^{n} X / \Sigma_{n}\right]$ is homogeneous if and only if $\frac{1}{n !} \in$ $\mathbb{K}$.

Since Proposition 6.13 is true if and only if $(\star)$ is true, we see that Proposition 6.13 is true if and only if $\mathbb{Q} \subseteq \mathbb{K}$.

One could "fix" this difficulty if one could replace the functor $S$ used to construct the functor $K$ by one which takes homotopy orbits instead of strict orbits - that is, if one could define $S^{w}: C h(\mathcal{K}) \longrightarrow \mathrm{Comm}_{\mathbb{K}}$, where

$$
S^{w}(X) \simeq \bigoplus_{n=1}^{\infty}\left(T^{n}(X)\right)_{h \Sigma_{n}}
$$


and use $S^{w}$ to construct our functor $K$. Of course, this can't be done for strictly commutative algebras, but V. Minasian has shown how to do it for strongly homotopy commutative, or $E_{\infty}$-algebras, in $[\mathrm{M}]$. We outline the general approach here and refer the reader to $[\mathrm{M}]$ for the complete story. Part I of [K-M] provides a nice introduction to $E_{\infty}$ structures for those who are interested.

One can consider Comm $\mathrm{K}_{\mathbb{K}}$ as a full and faithful subcategory of the category of $E_{\infty} \mathbb{K}$-algebras, $E_{\infty}(\mathbb{K})$ (for a description of the $E_{\infty}$-operad structure, see $[\bar{K}-\mathrm{M}]$ ). We will once again write $\operatorname{Exp}$ for the forgetful functor from $E_{\infty}(\mathbb{K})$ to $C h(\mathcal{K})$. This functor has a left adjoint $S^{w}$ of the form we desire. The explicit construction of $S^{w}$ depends upon the model one chooses to encode the higher homotopy compatibility conditions in $E_{\infty}(\mathbb{K})$. The main point for our purposes is that the composite functor $\operatorname{Exp}\left(S^{w} \circ N \circ \tilde{\mathbb{K}}[]\right)$ is an infinite direct sum of homogeneous functors. Independent of the models chosen, $(\star)$ becomes equivalent to the sequence

$$
\begin{aligned}
& \left(\bigotimes^{n} D_{1}\left(\bigoplus_{k=1}^{\infty} \tilde{\mathbb{K}}\left[\left(\bigwedge^{k}\right)_{h \Sigma_{k}}\right]\right)\right)_{h \Sigma_{n}} \\
& \downarrow\left(\bigotimes^{n} D_{1}(\pi)\right)_{h \Sigma_{n}} \\
& \left(\bigotimes ^ { n } D _ { 1 } \left(\tilde{\mathbb{K}}\left[\left(\bigwedge_{h \Sigma_{1}}^{1}\right)\right)_{h \Sigma_{n}}\right.\right. \\
& \downarrow \cong \\
& (\bigotimes \tilde{\mathbb{K}}[(\quad)]))_{h \Sigma_{n}} \\
& \downarrow \cong \\
& \tilde{\mathbb{K}}\left[\bigwedge^{n}()_{h \Sigma_{n}}\right]
\end{aligned}
$$

and because each $\left.\left(\tilde{\mathbb{K}}\left[\left(\bigwedge^{n}\right)_{h \Sigma_{n}}\right]\right)\right)_{h \Sigma_{n}}$ is $n$-homogeneous this composite is an equivalence. If one further changes the definition of $J / J^{n+1}$ to be the mapping cone (as opposed to the strict cofiber) of the multiplication map, one can then repeat our earlier discussion, using $K^{w}=S^{w} \circ N \circ \tilde{\mathbb{K}}[] \circ U \circ \Gamma \circ U$ instead of $K$. One can let $P_{n}^{W} \mathbf{E x p}=\left(P_{n} \mathbf{E x p}\right) K^{w, *+1}$, which is a degree $n$ equivalence-preserving functor from $E_{\infty}(\mathbb{K})$ to $C h(\mathcal{K})$, and one sees that

$$
P_{n}^{W} \operatorname{Exp} \stackrel{\simeq}{\longrightarrow} I / I^{n}
$$

where $I / I^{n}=J / J^{n}\left(K^{w, *+1}\right)$.

\section{REFERENCES}

[A] G. Arone, The Mitchell-Richter filtration of loops on Stiefel manifolds stably splits, Proc. Amer. Math. Soc. 129 (2001), 1207-1211. MR 2002d:55004

[A-M] G. Arone and M. Mahowald, The Goodwillie tower of the identity functor and the unstable periodic homotopy of spheres, Invent. Math. 135 (1999), no. 3, 743-788. MR 2000e:55012 
[Be] S. Betley, Stable derived functors, the Steenrod algebra and homological algebra in the category of functors, Fund. Math. 168 (2001), no. 3, 279-293. MR 2002f:18025

[Bo] A. K. Bousfield, Homogeneous functors and their derived functors, unpublished manuscript.

[B-M] M. Basterra and R. McCarthy, Г-homology, topological André-Quillen homology and stabilization, Topology Appl. 121 (2002), no. 3, 551-566. MR 2003d:13013

[CCGH] G. Carlsson R. Cohen, T. Goodwillie, and W.-C. Hsiang, The free loop space and the algebraic $K$-theory of spaces, $K$-Theory 1 (1987), no. 1, 53-82. MR 88i:55002

[D-P] A. Dold and D. Puppe, Homologie nicht-additiver Funktoren. Anwendungen, Ann. Inst. Fourier (Grenoble) 11 (1961), 201-312. MR 27:186

[E-M1] S. Eilenberg and S. Mac Lane, Homology theories for multiplicative systems, Trans. Amer. Math. Soc. 71 (1951), 294-330. MR 13:314c

[E-M2] S. Eilenberg and S. Mac Lane, On the groups $H(\pi, n)$, II, Ann. of Math. 60 (1954), 49-139. MR 16:391a

[BKMM] A. D. Elmendorf, I. Kriz, M. A. Mandell, and S. P. May, Rings, modules, and algebras in stable homotopy theory, Math. Surveys and Monographs, Vol. 47, Amer. Math. Soc., Providence, RI, 1997.

[G1] T. Goodwillie, Calculus I: The first derivative of pseudoisotopy theory, $K$-Theory 4 (1990), 1-27. MR 92m:57027

[G2] T. Goodwillie, Calculus II: Analytic functors, K-Theory 5 (1992), 295-332. MR 93i:55015

[G3] T. Goodwillie, Calculus III: The Taylor series of a homotopy functor, in preparation.

[J-P] M. Jibladze and T. Pirashvili, Cohomology of algebraic theories, J. Algebra 137 (1991), 253-296. MR 92f:18005

[J-M1] B. Johnson and R. McCarthy, Linearization, Dold-Puppe stabilization, and Mac Lane's Q-construction, Trans. Amer. Math. Soc. 350 (1998), 1555-1593. MR 98i:18007.

[J-M2] B. Johnson and R. McCarthy, Taylor towers for functors of additive categories, J. Pure Appl. Algebra 137 (1999), 253-284. MR 2000b:18018

[J-M3] B. Johnson and R. McCarthy, A classification of degree $n$ functors, to appear in Cahiers Topologie Géom. Différentielle Catég.

[K-Mc] R. Kantorovitz and R. McCarthy, The Taylor towers for rational algebraic $K$-theory and Hochschild homology, Homology Homotopy Appl. 4 (2002), no. 1, 191-212.

[K-M] I. Kriz and J. P. May, Operads, algebras, modules and motives, Astérisque 233, Société Mathématique de France, 1995. MR 96j:18006

[MO] A. Mauer-Oats, Algebraic Goodwillie calculus and a cotriple model for the remainder, preprint.

[M1] R. McCarthy, Relative algebraic K-theory and topological cyclic homology, Acta Math. 179 (1997), no. 2, 197-222. MR 99e:19006

[M2] R. McCarthy, Dual calculus for functors to spectra, Homotopy Methods in Algebraic Topology (Boulder, CO, 1999), 183-215, Contemp. Math., 271, Amer. Math. Soc., Providence, RI, 2001. MR 2002c:18009

[M] V. Minasian, André-Quillen spectral sequence for THH, Topology Appl. 129 (2003), no. 3, 273-280.

[P] T. Pirashvili, Higher additivizations, Trudy Tbiliss. Mat. Inst. Razmadze Akad. Nauk. Gruzin. SS2 91 (1988), 44-54. (Russian) MR 90k:18012

[Q1] D. Quillen, Homotopical algebra, Lecture Notes in Mathematics, vol. 43, SpringerVerlag, Berlin, 1967. MR 36:6480

[Q2] D. Quillen, On the (co-)homology of commutative rings, Proc. Sympos. Pure Math. XVII, Amer. Math. Soc., Providence, RI, 1970, 65-87. MR 41:1722

[R] B. Richter, An Atiyah-Hirzebruch spectral sequence for topological André-Quillen homology, J. Pure Appl. Algebra 171 (2002), no. 1, 59-66. MR 2003c:13012

[S] D. Simson, Stable derived functors of the second symmetric power functor, second exterior power functor and Whitehead gamma functor, Colloq. Math. 32 (1974), 49-55. MR 50:10020

[S-T] D. Simson and A. Tyc, Connected sequences of stable derived functors and their applications, Dissertationes Math. 111 (1974), 1-71. MR 51:14030 
[We] C. Weibel, An introduction to homological algebra, Cambridge University Press, Cambridge, 1994. MR 95f:18001

[W] M. Weiss, Orthogonal Calculus, Trans. Amer. Math. Soc. 347 (1995), no. 10, 3743-3796. MR 96m:55018

Department of Mathematics, Union College, Schenectady, New York 12308

E-mail address: johnsonb@union.edu

Department of Mathematics, University of Illinois at Urbana-Champaign, 1409 W. Green St., Urbana, Illinois 61801

E-mail address: randy@math.uiuc.edu 